\title{
WestVirginiaUniversity
}

THE RESEARCH REPOSITORY @ WVU

Graduate Theses, Dissertations, and Problem Reports

2009

\section{Speech Processing Front-end in Low-power Hardware}

\author{
Brandon David Rumberg \\ West Virginia University
}

Follow this and additional works at: https://researchrepository.wvu.edu/etd

\section{Recommended Citation}

Rumberg, Brandon David, "Speech Processing Front-end in Low-power Hardware" (2009). Graduate Theses, Dissertations, and Problem Reports. 2830.

https://researchrepository.wvu.edu/etd/2830

This Thesis is protected by copyright and/or related rights. It has been brought to you by the The Research Repository @ WVU with permission from the rights-holder(s). You are free to use this Thesis in any way that is permitted by the copyright and related rights legislation that applies to your use. For other uses you must obtain permission from the rights-holder(s) directly, unless additional rights are indicated by a Creative Commons license in the record and/ or on the work itself. This Thesis has been accepted for inclusion in WVU Graduate Theses, Dissertations, and Problem Reports collection by an authorized administrator of The Research Repository @ WVU. For more information, please contact researchrepository@mail.wvu.edu. 


\title{
Speech Processing Front-end in Low-power Hardware
}

\author{
by \\ Brandon David Rumberg \\ Thesis submitted to the \\ College of Engineering and Mineral Resources \\ at West Virginia University \\ in partial fulfillment of the requirements \\ for the degree of \\ Master of Science \\ in \\ Electrical Engineering \\ David W. Graham, Ph.D., Chair \\ Xin Li, Ph.D. \\ James W. Lewis, Ph.D. \\ Lane Department of Computer Science and Electrical Engineering \\ Morgantown, West Virginia \\ 2009
}

Keywords: Analog, VLSI, integrated circuits, speech processing, low-power

Copyright 2009 Brandon David Rumberg 


\begin{abstract}
Speech Processing Front-end in Low-power Hardware

by

Brandon David Rumberg
\end{abstract}

The objective of this work is to develop analog integrated circuits to serve as low-power auditory front-ends in signal processing systems. An analog front-end can be used for featureextraction to reduce the requirements of the digital back-end, or to detect and call attention to compelling characteristics of a signal while the back-end is in sleep mode. Such a front-end should be advantageous for speech recognition, noise suppression, auditory scene analysis, hearing prostheses, biological modeling, or hardware-based event detection.

This work presents a spectral decomposition system, which consists of a bandpass filter bank with sub-band magnitude detection. The bandpass filter is low-power and each channel can be individually programmed for different quality factors and passband gains. The novel magnitude detector has a 68 decibel dynamic range, excellent tracking capability, and consumes less than a microwatt of power. The system, which was fabricated in a 0.18 micron process, consists of a 16-channel filter bank and a variety of sub-band computational elements. 


\section{Acknowledgments}

This work would not have been possible without the help of many people, who have shared their time, wisdom, and resources.

I want to thank my advisor, Dr. David Graham, for his knowledge and enthusiasm, as well as the rest of my committee, Dr. Xin Li and Dr. James Lewis, for lending their expertise on the periphery of this research area. Thanks to GTronix for providing funding and insightful feedback. I also want to acknowledge Anvesh Singireddy, who worked on the other half of this project.

Also, a big thanks to my family and friends for offering patience and distractions. 


\section{Contents}

Acknowledgments $\quad$ iii

List of Figures $\quad$ vi

List of Tables viii

1 Introduction $\quad 1$

1.1 An Analog Renaissance . . . . . . . . . . . . . . . . . . . . 2

1.1.1 Sub-threshold CMOS . . . . . . . . . . . . . . . . . . 2

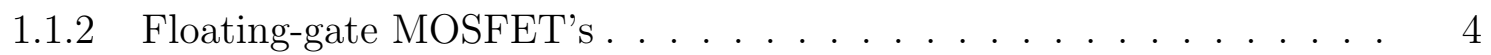

1.2 Real-time Signal Processing . . . . . . . . . . . . . . . . . . . . . . 4

1.2.1 Traditional Approach . . . . . . . . . . . . . . . . . . 4

1.2.2 Analog/Digital Synergy . . . . . . . . . . . . . . . . . 4

1.3 Overview of this work . . . . . . . . . . . . . . . 5

1.3.1 Bandpass Filter . . . . . . . . . . . . . . . . . . 6

1.3.2 Magnitude Detector . . . . . . . . . . . . . . . . . . 6

1.3.3 Audio Processing Systems . . . . . . . . . . . . . . . . . . 7

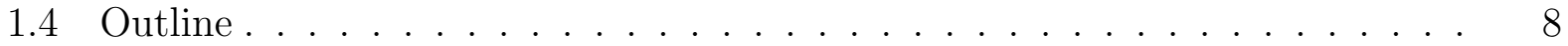

2 Bandpass Filters $\quad 9$

2.1 Continuous-time filter architectures . . . . . . . . . . . . . . . 10

2.1.1 Tow-Thomas Biquad . . . . . . . . . . . . . . . . 10

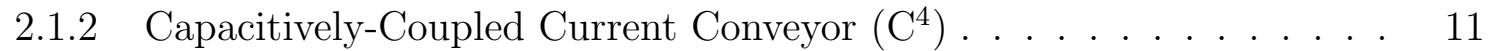

2.1.3 OTA-based $\mathrm{C}^{4} \ldots \ldots \ldots \ldots \ldots$

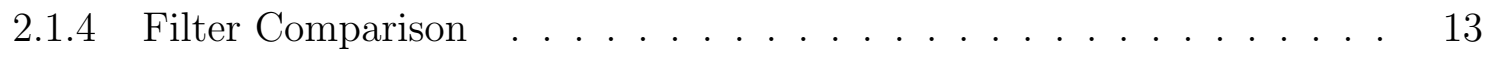

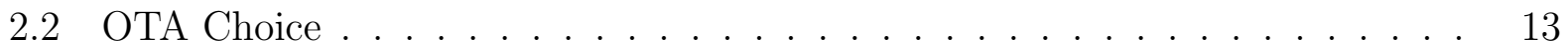

2.3 Design Procedure . . . . . . . . . . . . . . . . . . . . . 14

2.4 Ratio of Transconductances . . . . . . . . . . . . . . . . . . . . . 15

2.5 Tuning Range . . . . . . . . . . . . . . . . . . . . . . . . . . . . . . . . . . . . . . . . . . . . .

2.6 Specifications . . . . . . . . . . . . . . . . . . 17

3 Magnitude Detector $\quad 23$

3.1 Magnitude Detector Characteristics . . . . . . . . . . . . . . . . . 24

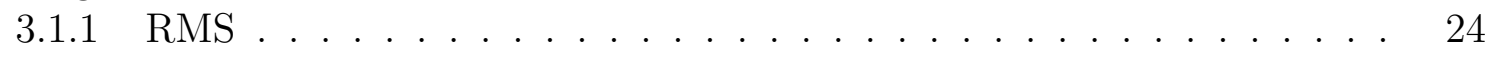

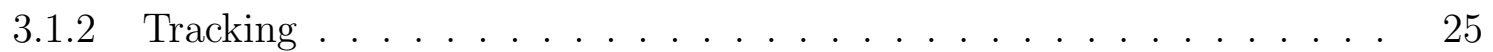


3.2 Magnitude Detector Structure . . . . . . . . . . . . . . . . 25

3.2.1 Attack and Decay . . . . . . . . . . . . . . . . . . . 25

3.2.2 Classic Diode Detector . . . . . . . . . . . . . . . . . 28

3.2.3 $G_{m}-C$ Magnitude Detectors . . . . . . . . . . . . . . . 30

3.3 Adaptive Decay Peak Detector . . . . . . . . . . . . . . . . . . . . 30

3.4 Nonlinear Filter . . . . . . . . . . . . . . . . . . . . . . . 32

3.4.1 Nonlinear Transconductor . . . . . . . . . . . . . . . . . 33

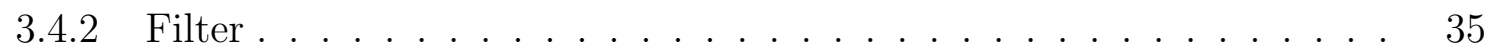

3.5 Full Magnitude Circuit . . . . . . . . . . . . . . . . . . . . . 37

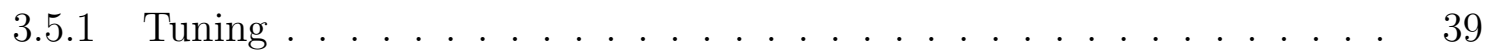

4 Analog Frequency Decomposition 43

4.1 Event-detection Filter bank . . . . . . . . . . . . . . . . . . . . 43

$4.20 .18 \mu m$ Filter bank . . . . . . . . . . . . . . . . . . . 46

5 Lateral Coupling in Cochlear Models 51

5.1 Biological Motivation . . . . . . . . . . . . . . . . . . 52

5.2 Silicon Cochlea Models . . . . . . . . . . . . . . . . . . . . 53

5.3 Lateral Coupling . . . . . . . . . . . . . . . . . . . 55

6 Low-power Hardware-based Event Detection $\quad 59$

6.1 Sound Threshold Event Detection . . . . . . . . . . . . . . . . . 60

6.2 Sound Event Classification . . . . . . . . . . . . . . . . . . . . . 62

6.3 Potential directions . . . . . . . . . . . . . . . . . . . 62

7 Conclusion / Future Directions $\quad 65$

A Alternate Magnitude Circuits $\quad 66$

A.1 Asymmetric-OTA Peak Detector . . . . . . . . . . . . . . 66

A.2 Full-Wave Peak Detector . . . . . . . . . . . . . . . . . 70

A.3 Peak Hold Detector . . . . . . . . . . . . . . . . . 70

B $C^{4}$ Derivations $\quad 74$

B.1 Derivations for an OTA based $\mathrm{C}^{4} \ldots \ldots \ldots \ldots$

B.1.1 Transfer Function . . . . . . . . . . . . . . . . . . . . . . 75

B.1.2 $\mathrm{C}^{4}$ at High Frequencies . . . . . . . . . . . . . . . . . . . 77

B.1.3 C $\mathrm{C}^{4}$ at Low Frequencies . . . . . . . . . . . . . . . . 78

B.1.4 Capacitive Feedthrough . . . . . . . . . . . . . . . 78

B.1.5 Solving for $Q_{\max } \ldots \ldots \ldots \ldots$. . . . . . . . . . . . . . . . . . . . . . . . . . . . 79

B.2 OTA-C ${ }^{4}$ Noise Analysis . . . . . . . . . . . . . . . . . . . 81

B.2.1 Noise Transfer Function for $G_{m 1}$ Noise Source . . . . . . . . . . . . . 82

B.2.2 Noise Transfer Function for $G_{m 2}$ Noise Source . . . . . . . . . . . . . 83

B.2.3 Integrated Noise . . . . . . . . . . . . . . . . . . . . 83

B.3 Power Analysis . . . . . . . . . . . . . . . . . . . . . 87

B.4 Design Procedure . . . . . . . . . . . . . . . . 87

$\begin{array}{lr}\text { References } & 90\end{array}$ 


\section{List of Figures}

1.1 Transistor symbols. . . . . . . . . . . . . . . . . . . . . 3

1.2 Example of a system with an analog front-end and a digital back-end. . . . . 5

1.3 Analog front-end for real-time signal processing systems. . . . . . . . . . . 6

1.4 Frequency decomposition with an analog front-end. . . . . . . . . . . . 7

2.1 Tow-Thomas biquad in a bandpass configuration. . . . . . . . . . . . 10

2.2 Transistor based $\mathrm{C}^{4} \ldots \ldots \ldots \ldots$

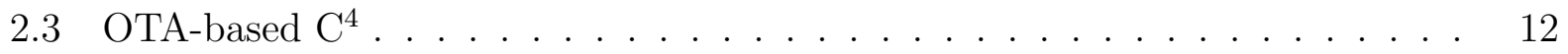

2.4 Symmetric-bump OTA . . . . . . . . . . . . . . . . . . . . . . . . . . 14

2.5 Effect of $G_{m 1} / G_{m 2}$ on different $\mathrm{C}^{4}$ characteristics $\ldots \ldots \ldots$

$2.6 \mathrm{C}^{4}$ THD for different transconductance ratios . . . . . . . . . . . . . 18

2.7 Emulating a 32 channel filter bank by a linear stepping of bias voltages. . . . 19

$2.8 \mathrm{C}^{4}$ biased from $5 \mathrm{~Hz}$ to $50 \mathrm{kHz}$ with a gain of (a) $0 \mathrm{~dB}$ (b) $4 d \mathrm{~dB} \ldots \ldots$

2.9 Four sub-band characterizations of the $\mathrm{C}^{4} \ldots \ldots \ldots \ldots 21$

$2.10 \mathrm{C}^{4}$ linearity. . . . . . . . . . . . . . . . . . . . . . 22

3.1 Block diagram of magnitude detector . . . . . . . . . . . . . . . . 24

3.2 Effect of integration window on RMS computation . . . . . . . . . . . 26

3.3 General peak detector architecture . . . . . . . . . . . . . . . . 27

3.4 Classic diode detector . . . . . . . . . . . . . . . . . . . . . . . . . . 28

3.5 Constant-decay peak detector . . . . . . . . . . . . . . . . . . . . . . . . . 29

3.6 Adaptive-decay peak detector . . . . . . . . . . . . . . . . . . . 31

3.7 Comparing the constant decay and adaptive decay peak detectors . . . . . . 32

3.8 Need for a nonlinear filter . . . . . . . . . . . . . . . . . . . 33

3.9 Nonlinear OTA . . . . . . . . . . . . . . . . . . . . . . . . . . . 34

3.10 Nonlinear lowpass filter . . . . . . . . . . . . . . . . . . . . . . . 35

3.11 AC response of the nonlinear filter . . . . . . . . . . . . . . . . 36

3.12 Step response of the nonlinear filter . . . . . . . . . . . . . . . . 37

3.13 Proposed magnitude detector . . . . . . . . . . . . . . . . 38

3.14 Dynamic range of the magnitude circuit $\ldots \ldots \ldots$. . . . . . . . . 39

3.15 Output referred noise for the magnitude circuit . . . . . . . . . . . . 40

3.16 Magnitude circuit tracking the RMS of an unfiltered speech segment . . . . . 41

3.17 Tuning algorithm for the magnitude circuit . . . . . . . . . . . . . . 42

4.1 Event-detection filter bank . . . . . . . . . . . . . . . . . . . . . . 44 
4.2 Step response of an analog filter bank . . . . . . . . . . . . . . . . . 45

4.3 Decomposition of two tones with an analog filter bank . . . . . . . . . . 46

4.4 AC response of the $0.18 \mu m$ filter bank . . . . . . . . . . . . . . . . . . . . . 47

4.5 Speech spectrogram with analog filter bank . . . . . . . . . . . . . 48

4.6 Speech sub-banding with analog filter bank . . . . . . . . . . . . . . . . . 49

4.7 Layout of the $0.18 \mu m$ auditory front-end . . . . . . . . . . . . . . . 50

5.1 Lateral coupling concept . . . . . . . . . . . . . . . . . . . 52

5.2 Lateral coupling with a cascade of bandpass resonators . . . . . . . . . . . 53

5.3 Lateral coupling of higher-frequency filters with "dummy" filters . . . . . . . 55

5.4 Schematic for a lateral-coupling filter tap . . . . . . . . . . . . . . . . . 57

5.5 AC characteristics of lateral coupling filter . . . . . . . . . . . . . . 58

6.1 Idea for hardware detection of sound events. . . . . . . . . . . . . 60

6.2 Block diagram for the fabricated event detection system. . . . . . . . . . 61

6.3 Die shot of the event detection chip . . . . . . . . . . . . . . . 64

A.1 Asymmetric OTA . . . . . . . . . . . . . . . . . 67

A.2 Asymmetric-OTA peak detector . . . . . . . . . . . . . . . . 68

A.3 Floating-gate asymmetric-OTA peak detector operation. . . . . . . . . . 69

A.4 Full-wave peak detector . . . . . . . . . . . . . . . . . . 71

A.5 Peak-hold detector . . . . . . . . . . . . . . . . . . . . . . . . . . . . . . . . . . . . . . . . . . . . . 73

A.6 Peak-hold detector operation . . . . . . . . . . . . . . . . 73

B.1 Schematic of the OTA-C $\mathrm{C}^{4}$ for transfer-function derivation. . . . . . . . . . . 74

B.2 Schematic of the OTA-C ${ }^{4}$ for transfer-function derivation at high frequencies. 76

B.3 Schematic of the OTA-C ${ }^{4}$ for transfer-function derivation at low frequencies. 78

B.4 Schematic of the OTA- $\mathrm{C}^{4}$ for noise analysis. . . . . . . . . . . . . . 82 


\section{List of Tables}

2.1 Comparison of $G_{m}-C$ filters . . . . . . . . . . . . . . . . 13

$2.20 .18 \mu m \mathrm{C}^{4}$ characteristics $\ldots \ldots \ldots \ldots \ldots$ 


\section{Chapter 1}

\section{Introduction}

Development of cheap and tiny sensors enables electronics to have many windows on the world. Integration of a myriad of sensors presents new challenges for real-time signal processing, particularly if the device is battery powered. A flood of information from the sensors must be processed concurrently in order to perceive the environment. Present techniques are not adequate for low-power perception tasks.

Fortunately, efficient perception systems are not unprecedented, and for inspiration we need look no further than our own sensory systems. Natural signal processing handles a wealth of simultaneous signals, operates with modest power consumption, and is robust in the presence of faulty components and noisy input. The computational elements are nonlinear and non-ideal, but complex operations are performed efficiently by exploiting the complete physical characteristics of the elements, rather than restricting their operation to linear regions or abstracting them as having discrete states [1]. Low-level processing extracts important features to represent the environment abstractly with reduced dimensionality, this decreases the burden on higher-level processing.

The objective of this work is to develop analog integrated circuits to serve as lowpower front-ends in signal processing systems. An analog front-end can be used for featureextraction to allow a digital back-end to operate on the information at a higher abstraction level, or to detect and call attention to compelling characteristics of a signal. 


\subsection{An Analog Renaissance}

In [2], analog computation is defined as "the use of one physical system as a model for another system, more difficult to construct or measure, that obeys equations of the same form." An example is a network of electrical components that is described by the same differential equations as a mass and spring system. In modern parlance, analog means continuous-valued, and a circuit is said to be analog if it processes continuous-valued signals. ${ }^{1}$ Continuous signals are important because nearly all sensors transduce continuous signals, and the physical world deals with continuous signals.

Some common analog circuits are amplifiers, filters, and oscillators. Like the mass-spring analogy, these circuits are designed by connecting circuit elements such that the equations describing them are the functions they are intended to perform. Given that the voltage-tocurrent relationship for a circuit element may be linear, differential, exponential, quadratic, etc., we can imagine that very complex functions can be synthesized with a small number of circuit elements.

Recently, researchers have been developing a new portfolio of analog computational elements. Many of these circuits are biologically inspired [3], biased in sub-threshold for extremely low-power consumption [4], and achieve an unprecedented degree of programmability (for analog circuits) by using floating-gate transistors. Systems built with these circuits are able to exploit the parallel and localized nature of many algorithms, such as sub-band or neural network processing.

\subsubsection{Sub-threshold CMOS}

While analog circuits traditionally used BJT's (bipolar junction transistors) due to their high transconductance efficiency and gain, the vast majority of recent work on analog integrated circuits has focused on MOSFET's (metal-oxide-semiconductor field-effect transistors). CMOS (complementary MOS) fabrication processes have become ubiquitous for digital integrated circuits, and are thus the cheapest option for integrated circuit fabrication. By implementing analog circuits in CMOS processes, both the analog and digital portions of a

\footnotetext{
${ }^{1}$ Although the signal is continuous-valued it has finite resolution due to noise generated by the circuit.
} 


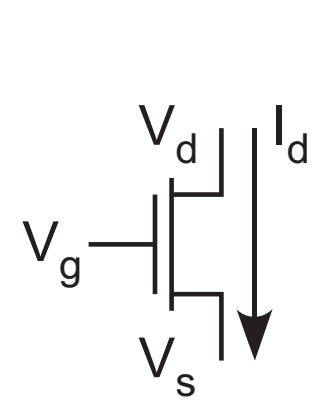

(a)

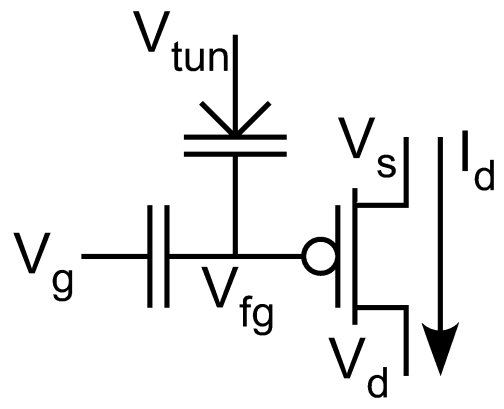

(b)

Figure 1.1: (a) Symbol for an n-channel MOSFET. (b) Schematic of a p-channel floating-gate transistor

system can be integrated on the same die, reducing cost and size. Even higher levels of integration can be attained since many sensors (e.g., imagers and thermometers) can be made in standard CMOS processes, and many other sensors (e.g., microphones and accelerometers) can be included with additional MEMS (microelectromechanical systems) fabrication steps.

Low-power CMOS analog circuits can be made by biasing the transistors in the weakinversion region (sub-threshold). In sub-threshold, the current through an n-channel MOSFET (fig. 1.1(a)) is described by

$$
I=I_{0} e^{\frac{\kappa V_{g}}{U_{T}}}\left(e^{-\frac{V_{s}}{U_{T}}}-e^{-\frac{V_{d}}{U_{T}}}\right)
$$

where $\kappa$ is the subthreshold slope factor, $I_{0}$ depends on process constants and the dimensions of the transistor, and $U_{T}$ is the thermal voltage given by $U_{T}=k T / q \approx 26 m V[3,4]{ }^{2}$ Drain current is due to diffusion and is governed by the Boltzmann distribution, giving credence to the use of sub-threshold MOSFET's for modeling neural computations, which operate under similar physical principles. Transistors in sub-threshold typically have currents below $1 \mu A$ and reliably operate with currents in the $p A$ range and lower. The exponential voltagecurrent relation (similar to BJT's) can be used to implement compressive/expansive functions and makes it easier to build constant relative bandwidth filter banks. By operating in subthreshold, complex and useful systems can be built which consume less than a $\mu W$.

\footnotetext{
${ }^{2} k$ is Boltzmann's constant, $T$ is temperature in kelvin, and $q$ is the charge of an electron.
} 


\subsubsection{Floating-gate MOSFET's}

Floating-gate transistors (fig. 1.1(b)) are important for creating programmable analog circuits. In a floating-gate transistor, the floating-gate $\left(V_{f g}\right)$ is completely surrounded by insulator, and will thus hold a constant charge. The charge on the floating-gate, along with the coupling of node $V_{g}$ (and to a lesser extent, all other nodes), sets the current flowing through the transistor. Through electron tunneling and hot electron injection, charge on the floating-gate can be increased and decreased [5]. Floating-gates can be used to make precise and programmable current sources [6], to tune out mismatch resulting from fabrication tolerances [7], or they can be used as memory elements in adaptive systems $[8,9]$.

\subsection{Real-time Signal Processing}

\subsubsection{Traditional Approach}

A typical real-time signal processing system has at least one sensor (e.g., microphone, accelerometer, photodiode, thermistor, or piezo) which outputs a time-varying voltage or current. This signal then goes through some signal conditioning before it can be digitized. This conditioning likely includes an amplifier to increase the signal to fill the dynamic range of the analog-to-digital converter, and an anti-aliasing filter to bandlimit the signal to the highest frequency of interest. It may also include filtering to remove line noise or some type of buffer to isolate the sensor from the rest of the system. After conditioning, the signal is measured with an analog-to-digital converter which will produce a stream of numbers for a microprocessor, DSP, or FPGA (field-programmable gate array). DSP (digital signal processing) allows the developer to program commodity hardware at a high abstraction level. Compared to analog circuit design, development time for DSP is shorter and changes can be made more easily.

\subsubsection{Analog/Digital Synergy}

Since the output of the sensors is inherently analog, it makes sense to ask if analog preprocessing can offload some of the DSP burden. In a system like fig. 1.2, common processing 


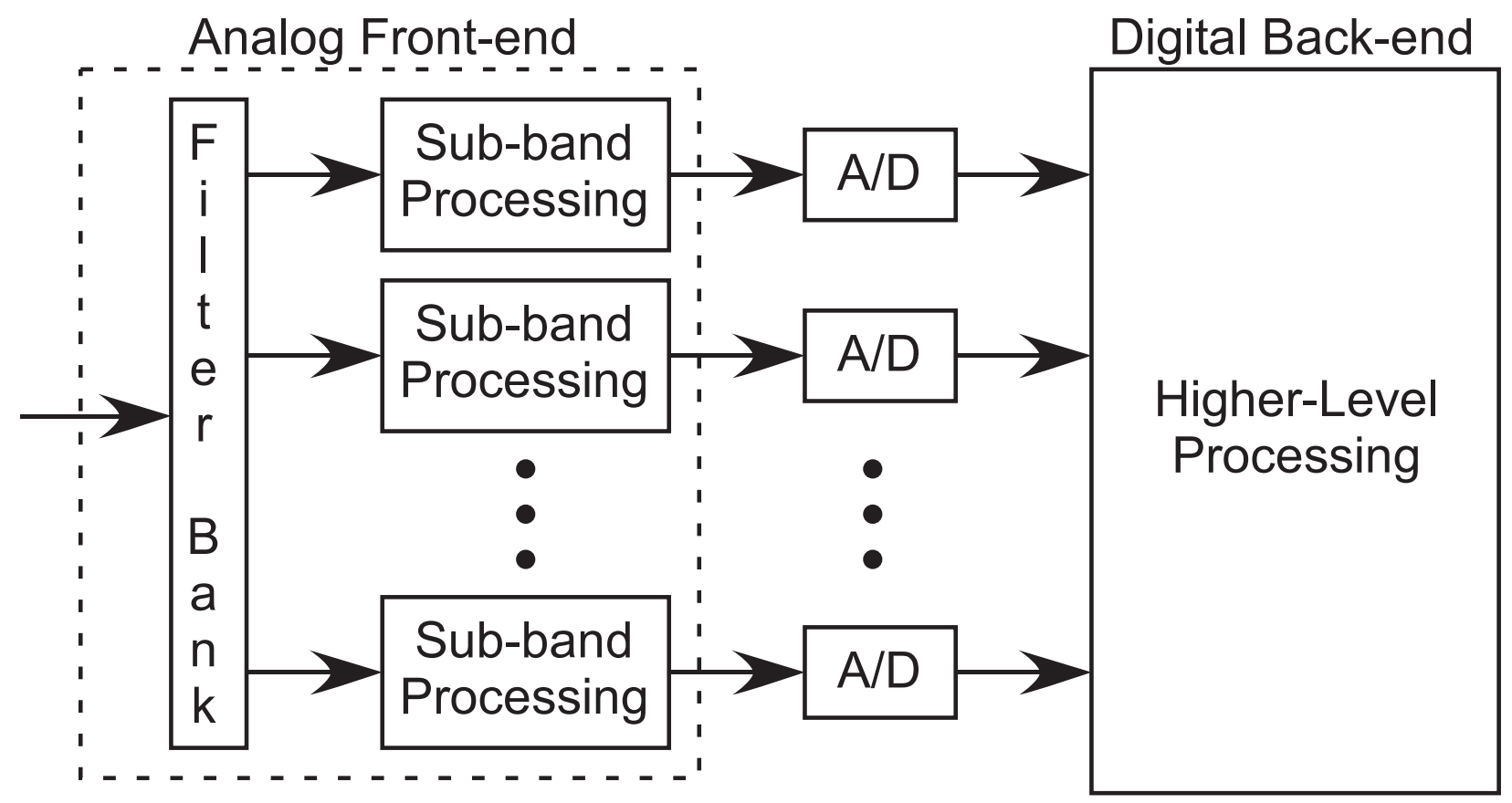

Figure 1.2: Example of a system with an analog front-end and a digital back-end.

tasks can be performed in the analog front-end, while higher level processing is performed in the digital back-end. This achieves a compromise between efficiency and development time [10]. The DSP starts with less redundant data, which reduces the processing load and simplifies development.

In this work, we have developed an analog front-end for audio processing systems which is illustrated in figure 1.3. This system decomposes the frequencies with a bank of bandpass filters. Following the filter bank are sub-band processing circuits which can be connected in various configurations.

\subsection{Overview of this work}

This document details my work on two building blocks for audio-frequency spectral decomposition, a bandpass filter and a magnitude detector. The design objectives are lowpower (tens of $\mu W^{\prime}$ 's for entire system), high-performance (10 bits), and programmability. 


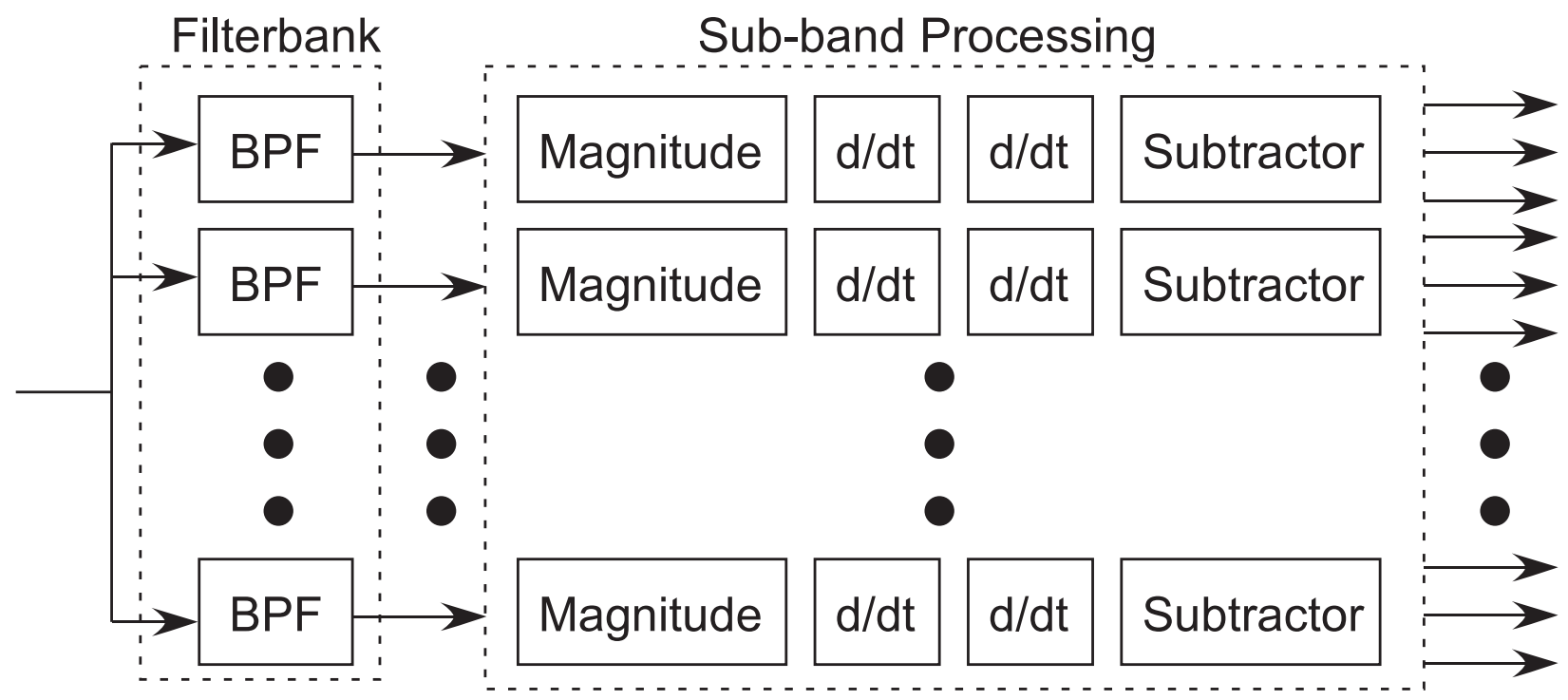

Figure 1.3: Analog front-end fabricated in a $0.18 \mu \mathrm{m}$ process. The sub-band blocks can be connected in different combinations.

\subsubsection{Bandpass Filter}

A bandpass filter has been designed which has approximately $60 \mathrm{~dB}$ of dynamic range, consumes less than a $\mu W$ of power at speech frequencies, and can be programmed for different gains, center frequencies, and bandwidths. This filter has been arrayed to construct programmable, constant relative bandwidth (i.e. constant-Q) filter banks. The filter is a modification of the capacitively-coupled current conveyor architecture.

\subsubsection{Magnitude Detector}

A novel magnitude detector has been developed which has $68 d B$ of dynamic range, excellent tracking capability, and consumes less than a $\mu W$ of power. For this circuit, we developed a nonlinear low-pass filter to smooth small ripples while being responsive to large amplitude swings. This magnitude detector has been used for spectral power detection in conjunction with filter banks. 

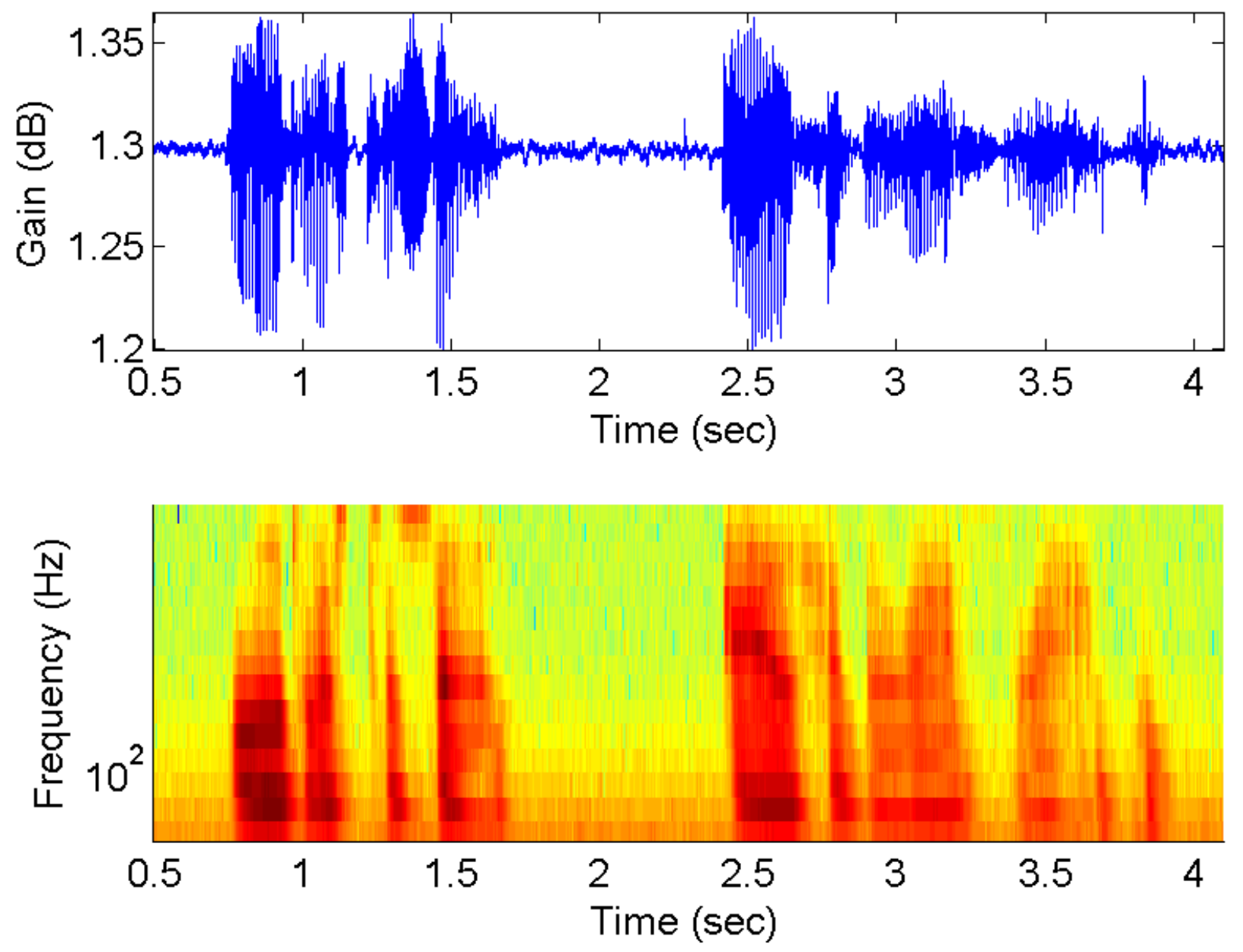

Figure 1.4: Spectrogram of a speech signal generated by an analog filter bank with a magnitude detection circuit in each sub-band.

\subsubsection{Audio Processing Systems}

We have constructed several analog front-ends, each consisting of an array of bandpass filters with a magnitude detector in each sub-band. Each implementation has included an assortment of sub-band processing blocks which process the sub-banded signal or the power of the sub-banded signal. The first system (fig. 1.3) was fabricated on a $0.18 \mu m$ process, has 16 channels, and the sub-band processing blocks consist of magnitude detection, differentiation, and subtraction. These sub-band blocks can be connected in different combinations, and all biases are set by floating-gate transistors. Figure 1.4 shows a spectrogram generated from the sub-banded magnitude detector outputs. A filter bank with reduced performance was 
included as part of a field-programmable analog array (FPAA) on a $0.35 \mu \mathrm{m}$ process. The sub-band blocks can be synthesized from reconfigurable circuits, making the system useful for prototyping. Another filter bank with 8 channels was used as the front-end for a hardwarebased event detection system on a $0.5 \mu \mathrm{m}$ process.

In future work, these systems may be applied in the context of auditory scene analysis, speech recognition, noise suppression, hearing prostheses, biological modeling, or hardwarebased event detection.

\subsection{Outline}

In the next chapter, the design of the bandpass filter is detailed. This includes the choice of the $\mathrm{C}^{4}$ filter architecture, a design procedure for an OTA (operational transconductance amplifier) based $\mathrm{C}^{4}$, and the results of the design. Chapter 3 presents our new magnitude detector along with the nonlinear filter used in that circuit. Chapter 4 deals with systems created from the combination of bandpass filter banks and magnitude detectors. In chapter 5 , we discuss the use of the $\mathrm{C}^{4}$ filter in a cochlear model which mimics the effect of lateral coupling along the basilar membrane. Chapter 6 describes the use of analog audio front-ends for low-power hardware-based event detection, and chapter 7 concludes the document. 


\section{Chapter 2}

\section{Bandpass Filters}

An important step in many processing algorithms is spectral decomposition. Frequency decomposition can be implemented efficiently in analog integrated circuits with electrical resonators. For this project a low-power continuous-time band-pass filter with approximately $60 \mathrm{~dB}$ of dynamic range has been developed. The filter architecture is the capacitively-coupled current conveyor, with the transistors replaced by OTA's. It has a capacitively-coupled input and the output DC level can be set independent of the transfer function, making the filter easy to use. The ability to tune the gain and quality factor post-fabrication make the filter ideal for programmable filter banks.

A filter's performance is measured by its dynamic range. For this project the dynamic range was defined as the ratio of the largest signal with tolerable distortion (for this project, $1 \%$ THD (total harmonic distortion)), to the noise generated by the circuit (which sets the minimum detectable signal). There is a trade-off between dynamic range, and size and power [11]. In our programmable filter bank each filter is identical, therefore they must meet the dynamic range when biased anywhere in the auditory spectrum. This three decade frequency range makes it more difficult to optimize for size and power consumption.

All data in this chapter are from a filter fabricated in a $0.18 \mu \mathrm{m}$ process. 


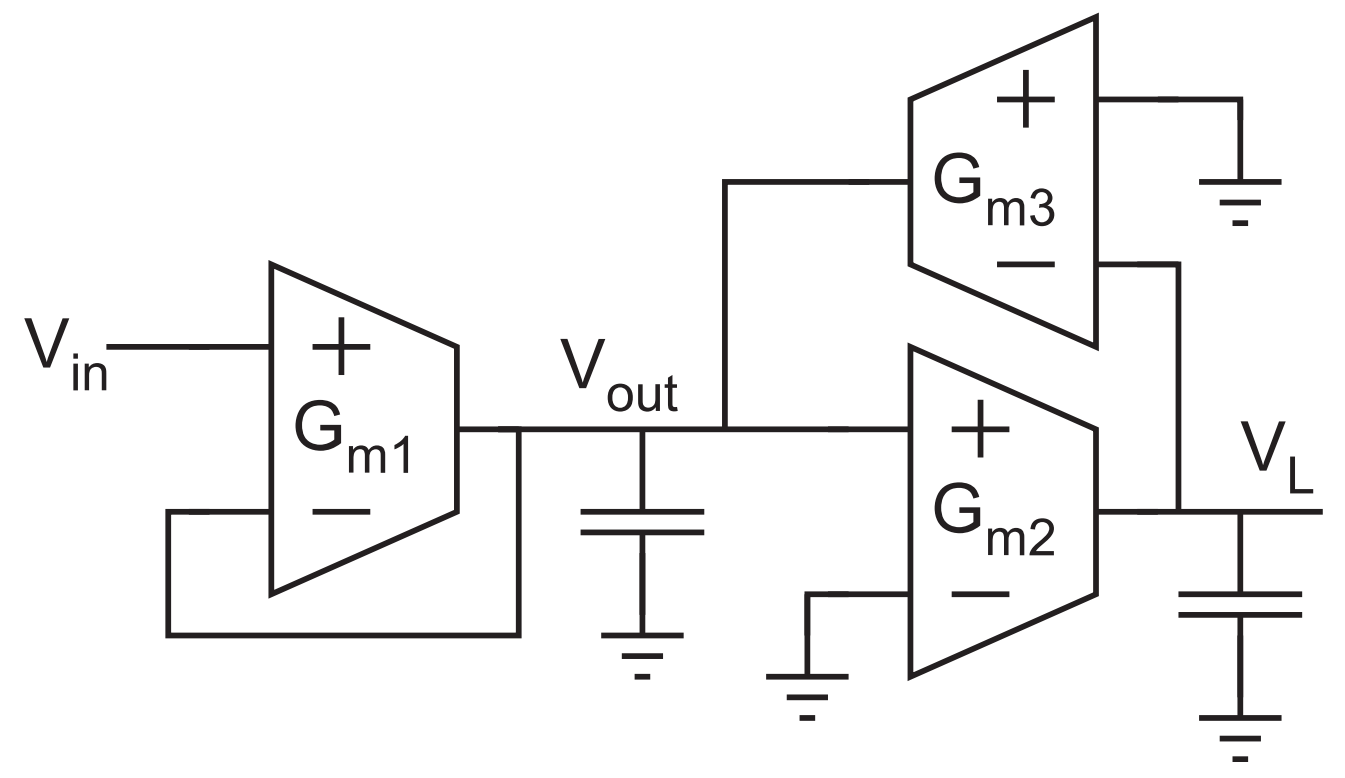

Figure 2.1: Tow-Thomas biquad in a bandpass configuration.

\subsection{Continuous-time filter architectures}

Continuous-time CMOS filters come in the form of transconductor capacitor $\left(G_{m}-C\right)$ and log-domain (or root-domain for above-threshold). Log-domain filters are current-mode and thus not appropriate for our voltage-mode system. The only options that exist for $G_{m^{-}}$ $C$ bandpass filters, that can produce complex poles (i.e. resonance), are the Tow-Thomas biquad and the capacitively-coupled current conveyor. These two varieties of $G_{m}-C$ filters are considered in the following sections.

\subsubsection{Tow-Thomas Biquad}

The Tow-Thomas is the most common $G_{m}-C$ filter. For the bandpass form of the TowThomas, the transfer function is

$$
\frac{V_{\text {out }}}{V_{\text {in }}}=\frac{s \frac{G_{m 1} C_{2}}{G_{m_{2}} G_{m 3}}}{s^{2} \frac{C_{1} C_{2}}{G_{m 2} G_{m 3}}+s \frac{G_{m 1} C_{2}}{G_{m 2} G_{m 3}}+1}
$$

The center frequency is set by $G_{m 2}$ and $G_{m 3}$ and is given by

$$
f_{c}=\sqrt{\frac{C_{1} C_{2}}{G_{m 2} G_{m 3}}}
$$




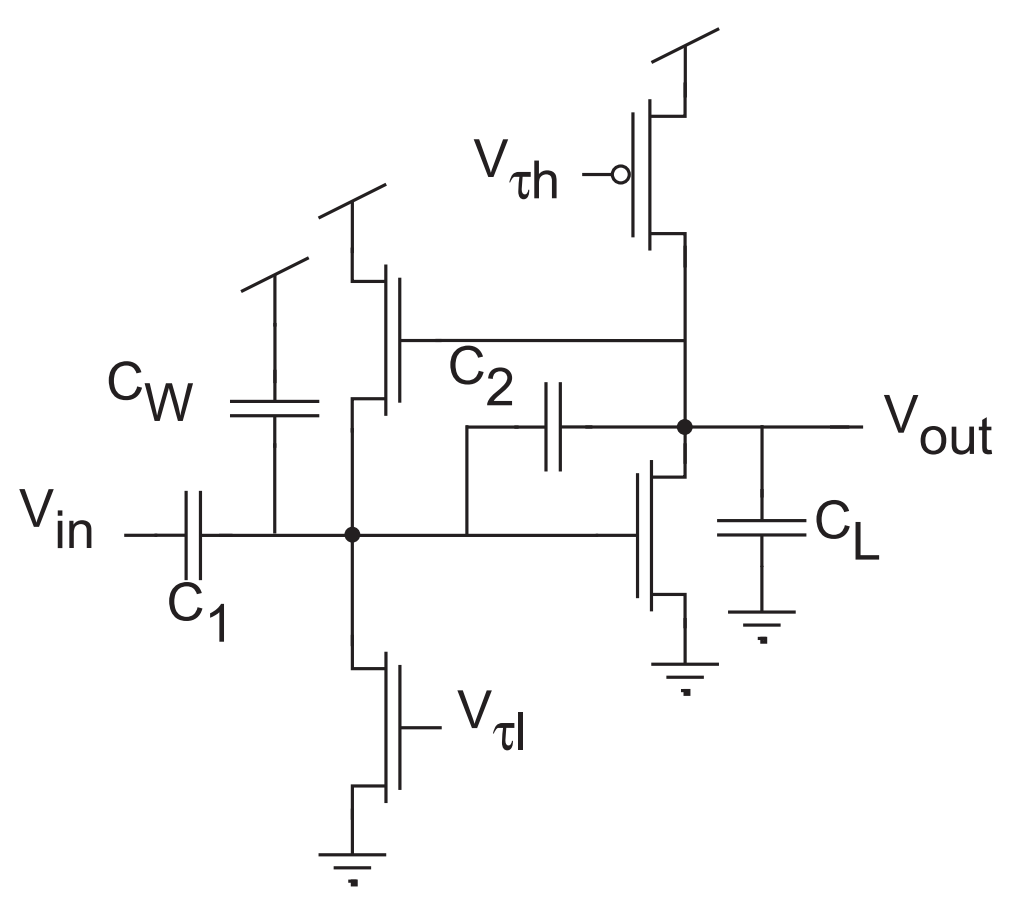

Figure 2.2: Transistor based $\mathrm{C}^{4}$.

After setting the center frequency, the Q (quality factor $=f_{c} /$ bandwidth) is set with $G_{m 1}$.

$$
Q=\sqrt{\frac{C_{1} G_{m 2} G_{m 3}}{C_{2}}} \frac{1}{G_{m 1}}
$$

\subsubsection{Capacitively-Coupled Current Conveyor $\left(\mathrm{C}^{4}\right)$}

The $\mathrm{C}^{4}$ (Fig. 2.2) is a compact, low-power, bandpass filter. Each corner frequency can be individually tuned. $V_{\tau l}$ controls the low corner frequency and $V_{\tau h}$ controls the high corner frequency. Programmability can be achieved with the $\mathrm{C}^{4}$ by use of floating gate transistors to set the bias currents $I_{\tau l}$ and $I_{\tau h}[12]$.

The transfer function for the $\mathrm{C}^{4}$ is

$$
\frac{V_{\text {out }}}{V_{\text {in }}}=-\frac{C_{1}}{C_{2}} \frac{s \tau_{l}\left(1-s \tau_{f}\right)}{1+s\left(\tau_{l}+\tau_{f}\left(\frac{C_{O}}{C_{2}}-1\right)\right)+s^{2} \tau_{h} \tau_{l}}
$$

where the time constants are given by

$$
\tau_{h}=\frac{C_{T} C_{O}-C_{2}^{2}}{C_{2} g_{m 4}} \quad \tau_{l}=\frac{C_{2}}{g_{m 1}} \quad \tau_{f}=\frac{C_{2}}{g_{m 4}}
$$




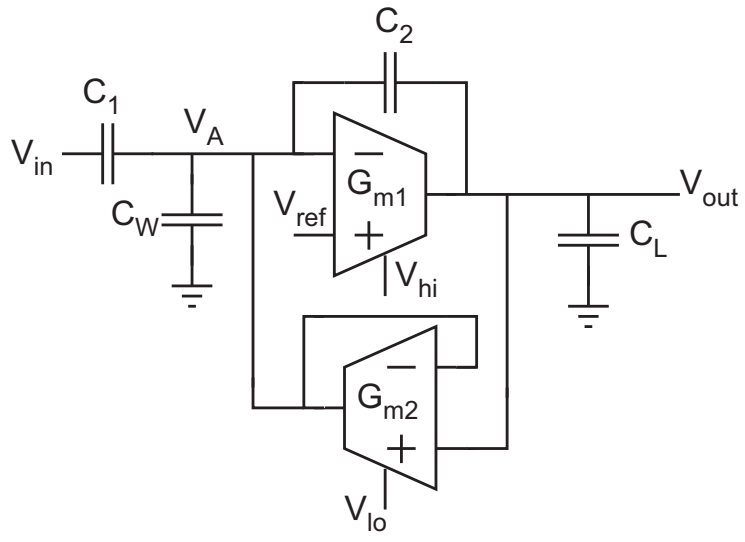

(a)

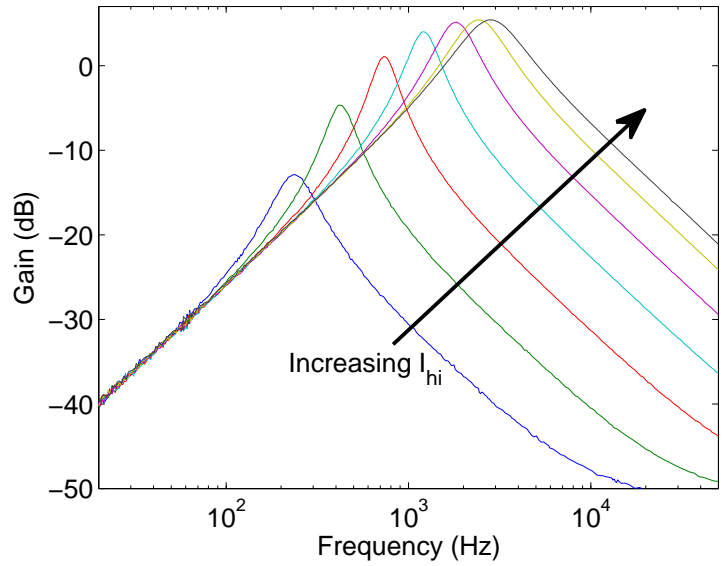

(b)

Figure 2.3: (a) OTA-based $\mathrm{C}^{4}$ (b) Sweeping $G_{m 1}$ while $G_{m 2}$ is held constant. The gain increases as $G_{m 1}$ increases. The $\mathrm{Q}$ increases until it reaches $Q_{\max }$ and then decreases.

and where the total capacitance is given by $C_{T}=C_{1}+C_{2}+C_{W}$, and the output capacitance is given by $C_{O}=C_{2}+C_{L}$. The feedthrough time constant given by $\tau_{f}$ typically has little effect on the circuit in the region near the passband.

\subsubsection{OTA-based $\mathrm{C}^{4}$}

The operating point for the transistor-based $\mathrm{C}^{4}$ is set by the source follower feedback from the output node. The offset of the source follower $\left(V_{\text {out }}-V_{A}\right)$ depends on the time constants, this causes each sub-band in a filter bank to have a different DC level. Even worse, the offset causes the operating point of the common source amplifier to be shifted from the center of its linear range, causing increased distortion. To overcome this problem, we replaced the common source amplifier with an inverting gain OTA (operational transconductance amplifier), and replaced the source follower with a follower configured OTA, resulting in the OTA-based $\mathrm{C}^{4}$ (fig. 2.3). In this configuration the filter operates in the center of the linear range, and the DC level for an entire filter array can be set by $V_{\text {ref }}$. No previous implementations of an OTA-based $\mathrm{C}^{4}$ have been reported in the literature.

The corner frequencies are orthogonally tunable through $G_{m 1}$ and $G_{m 2}$. The transfer 
Table 2.1: Comparison of $G_{m}-C$ filters

\begin{tabular}{cc}
$\mathrm{C}^{4}$ & Tow-Thomas \\
\hline 2 OTAs & $3-4$ OTAs \\
Corners orthogonally tunable & Corners not orthogonally tunable \\
4 capacitors & 2 capacitors \\
AC coupled input & Not AC coupled (Q is DC dependent) \\
Variable gain (tunable post-fab) & Fixed at unity gain
\end{tabular}

function for the OTA-based $\mathrm{C}^{4}$ is

$$
\frac{V_{\text {out }}}{V_{\text {in }}}=\frac{s \frac{C_{1}}{G_{m 2}}\left(s \frac{C_{2}}{G_{m 1}}-1\right)}{s^{2} \frac{C_{O} C_{T}-C_{2}{ }^{2}}{G_{m 1} G_{m 2}}+s\left(\frac{C_{L}}{G_{m 1}}+\frac{C_{2}}{G_{m 2}}\right)+1}
$$

were $C_{T}=C_{1}+C_{2}+C_{W}$ and $C_{O}=C_{2}+C_{L}$. The capacitor values set the maximum $\mathrm{Q}$ and the maximum gain. Since the gain and $\mathrm{Q}$ are both dependent on the transconductances, they can be tuned post-fabrication. Derivations for the OTA-based $\mathrm{C}^{4}$ are included in appendix B.

\subsubsection{Filter Comparison}

Both the Tow-Thomas and the $\mathrm{C}^{4}$ have similar dynamic range for the same die area. Table 2.1 compares the characteristics of the filters. The $\mathrm{C}^{4}$ architecture was chosen for all systems discussed in this document, due to its ease of use and programmable gain. It is easy to use because of the AC-coupled input and because the output DC level for a filter array can be set globally with one reference voltage. The $\mathrm{C}^{4}$ is also more customizable as the maximum pass-band gain can be arbitrarily set by the capacitor ratio $C_{1} / C_{2}$ and the gain can be tuned post-fabrication to any value less than the maximum gain.

\subsection{OTA Choice}

For our implementation of the OTA-based $\mathrm{C}^{4}$, we used an OTA linearized with symmetric bump elements (fig. 2.4) [13]. The bump transistors steal current from the input pair, making the tanh curve more linear. This is done without adding noise, as the noise from the bump transistors gets canceled out at the output [14]. 


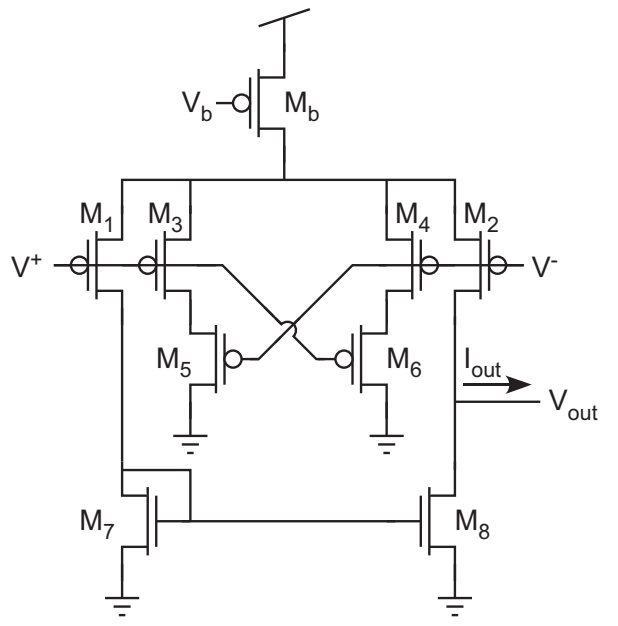

(a)

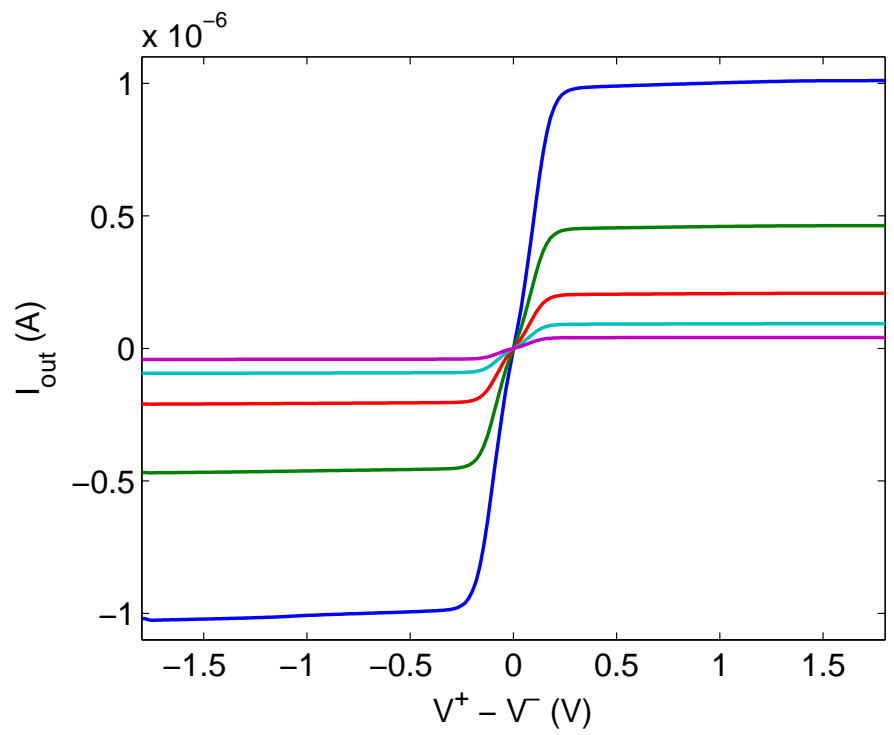

(b)

Figure 2.4: (a) Schematic of symmetric-bump OTA used in the $\mathrm{C}^{4}$ (b) I-V curves for the symmetric-bump OTA.

\subsection{Design Procedure}

Here we present a method for designing an OTA- ${ }^{4}$ for a given combination of quality factor, SNR (signal-to-noise ratio), center frequency, and $V_{i n, \max }$.

1. Design $C_{L}$ sufficiently such that $C_{L} \gg C_{T}, C_{T}$ will be determined later.

2. Choose $C_{2}$ to meet given SNR specs. This is assuming $V_{\text {out }}=V_{L}$.

$$
\begin{aligned}
S N R & =10 \log _{10}\left(\frac{4 V_{L} C_{2}}{N q}\right) \\
C_{2} & =\frac{N q}{4 V_{L}} 10^{\frac{S N R}{10}}
\end{aligned}
$$

(where $N$ is the number of noise elements in the OTA and $V_{L}$ is the linear range of the OTA)

3. If the passband gain is given, calculate $C_{1}$.

$$
\begin{aligned}
& A_{v}=\frac{-C_{1}}{2 C_{2}} \\
& C_{1}=2 C_{2} A_{v}
\end{aligned}
$$


4. Choose $C_{T}$ for linearity.

$$
\begin{aligned}
V_{\text {out }, \text { max }} & =A_{v} V_{\text {in, } \text { max }}=\frac{C_{1}}{2 C_{2}} V_{\text {in, } \text { max }}=\frac{C_{T}}{2 C_{2}} V_{L} \\
& \approx 2 Q_{\text {max }}^{2} V_{L} \\
C_{T} & \geq \frac{C_{1} V_{i n, \text { max }}}{V_{L}} \\
C_{T} & =4 C_{2} Q_{\text {max }}
\end{aligned}
$$

5. If $A_{v}$ is not given.

$$
\begin{aligned}
V_{i n, \max } & \approx \frac{2 C_{2}}{C_{1}} V_{L} \\
C_{1} & =\frac{2 C_{2} V_{L}}{V_{i n, \max }}
\end{aligned}
$$

6. Calculate the transconductances for the desired time constants

$$
\begin{gathered}
4 \pi Q_{\max } C_{2} f_{\text {center }}=4 \pi \frac{1}{2} \sqrt{C_{T}} C_{2} C_{2} \frac{1}{2 \pi} \sqrt{\frac{G_{m 2}^{2} \frac{C_{L}}{C_{2}}}{C_{T} C_{O}}}=G_{m 2} \\
G_{m 2}=4 \pi Q_{\max } C_{2} f_{\text {center }} \\
G_{m 1}=G_{m 2} \frac{C_{2}}{C_{L}}
\end{gathered}
$$

7. $C_{L}$ must be $\gg C_{T}$; otherwise it can be optimized for power.

$$
P=\frac{C_{L}}{V_{L}} V_{d d} Q_{\text {max }} f_{\text {center }}
$$

\subsection{Ratio of Transconductances}

The $0.18 \mu m \mathrm{C}^{4}$ was designed for unity gain with $Q_{\max }=2.9$. Figure 2.5 shows the effect the transconductance ratio has on the $\mathrm{AC}$ characteristics of the $\mathrm{C}^{4}$. The gain and $\mathrm{Q}$ are transconductance dependent and given by

$$
\begin{gathered}
A_{v}=-\frac{C_{1}}{C_{2}} \frac{1}{1+\frac{C_{L}}{C_{2}} \frac{G_{m 2}}{G_{m 1}}} \\
Q=\frac{\sqrt{C_{O} C_{T}-C_{2}{ }^{2}}}{C_{L} \sqrt{\frac{G_{m 2}}{G_{m 1}}}+C_{2} \sqrt{\frac{G_{m 1}}{G_{m 2}}}}
\end{gathered}
$$




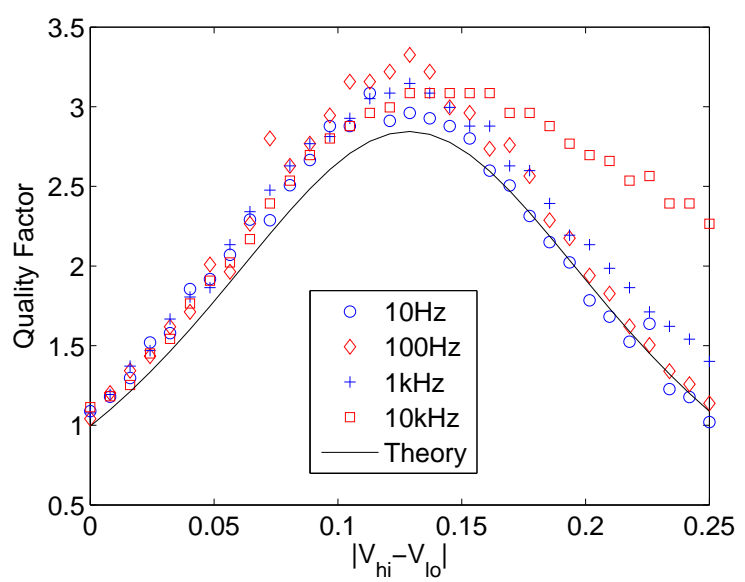

(a)

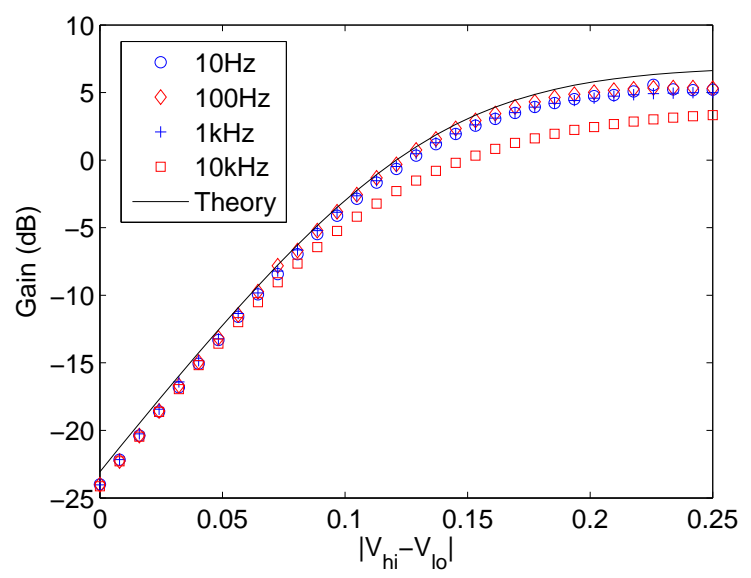

(b)

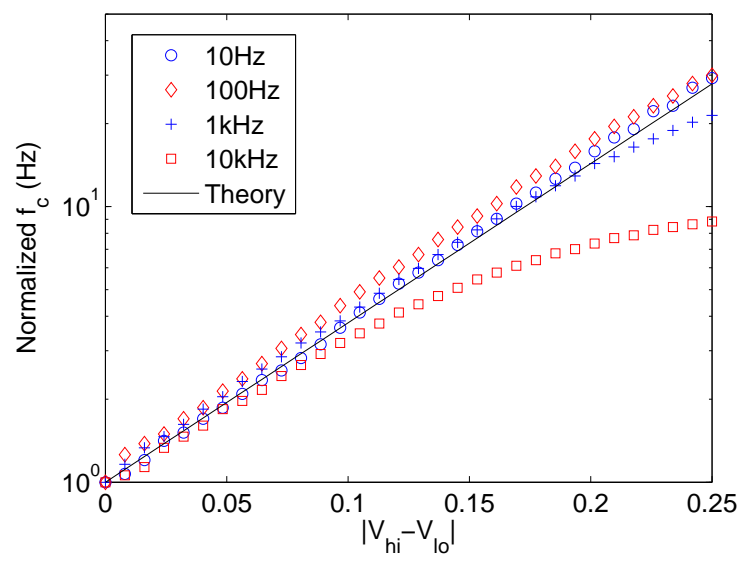

(c)

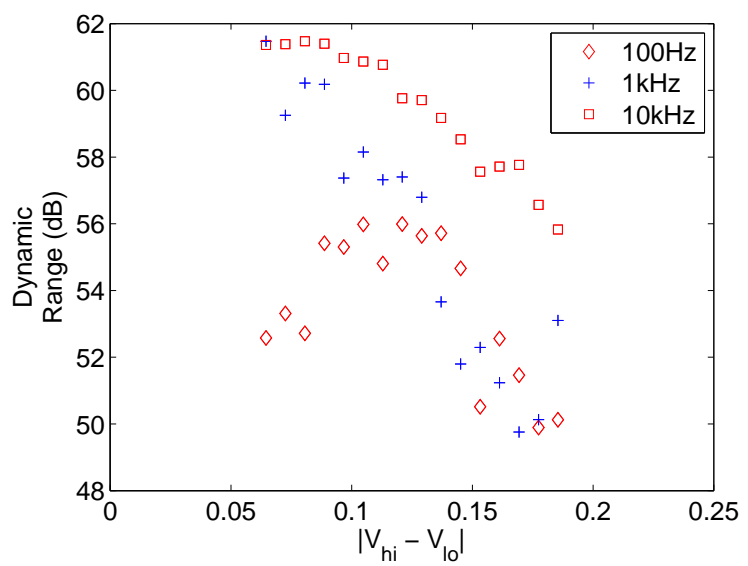

(d)

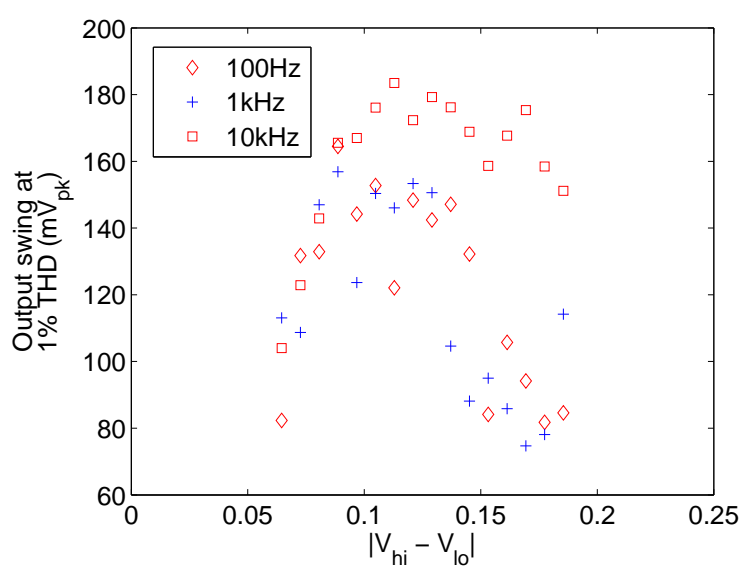

(e)

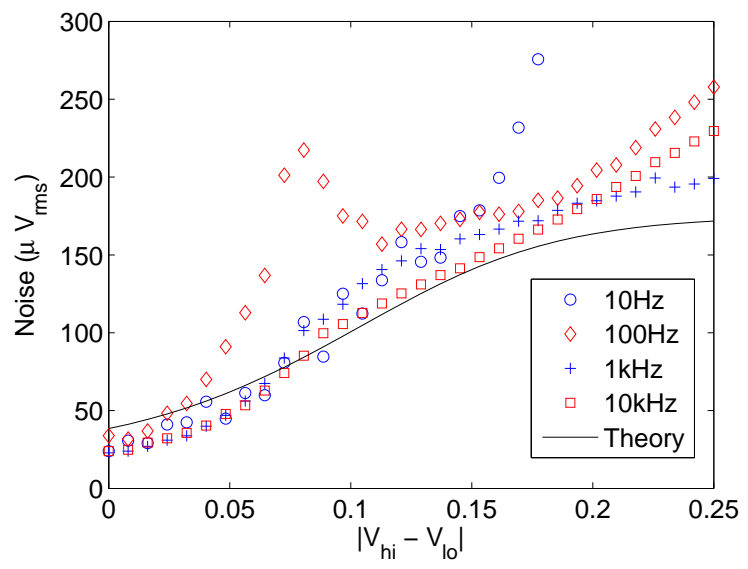

(f)

Figure 2.5: Effect of $G_{m 1} / G_{m 2}$ on (a) Q, (b) Gain, (c) Center frequency, (d) Dynamic range, (e) Output swing, and (f) Noise 
The maximum gain is $A_{v, \max }=-C_{1} / C_{2}$ and occurs for $G_{m 1} \gg G_{m 2}$. $Q_{\max }$ occurs when $C_{2} / C_{L}=G_{m 2} / G_{m 1}$ and is given by

$$
Q_{\max }=\frac{1}{2} \sqrt{\frac{C_{T} C_{O}-C_{2}{ }^{2}}{C_{L} C_{2}}}
$$

When biased for $Q_{\max }$, the gain is $-C_{1} / 2 C_{2}$, which is half of the maximum gain. In fig. 2.5 the maximum $\mathrm{Q}$ is approximately 3 and occurs with unity gain. The gain increases with the ratio of bias currents until reaching $6 d B$ (twice $0 d B$ ). Note that for the $10 \mathrm{kHz}$ case, $G_{m 1}$ has gone out of sub-threshold and its transconductance is no longer linearly proportional to its bias current. This is why the $10 \mathrm{kHz}$ curves have different characteristics.

Figure 2.5(d) shows how the dynamic range is dependent on the ratio of transconductances. Here the dynamic range is defined as

$$
\text { Dynamic Range }=\frac{\text { Output swing with } 1 \% \text { THD }}{\text { Output referred noise }}
$$

The noise increases with $G_{m 1} / G_{m 2}$ due to the increasing gain and bandwidth. For the $100 \mathrm{~Hz}$ case the noise is higher for $\left|V_{h i}-V_{l o}\right| \approx 80 \mathrm{mV}$ because $60 \mathrm{~Hz}$ line noise falls in the passband. The output swing is largely independent of $G_{m 1} / G_{m 2}$, which is illustrated in fig. 2.6.

\subsection{Tuning Range}

For audio processing, the filters must be tunable from $20 \mathrm{~Hz}$ to $20 \mathrm{kHz}$. Figure 2.8 demonstrates the ability to bias the $\mathrm{C}^{4}$ from $5 \mathrm{~Hz}$ to $50 \mathrm{kHz}$ with a gain of $0 \mathrm{~dB}$ or $4 \mathrm{~dB}$.

When biased in sub-threshold, the transconductance of an OTA is exponentially dependent on its bias voltage. This allows the filters to have logarithmic frequency spacing simply by varying the bias voltages linearly as seen in fig. 2.7. Here the biases were stepped linearly to emulate a 32 channel filter bank. The gain begins to drop at the higher frequency biases because the OTA's are dropping out of sub-threshold.

\subsection{Specifications}

The objective was to design a bandpass filter with a $60 d B$ dynamic range which is tunable across the auditory range and has a $\mathrm{Q}$ of 2.9 at unity gain. This section summarizes the 

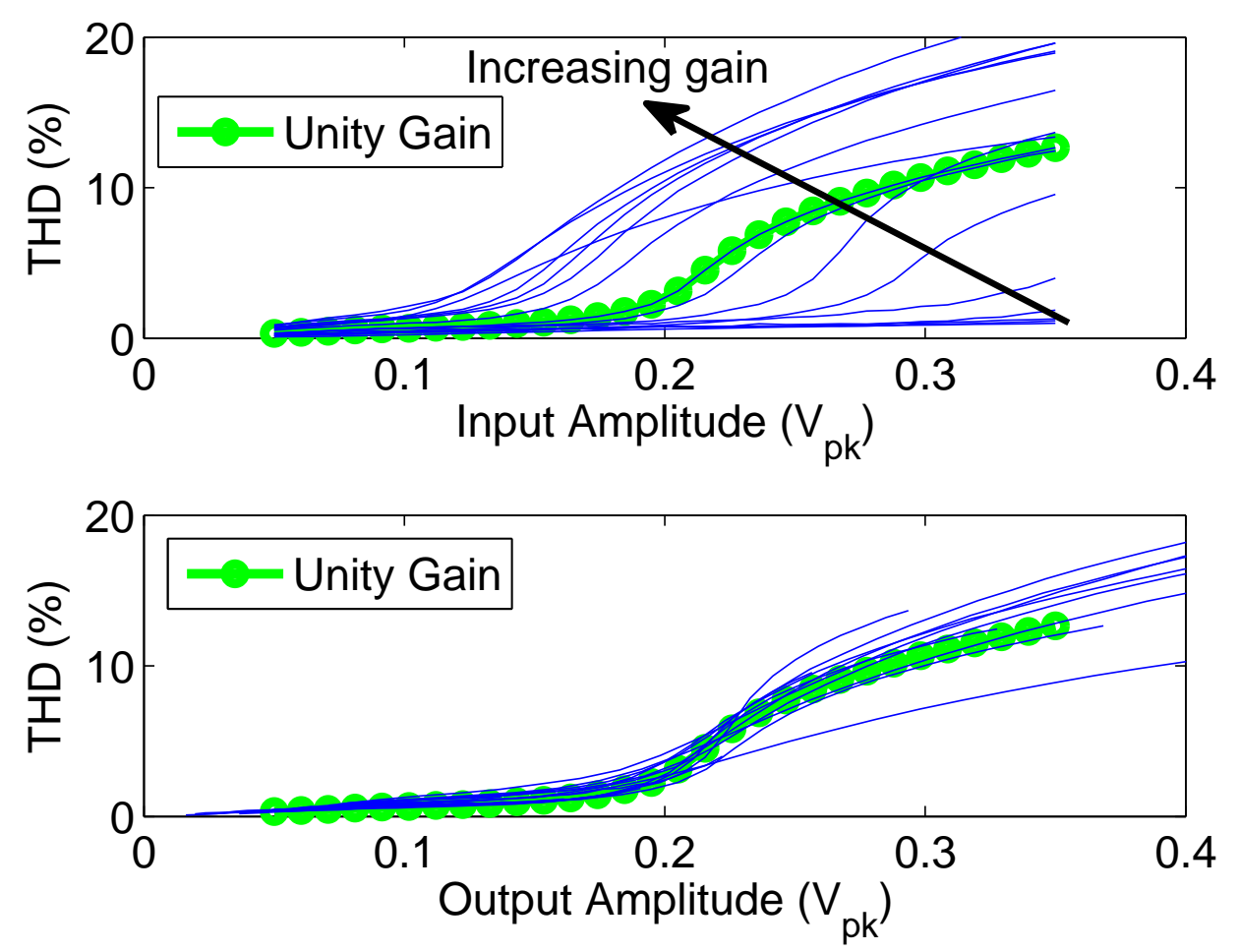

Figure 2.6: THD for different transconductance ratios. (a) Versus input swing (b) versus output swing. Note that the output swing is independent of passband gain.

measured performance of the filter at four representative frequencies: $20 \mathrm{~Hz}, 200 \mathrm{~Hz}, 2 \mathrm{kHz}$, and $20 \mathrm{kHz}$. Figure 2.9 shows the frequency response and noise for these biasing conditions. The filters are biased at unity gain with a $\mathrm{Q}$ of 3 . The THD as a function of input amplitude for each tap is shown in fig. 2.10(a). The THD exceeds $1 \%$ at around $120 m V_{p k}$. The output spectrum for a $100 m V_{p k}$ sine wave for each biasing condition is shown in fig. 2.10(b). The $\mathrm{C}^{4}$ performance is summarized in table 2.2 . 

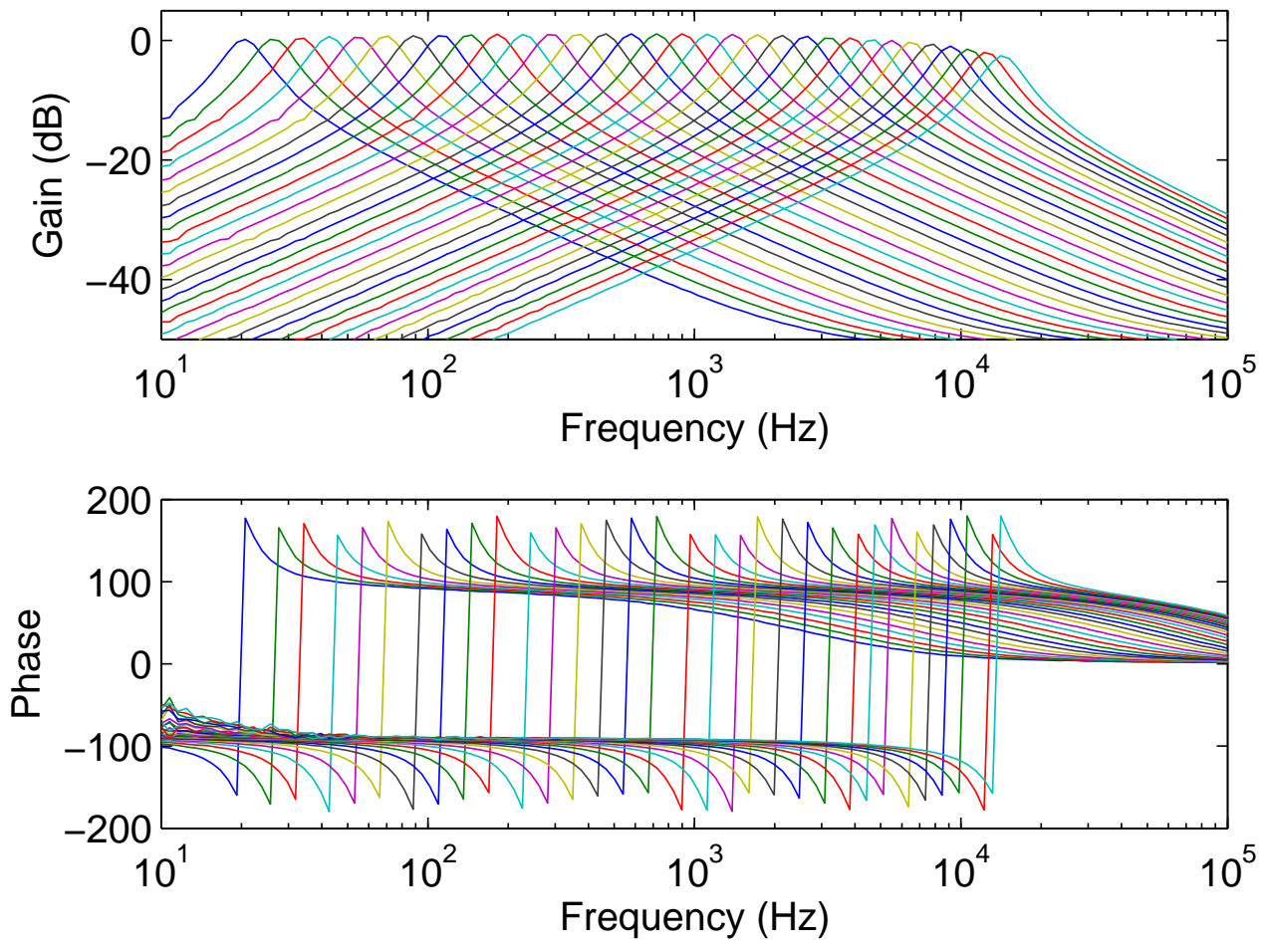

Figure 2.7: Emulating a 32 channel filter bank by a linear stepping of bias voltages.

Table 2.2: $0.18 \mu m \mathrm{C}^{4}$ characteristics

\begin{tabular}{c|cccc} 
& $\mathbf{2 0} \mathbf{H z}$ & $\mathbf{2 0 0} \mathbf{H z}$ & $\mathbf{2} \mathbf{~ k H z}$ & $\mathbf{2 0} \mathbf{~ k H z}$ \\
\hline Gain $(\mathrm{dB})$ & 0.13458 & 0.032579 & -0.24219 & 0.021603 \\
Q & 3.0465 & 2.993 & 3.0667 & 2.9688 \\
Center Frequency (Hz) & 21.614 & 206.96 & 2080.3 & 20420 \\
THD (\%) - 100mV $V_{p k}$ & 0.53655 & 0.84547 & 0.73581 & 0.65976 \\
Amplitude for 1\% THD & 0.12347 & 0.11122 & 0.12959 & 0.17857 \\
Integrated Noise $(\mu V)$ & 173.95 & 152.74 & 148.49 & 195.16 \\
Dynamic Range (dB) & 57.023 & 57.245 & 58.817 & 59.228
\end{tabular}




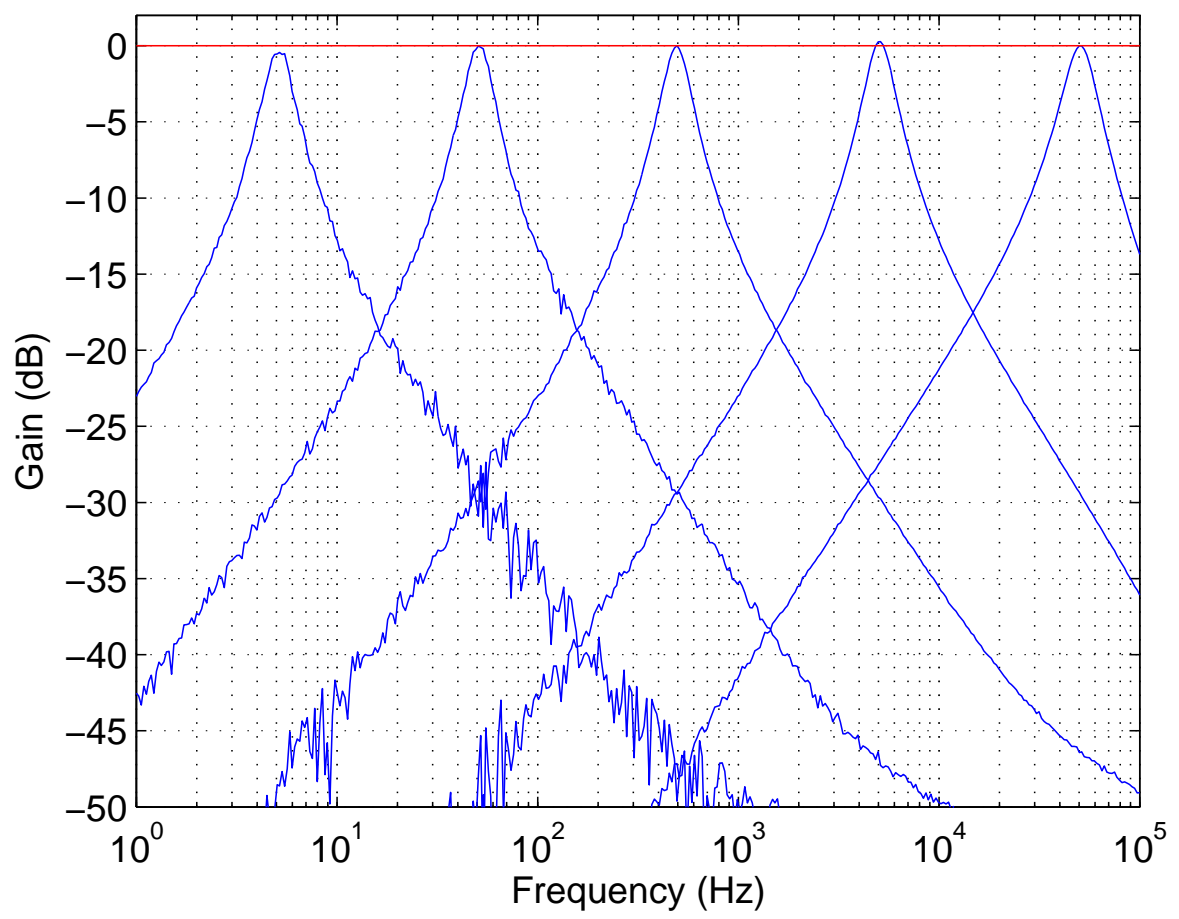

(a)

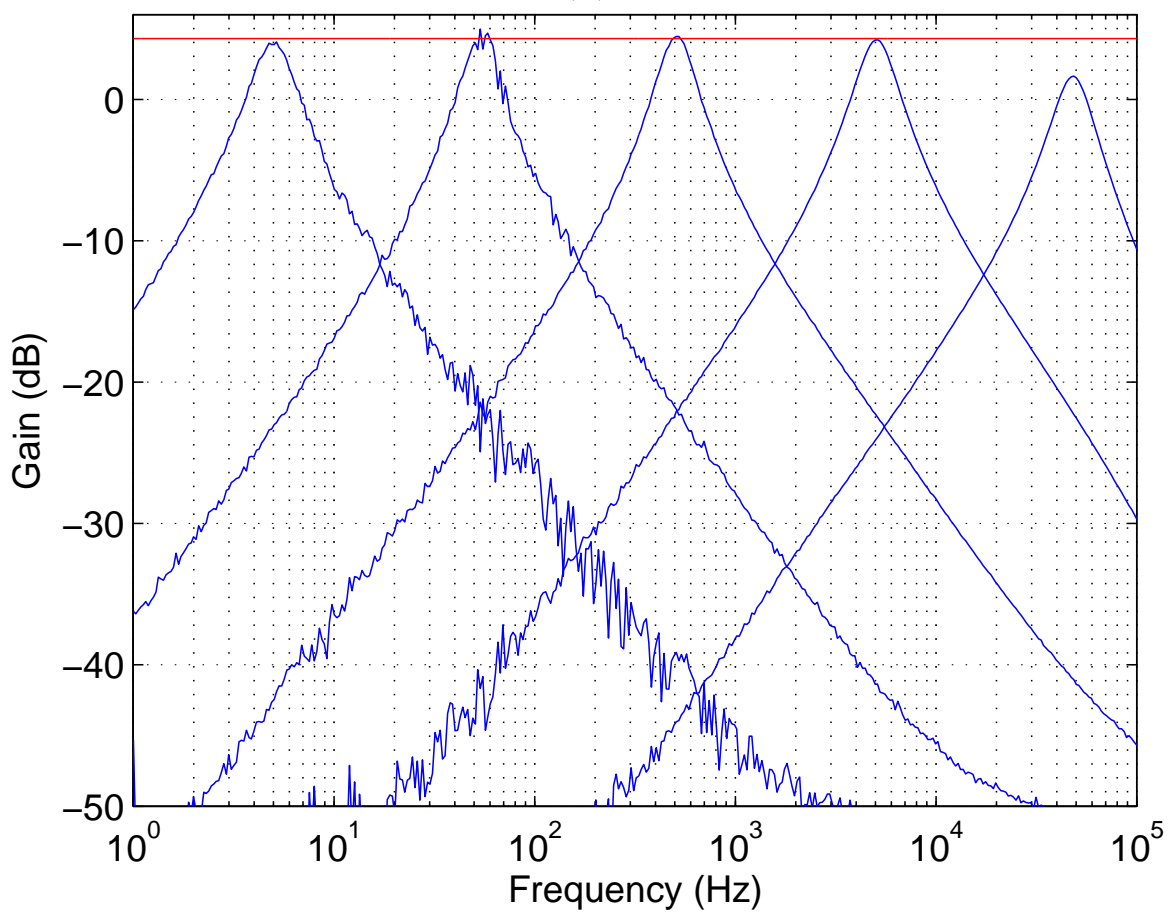

(b)

Figure 2.8: $\mathrm{C}^{4}$ biased from $5 \mathrm{~Hz}$ to $50 \mathrm{kHz}$ with a gain of (a) $0 \mathrm{~dB}$ (b) $4 d B$ 

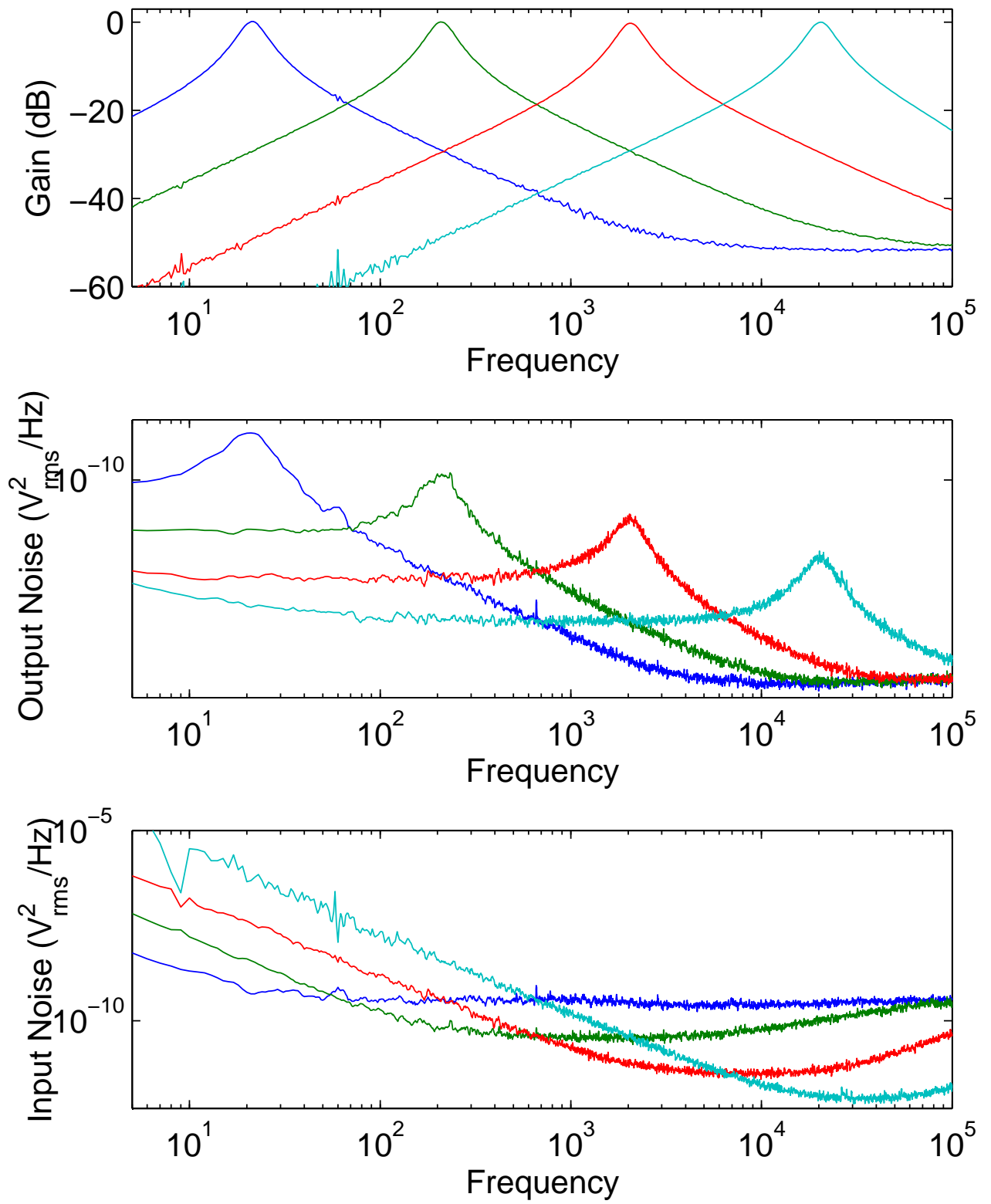

Figure 2.9: (a) Frequency response (b) Output referred noise (c) Input referred noise 


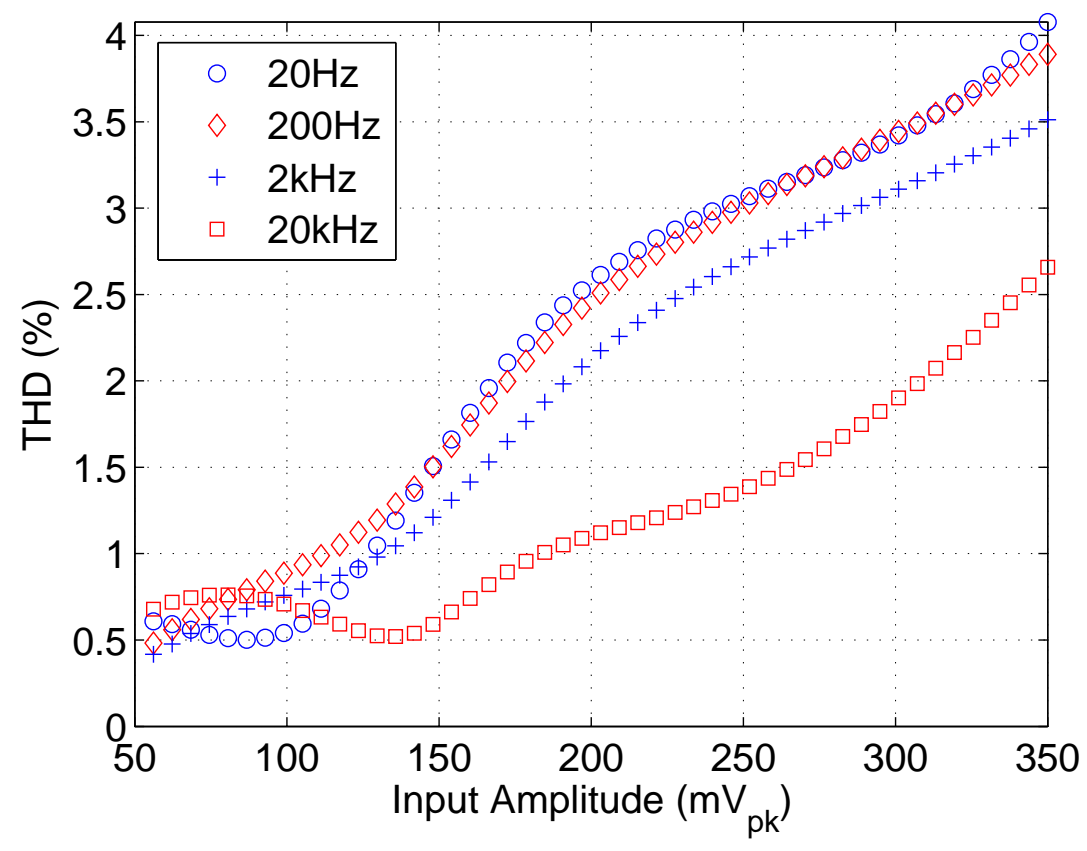

(a)
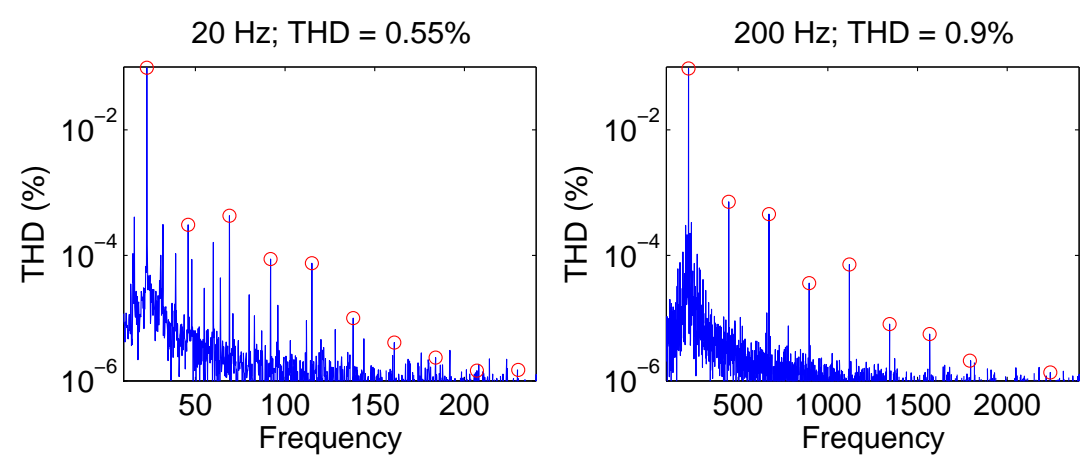

$2 \mathrm{kHz} ; \mathrm{THD}=0.76 \%$
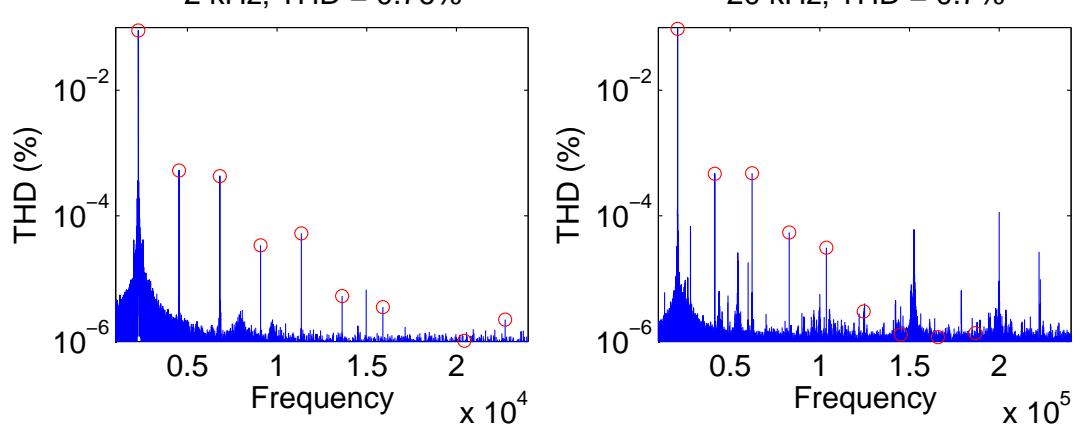

(b)

Figure 2.10: (a) THD vs. amplitude for four center frequencies. (b) Output spectrum in response to a $100 m V_{p k}$ sine wave at the center frequencies for filters biased at: $20 \mathrm{~Hz}, 200$ $\mathrm{Hz}, 2 \mathrm{khz}$, and $20 \mathrm{kHz}$. 


\section{Chapter 3}

\section{Magnitude Detector}

Magnitude detectors track the power of a signal and are important for spectral decomposition, automatic gain control, demodulation, and RMS-to-DC conversion. This chapter details the development of a novel magnitude detector circuit for sub-band power detection in an analog audio processing front-end. This circuit has been fabricated (and tested) in $0.18 \mu \mathrm{m}$ and $0.5 \mu \mathrm{m}$ processes. Figure 3.1 illustrates the structure of this magnitude circuit. The first stage performs a piecewise nonlinear voltage-to-current conversion and integrates the current on a capacitor. The output of the first stage tracks the RMS of the input with significant ripple. This ripple is removed in the second stage with a nonlinear low-pass filter. The filter's time constant increases for smaller amplitudes to achieve a trade-off between ripple and tracking ability. The magnitude detector has a $68 d B$ dynamic range and consumes less than a $\mu W$.

Section 3.1 details the important characteristics of magnitude detectors. In section 3.2 some common peak detector architectures are discussed. Section 3.3 presents a peak detector with amplitude dependent decay rates, this detector forms the first stage of our magnitude detector. The nonlinear filter used in the second stage of the magnitude detector is presented in section 3.4. In section 3.5 the complete magnitude circuit is discussed. The chapter ends with a tuning algorithm for the circuit. 


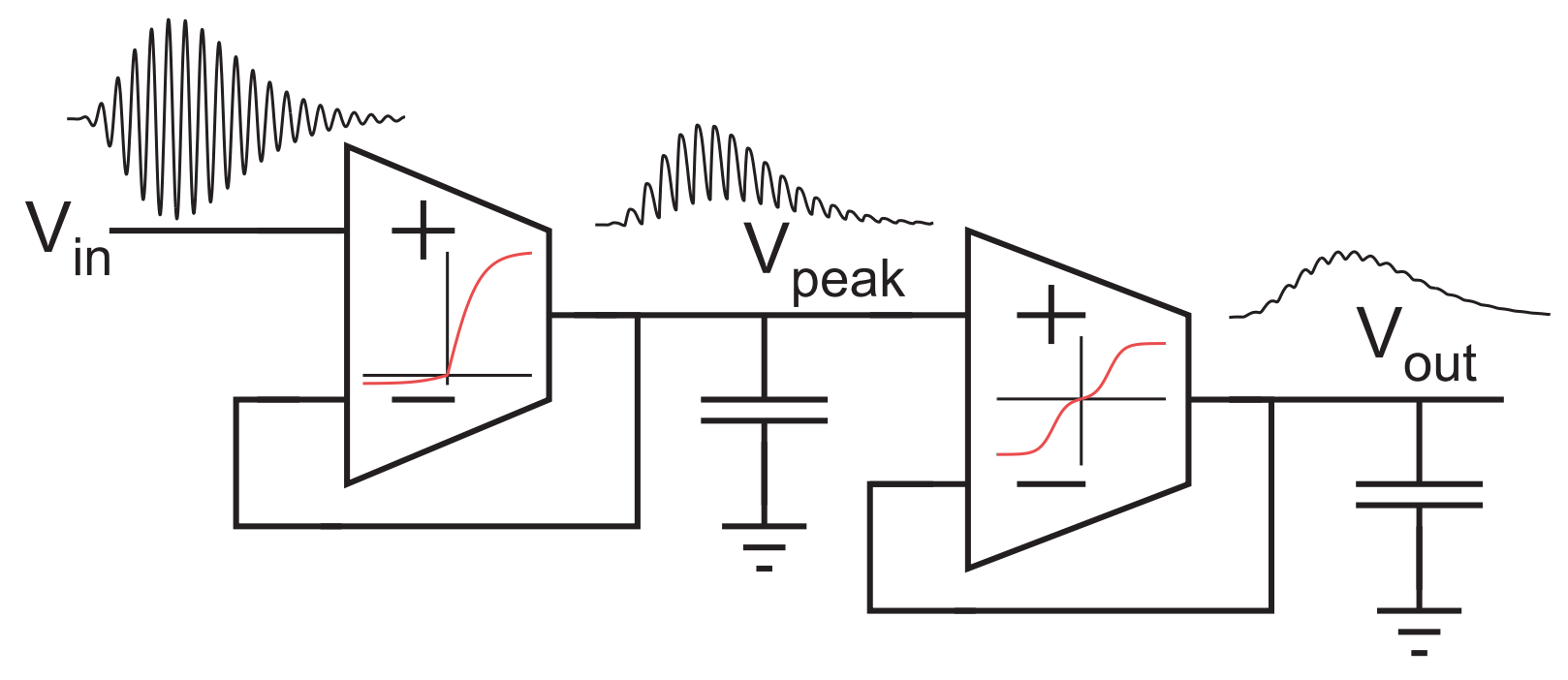

Figure 3.1: Block diagram of proposed magnitude circuit. The first stage performs peak detection with adjustable attack and decay rates and the second stage averages the output.

\subsection{Magnitude Detector Characteristics}

A magnitude detector's desirability depends on its dynamic range, accuracy, tracking capability, size and power consumption. Accuracy is how close the output is to the true magnitude and is affected by the amplitude of the input signal (higher amplitude causes more ripple), in addition to noise and linearity [15]. Tracking is the ability to follow timevarying changes in the magnitude of the signal. There is a trade-off between tracking and ripple (accuracy).

A magnitude detector may track either the amplitude or RMS of a signal. Our specification for the circuit was to follow the RMS.

\subsubsection{RMS}

A common metric for measuring the power of a signal is root mean square (RMS) which is defined as

$$
R M S=\sqrt{\int x(t)^{2} d t}
$$

There are three steps in equation 3.1, square (rectification), mean (time average), and root (normalization). A signal's RMS is the DC voltage that will produce the same quantity of 
heat when applied to an equal load.

\subsubsection{Tracking}

When dealing with time-varying signals there is a trade-off in the integration portion of the RMS computation. A large time constant is not able to track large amplitude changes while a small time constant will have ripple from the carrier wave, this trade-off is illustrated in fig. 3.2. In order to accurately track the RMS with minimal ripple the integration window should fulfill the relationship

$$
\frac{1}{2 \pi f_{\text {mod,max }}} \gg \tau \gg \frac{1}{2 \pi f_{\text {car,min }}}
$$

where $f_{\text {mod,max }}$ is the highest frequency of interest in the modulating signal and $f_{c a r, m i n}$ is the lowest frequency in the carrier signal. This is particularly difficult for speech signals which have less than an order of magnitude difference between $f_{\text {mod,max }}$ and $f_{\text {car,min }}$.

When performing an RMS on sub-bands in a constant-Q filter bank the integration window for each sub-band is related to the center frequency and bandwidth. Rewriting the above equation for a filter bank yields

$$
\frac{Q}{2 \pi f_{c}} \gg \tau \gg \frac{1}{2 \pi f_{c}}
$$

where $f_{c}$ is the center frequency of the band and $Q$ is the quality factor given by $f_{c} /$ bandwidth .

\subsection{Magnitude Detector Structure}

Most magnitude detectors follow the structure of fig. 3.3(a). Rectification may come in the form of full-wave [16], half-wave, and squaring [17]. The problem with squaring is that it increases the dynamic range of the signal, putting stricter constraints on the circuits which process the squared signal [18].

\subsubsection{Attack and Decay}

Peak detectors work by following the signal up to the peak (attack) then falling slower than the signal (decay), this is illustrated in fig. 3.3(b). The attack should be fast enough 


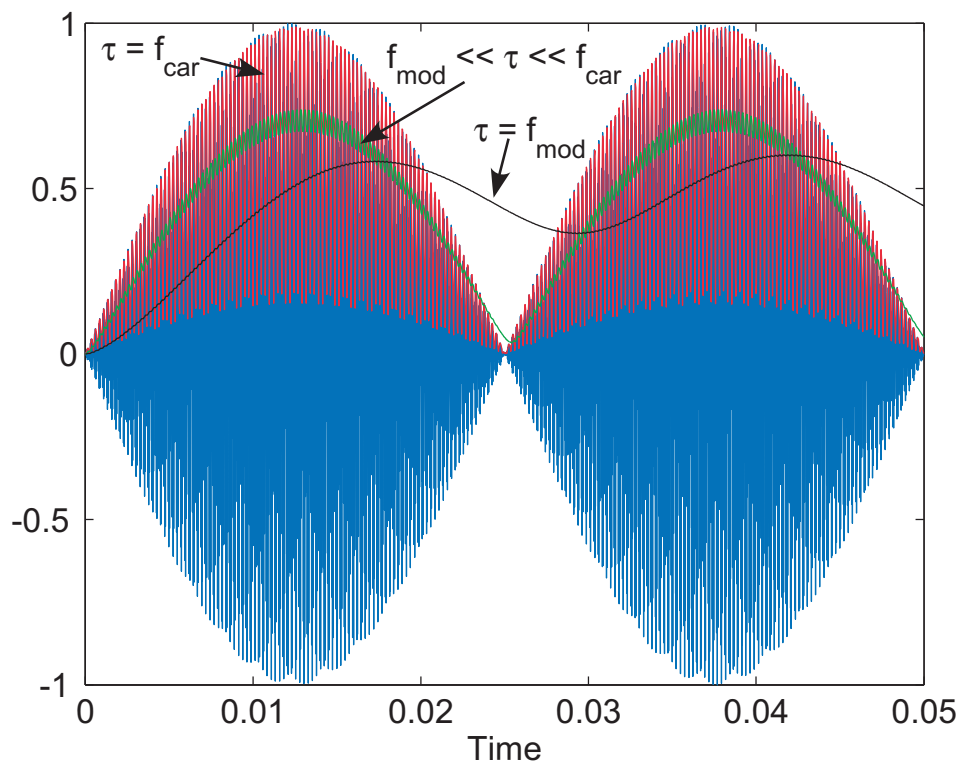

(a)

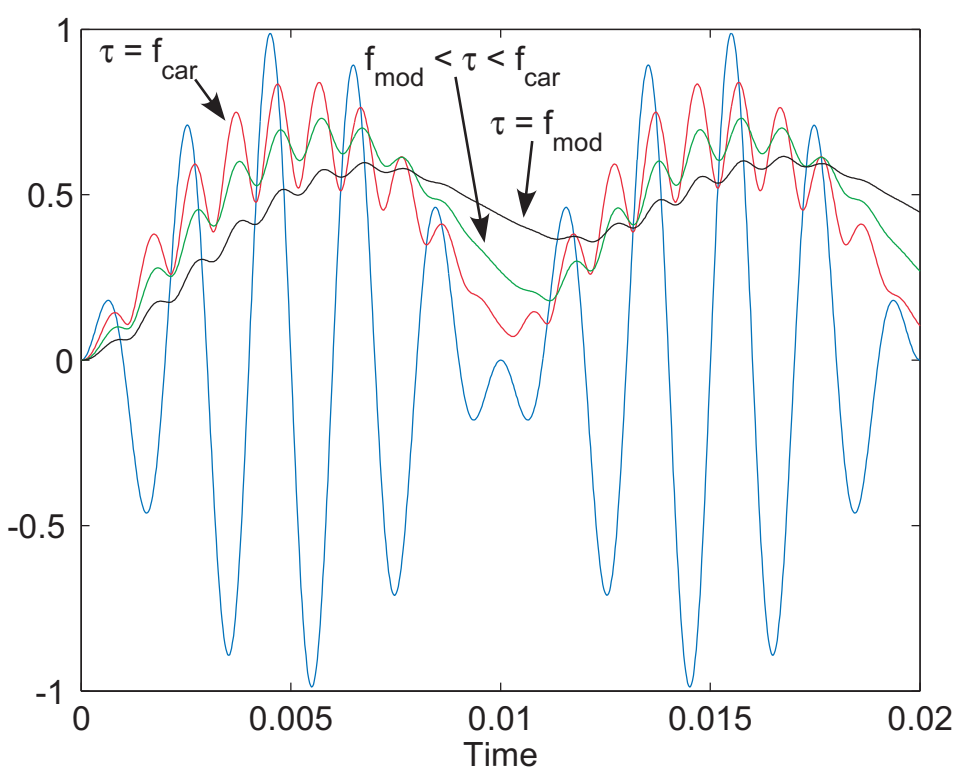

(b)

Figure 3.2: RMS computed with different time constants. (a) $f_{\text {car }} / f_{\text {mod }}=1000$ (b) $f_{\text {car }} / f_{\text {mod }}=10$ 


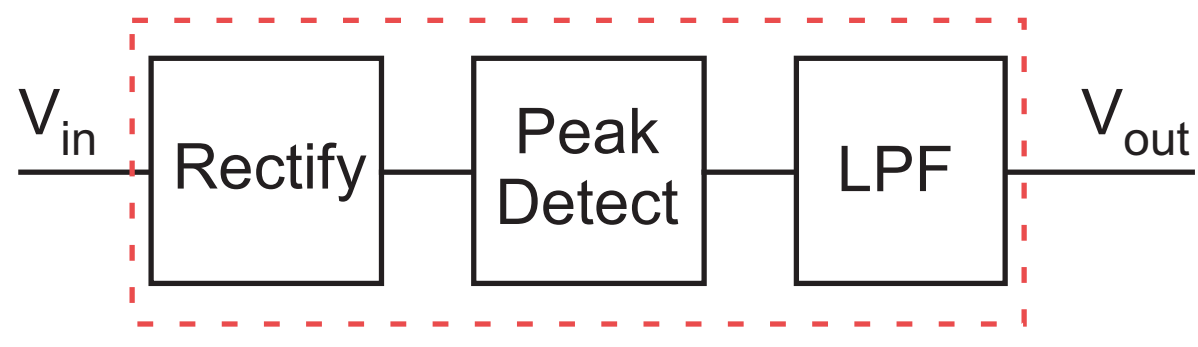

(a)

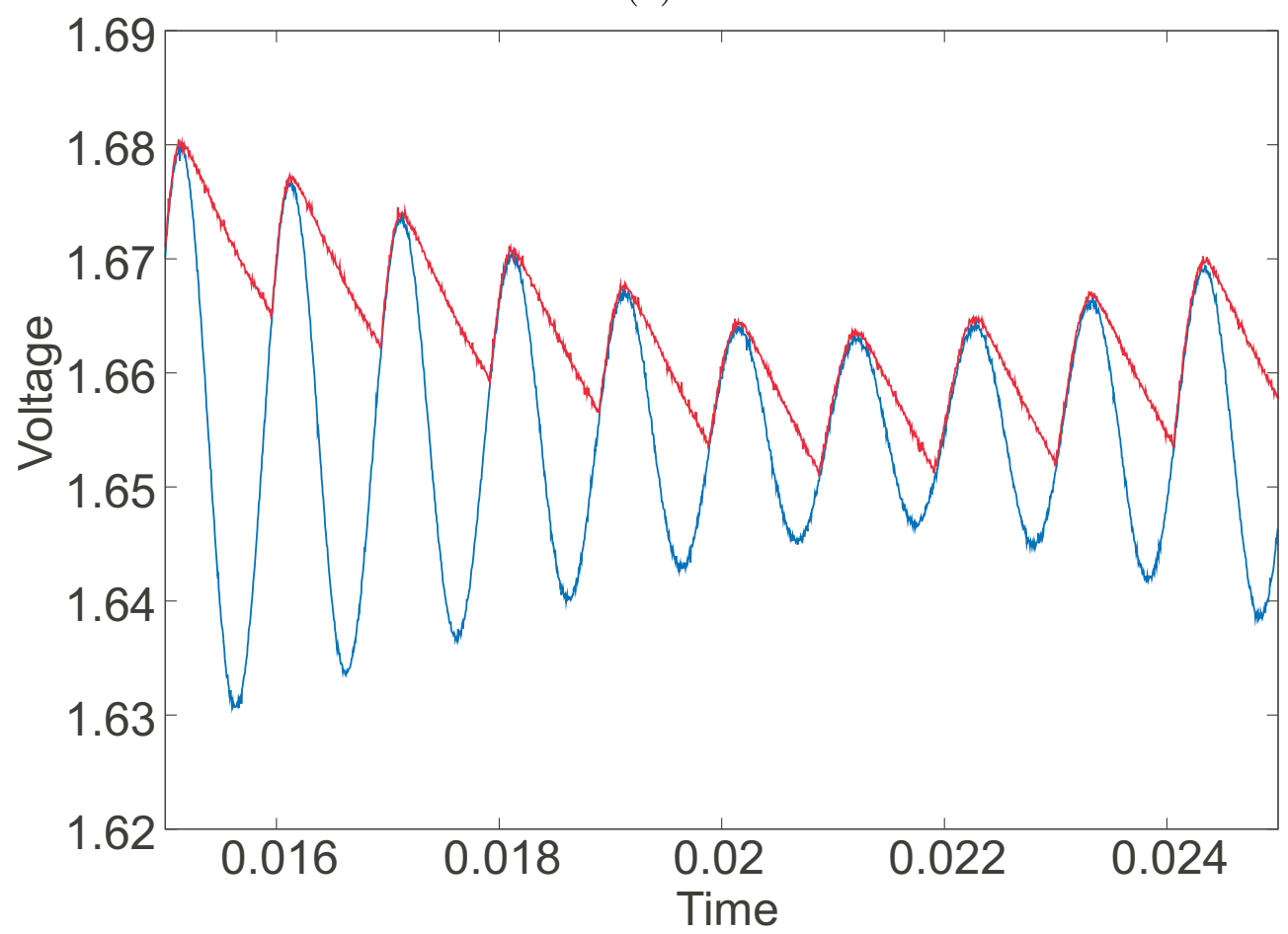

(b)

Figure 3.3: (a) A typical magnitude detector consists of rectification, peak detection, and averaging. (b) Measured response of a typical peak detector to an amplitude modulated sine wave. The peak detector follows the wave up to the peak, then decays until the next upward going portion. 


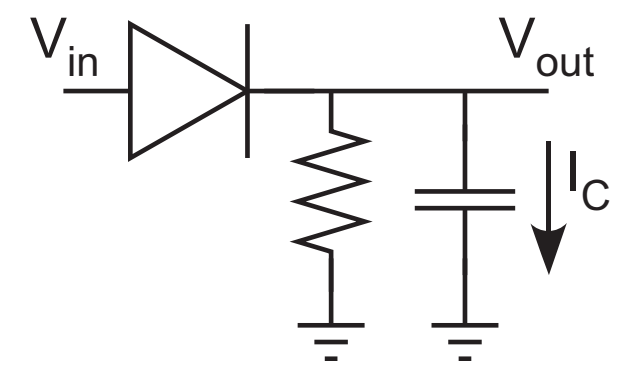

(a)

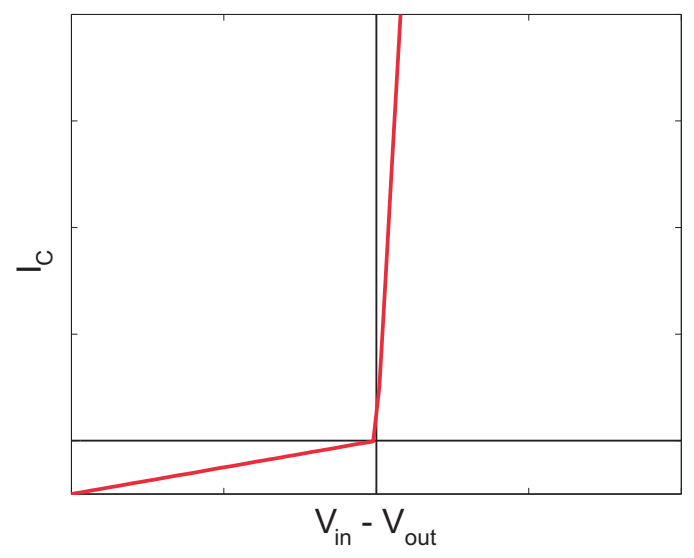

(b)

Figure 3.4: (a) Classic diode detector uses a diode for voltage-to-current conversion and rectification, while a resistor provides decay. (b) Current onto the capacitor as a function of $V_{\text {in }}-V_{\text {out }}$.

to slew up to the peak. The decay should be fast enough to not miss peaks but slow enough that it does not bottom out at small amplitudes. In section 3.3, it is shown that an amplitude dependent decay rate allows the detector to operate over a large dynamic range with fast amplitude variations.

\subsubsection{Classic Diode Detector}

To illustrate the concept of a peak detector, let's examine the earliest and most recognizable envelope detector, the diode detector seen in fig. 3.4(a). We will treat the diode as a switch with some on resistance $R_{a t k}$ and a zero-volt turn-on voltage. The circuit has two regions of operation, when the diode is on it occupies the attack region, when the diode is off it is in the decay region. The attack region occurs when the input exceeds the output, and the capacitor charges to follow the input. While the input is less than the output it operates in the decay region, and the output falls while charge bleeds off through the resistor. The current onto the capacitor $\left(I_{C}\right)$ is a piecewise linear function of $V_{\text {in }}$ and $V_{\text {out }}$ given by

$$
I_{C}= \begin{cases}\frac{V_{\text {in }}-V_{\text {out }}}{R_{\text {otk }}}-\frac{V_{\text {out }}}{R_{\text {dec }}} & : V_{\text {in }}-V_{\text {out }}>0 \\ -\frac{V_{\text {out }}}{R_{\text {dec }}} & : V_{\text {in }}-V_{\text {out }}<0\end{cases}
$$

$I_{C}$ is plotted in fig. $3.4(\mathrm{~b})$. 


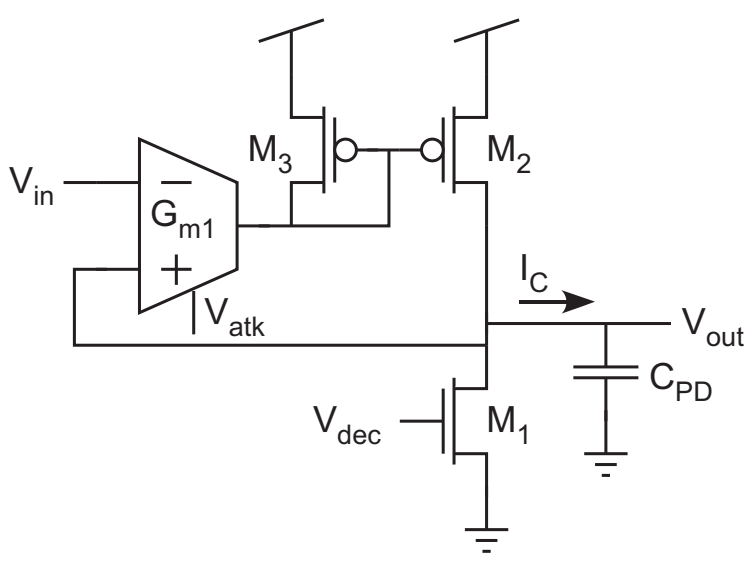

(a)

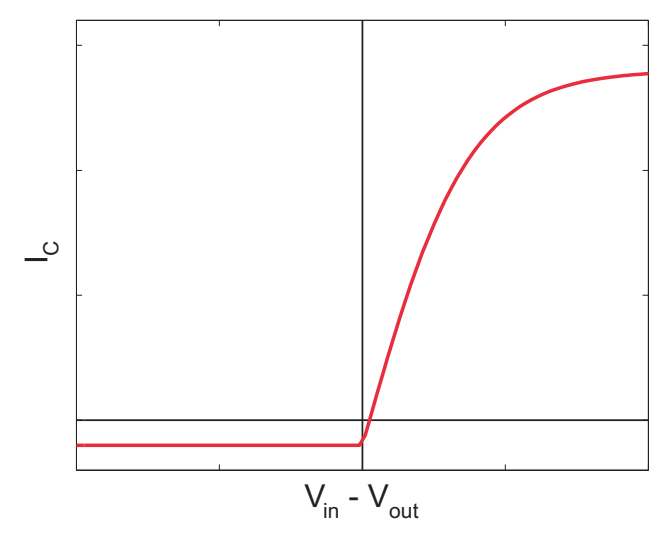

(b)

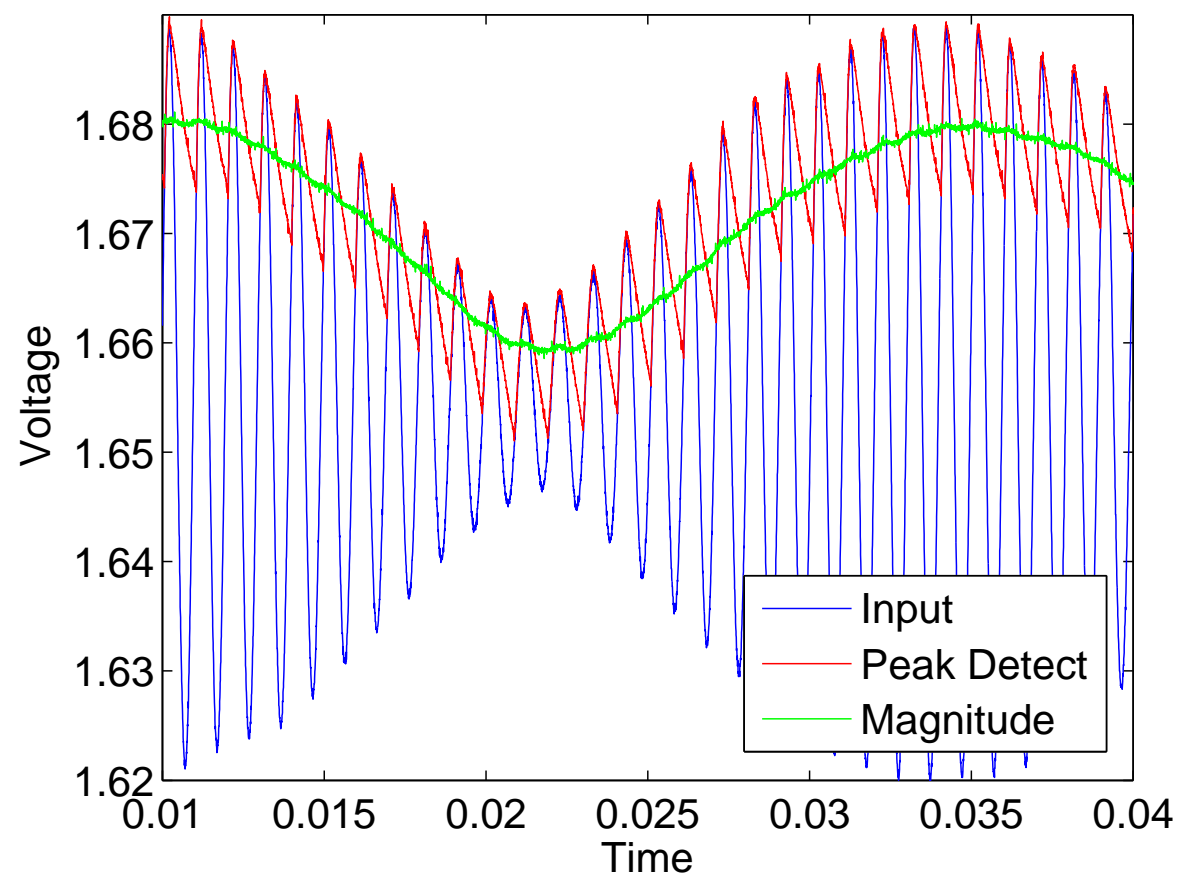

(c)

Figure 3.5: (a) In the constant decay peak detector, the attack current is the half-wave rectified current from the OTA. Transistor $M_{1}$ is a current sink for $I_{d e c}$. (b) Current onto capacitor as a function of $V_{\text {in }}-V_{\text {out }}$. (c) Demonstration of the constant decay peak detector. The input is an amplitude modulated sine wave. The magnitude trace is the response of the second stage (section 3.4) of our magnitude detector. 


\subsection{3 $G_{m}-C$ Magnitude Detectors}

The diode detector circuit is inappropriate for CMOS integrated circuits due to it's use of resistors and diodes. Most CMOS implementations have used OTA's for voltage-to-current conversion. Figure 3.5(a) shows a common CMOS peak detector which we will call the constant decay peak detector. The current mirror formed by $M_{2}$ and $M_{3}$ half-wave rectifies $G_{m}\left(V_{\text {in }}-V_{\text {out }}\right)$ onto the capacitor while transistor $M_{1}$ acts as a current source and sets the decay. The current onto the capacitor is described by

$$
I_{C}= \begin{cases}I_{\text {atk }} \tanh \left(\frac{\kappa}{2 U_{T}}\left(V_{\text {in }}-V_{\text {out }}\right)\right)-I_{\text {dec }} & : V_{\text {in }}-V_{\text {out }}>0 \\ -I_{\text {dec }} & : V_{\text {in }}-V_{\text {out }}<0\end{cases}
$$

where $I_{a t k}$ is the bias current of the OTA and $I_{d e c}$ is the bias current of $M_{1}$. $I_{C}$ is plotted in fig. 3.5(b). Figure 3.5(c) demonstrates the operation of the circuit in response to an amplitude modulated sine wave. Note how the peak detector output decays linearly, this is because $I_{C}$ is constant for $V_{i n}-V_{\text {out }}<0$.

\subsection{Adaptive Decay Peak Detector}

The problem with the constant decay peak detector is that the decay rate is only appropriate for a small range of amplitudes. If the peak detector is to work for a large dynamic range then the decay rate must adapt to the amplitude. This can be accomplished by mirroring the top half of the circuit so the decay is a function of $V_{\text {in }}-V_{\text {out }}$. Figure 3.6(a) shows the resulting circuit for which the current onto the capacitor (fig. 3.6(b)) can be written as

$$
I_{C}= \begin{cases}I_{\text {atk }} \tanh \left(\frac{\kappa}{2 U_{T}}\left(V_{1}-V_{2}\right)\right) & : V_{\text {in }}-V_{\text {out }}>0 \\ I_{\text {dec }} \tanh \left(\frac{\kappa}{2 U_{T}}\left(V_{1}-V_{2}\right)\right) & : V_{\text {in }}-V_{\text {out }}<0\end{cases}
$$

where $I_{a t k}$ is the bias current of $G_{m 1}$ and $I_{d e c}$ is the bias current of $G_{m 2}$. The circuit's response to an amplitude modulated sine wave is shown in fig. 3.6(c). Note how the peak detect output decays more for larger amplitudes. Figure 3.7 shows a comparison of the constant and adaptive decay circuits. The input is downward steps of varying sizes. For the smallest step the constant decay immediately bottoms out while the adaptive decay has 


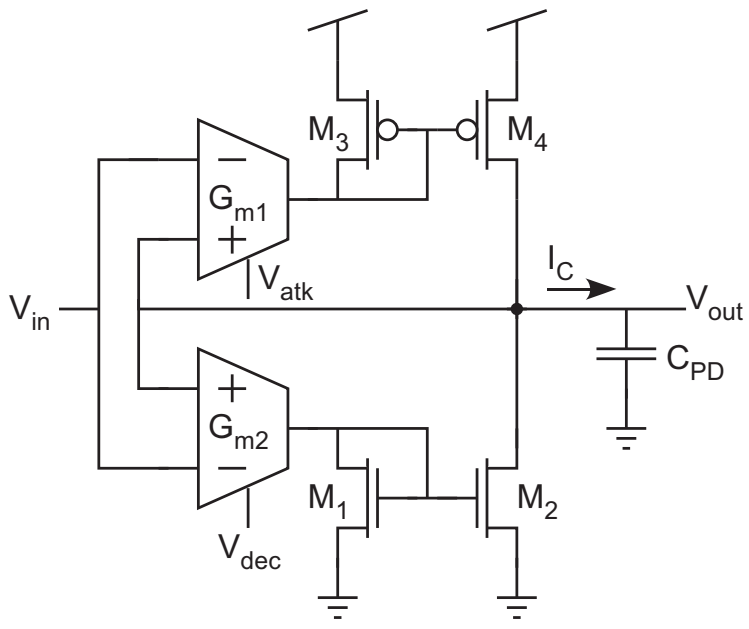

(a)

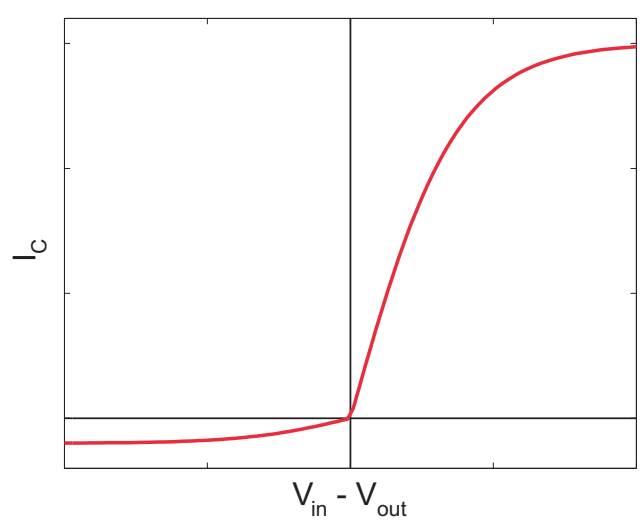

(b)

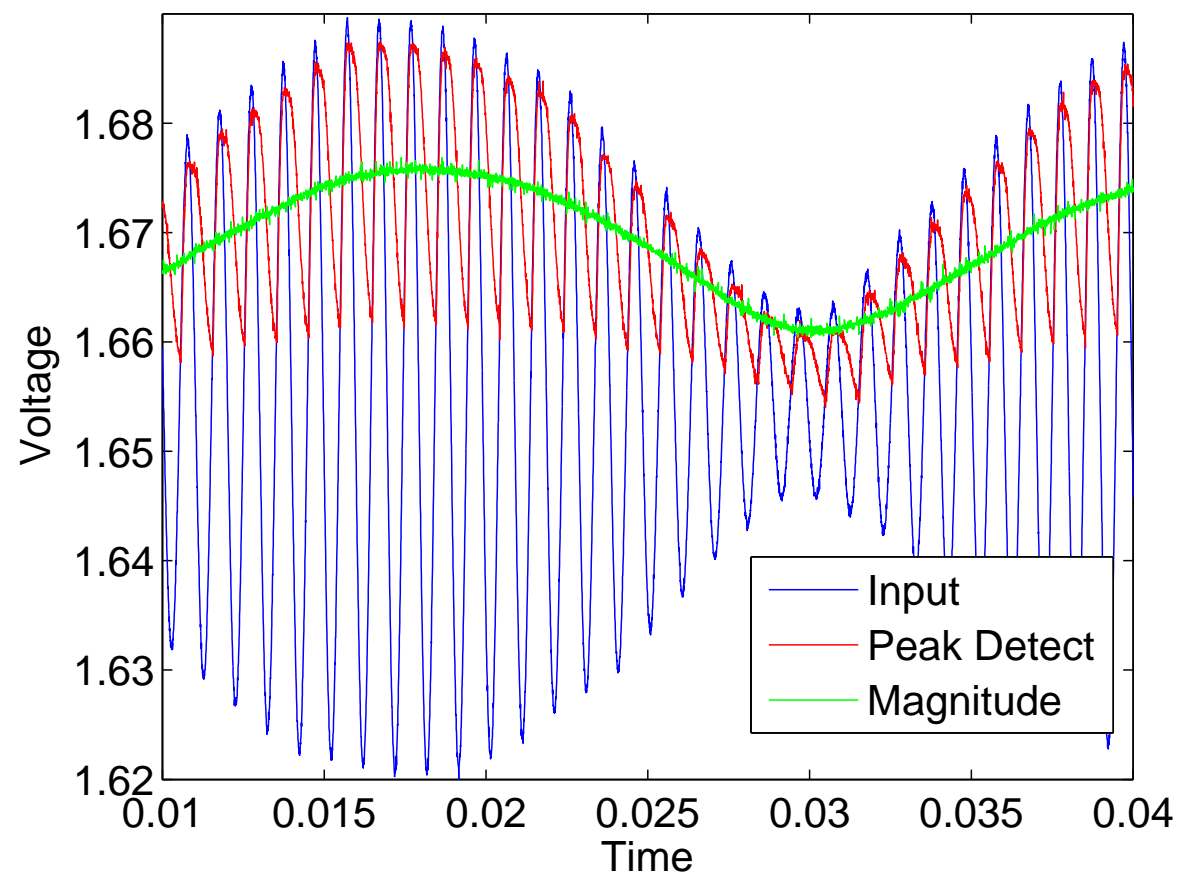

(c)

Figure 3.6: (a) The adaptive decay peak detector. $G_{m 1}, M_{3}$, and $M_{4}$ generate the attack current while $G_{m 2}, M_{1}, M_{2}$ generate the decay current. For this circuit the attack and decay are both a function of $V_{\text {in }}-V_{\text {out }}$. The OTA bias currents provide direct control over the attack and decay rates. (b) Current onto capacitor as a function of $V_{\text {in }}-V_{\text {out }}$. (c) Adaptive decay peak detector response to an amplitude modulated sine wave. The magnitude trace is the response of the second stage (section 3.4) of our magnitude detector. 


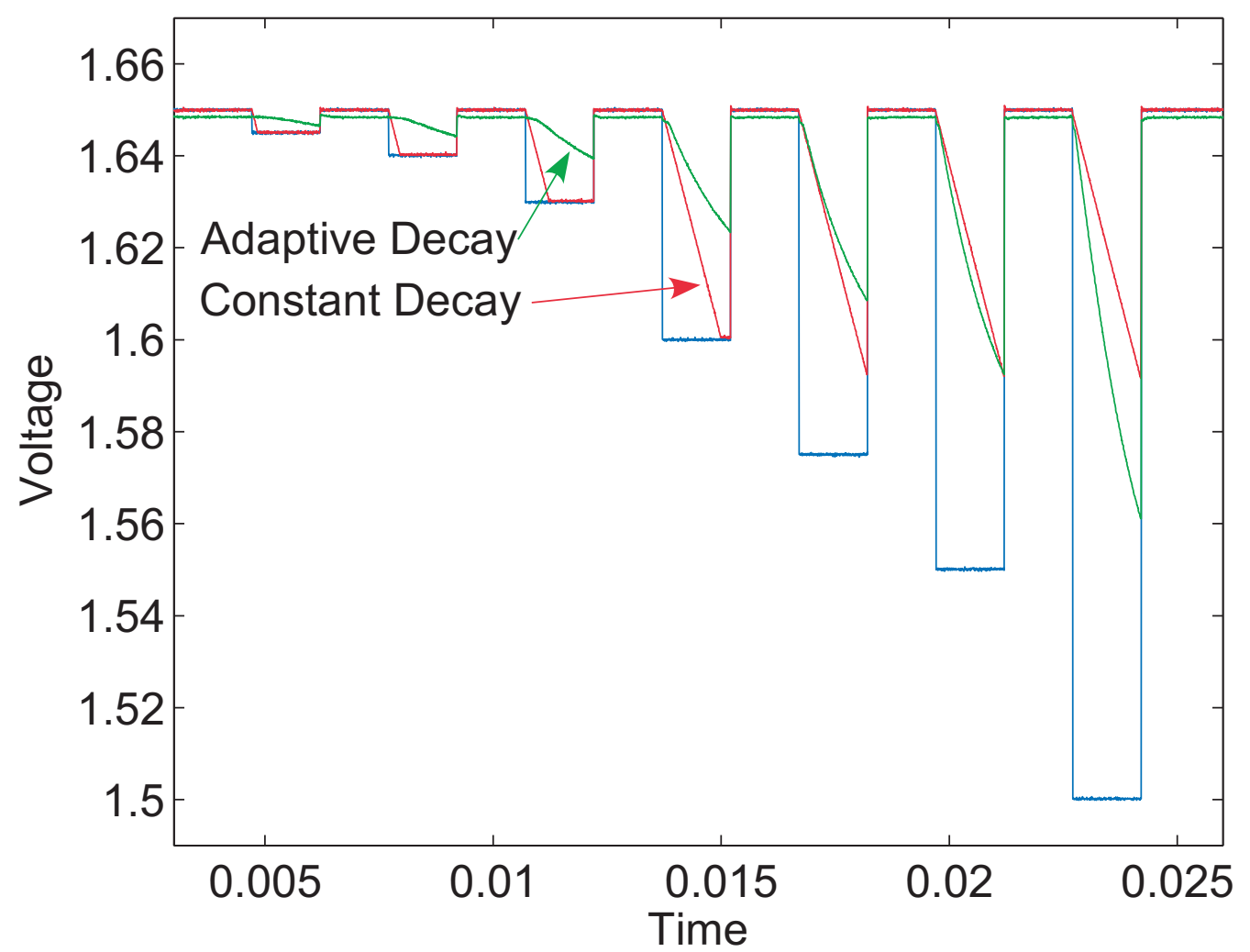

Figure 3.7: Comparing the constant decay and adaptive decay peak detectors

a very shallow slope. As the steps progressively increase the adaptive decay's slope gets steeper while the constant decay's slope remains constant.

\subsection{Nonlinear Filter}

While developing the magnitude circuit, the need for some kind of post peak detector filtering became evident. The problem (illustrated in fig. 3.8) is that if the peak detector's decay is slow enough for tolerable ripple, it is unresponsive to decreases in amplitude. A firstorder low-pass filter is not sufficient but a higher-order filter requires more area and power. Our novel solution is a follower-integrator with a nonlinear transconductance element which exhibits a soft-thresholding characteristic. This nonlinear filter's corner frequency increases exponentially with amplitude. As a result, small ripple is filtered with a low corner frequency 


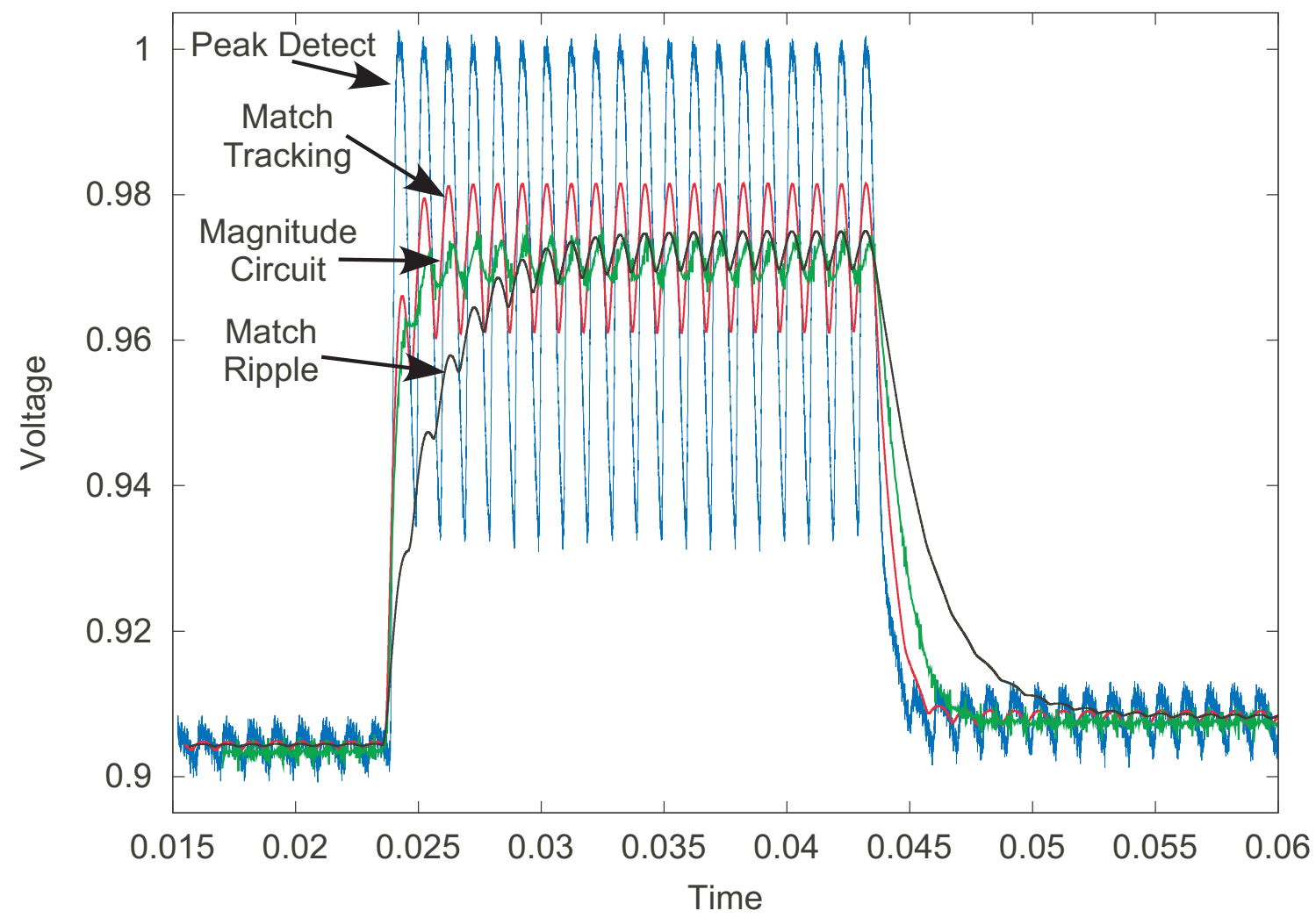

Figure 3.8: Comparison between nonlinear filter and first-order filters. "Match Ripple" is a filter with a low enough corner frequency to smooth the ripple but it takes longer to recover from large amplitude jumps. "Match Tracking" has a high enough corner frequency to follow at the same rate as the nonlinear filter, but it has about three times more ripple.

while sudden onsets are followed with a higher corner frequency.

\subsubsection{Nonlinear Transconductor}

The nonlinear transconductance element used in the nonlinear filter is depicted in fig. 3.9(a). The bump transistors [19] $\left(M_{5}\right.$ and $\left.M_{6}\right)$ have large $W / L$ (width-to-length) ratio, which when combined with the cross-coupled source degeneration results in lower transconductance at the origin. It's voltage-current characteristics are described by

$$
I_{\text {out }}=I_{B} \frac{\sinh \left(2\left(V^{+}-V^{-}\right) / V_{L}\right)}{1+w / 2+\cosh \left(2\left(V^{+}-V^{-}\right) / V_{L}\right)}
$$

where $V_{L}$ is the "linear" range of the OTA and $w$ is the ratio of the bump transistors' $W / L$ to the input pair's $W / L[14]$. This transconductance is shown in fig. 3.9(b), along with curve 


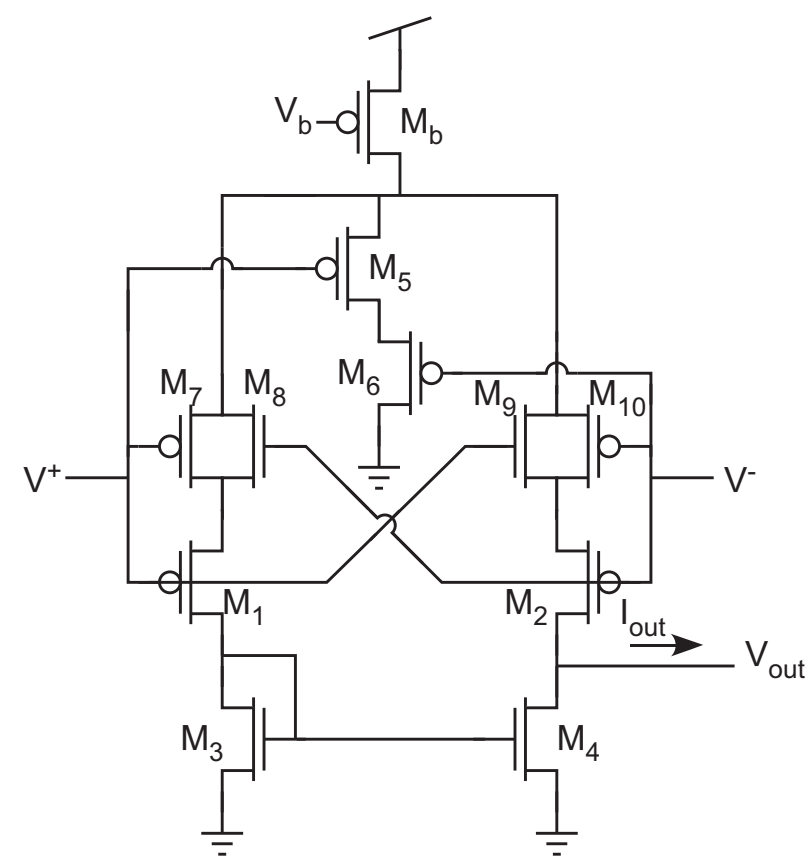

(a)

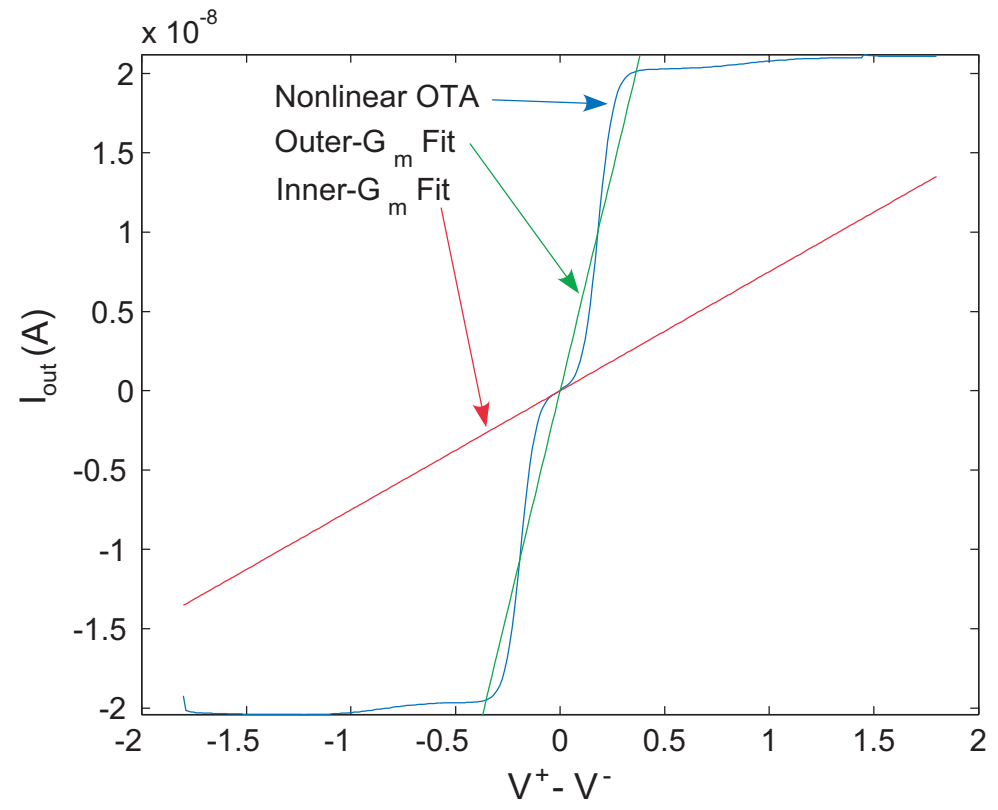

(b)

Figure 3.9: (a) The nonlinear OTA is a pFET based OTA with bump de-linearization and cross-coupled source degeneration. The bump transistors are made large to create a lower transconductance region at the origin. (b) $I_{\text {out }}$ for the nonlinear OTA. Included is a curve fit of the transconductance at the origin and a curve fit of the transconductance across the effective linear range. 


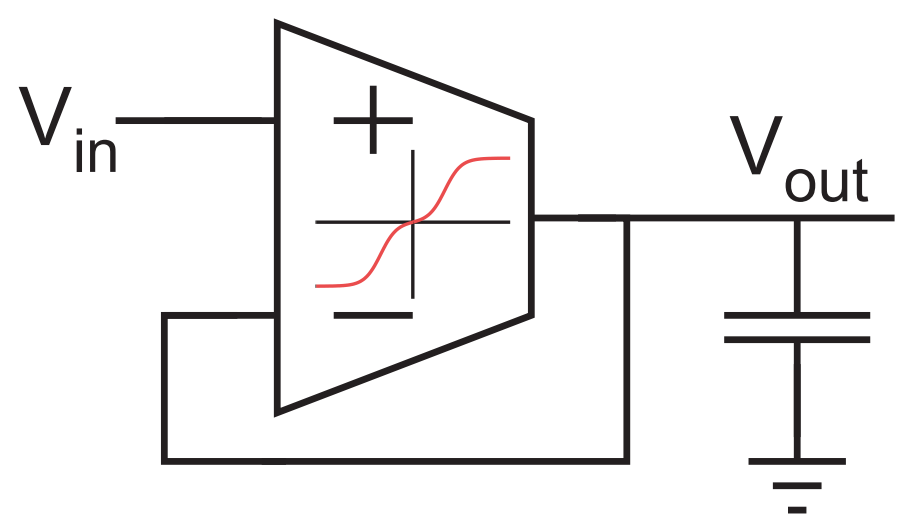

Figure 3.10: The nonlinear filter is a follower integrator with the nonlinear OTA used for the follower.

fits for the transconductance at the origin $\left(G_{m, \text { inner }}\right)$ as well as a curve fit over the effective linear range of the OTA $\left(G_{m, o u t e r}\right)$.

\subsubsection{Filter}

The filter in the second stage of the magnitude detector uses the nonlinear OTA in fig. 3.9(a) in a follower integrator configuration (fig. 3.10). This filter has a corner frequency which increases exponentially with amplitude as seen in fig. 3.11. The frequency response is plotted with the $\mathrm{AC}$ response of first-order filters with transconductances from the $G_{m}$ curve fits from fig. 3.9(b). Notice that for the smallest and largest amplitudes, the response of the nonlinear filter has the same corner frequencies as a filter with the smallest and largest transconductances from the OTA I-V plot.

The step response of the filter (fig. 3.12, includes responses of filters with curve fit $G_{m}$ 's from fig. 3.9(b)) gives more intuition of its operation. For a small step the nonlinear filter has the same response as the $G_{m, \text { inner }}$ fit. For a large step the nonlinear filter starts with the same time constant as $G_{m, o u t e r}$. As it approaches it's final value the time constant increases.

In the next section the nonlinear filter is combined with the peak detector of the previous section to smooth ripple while still tracking large changes. 


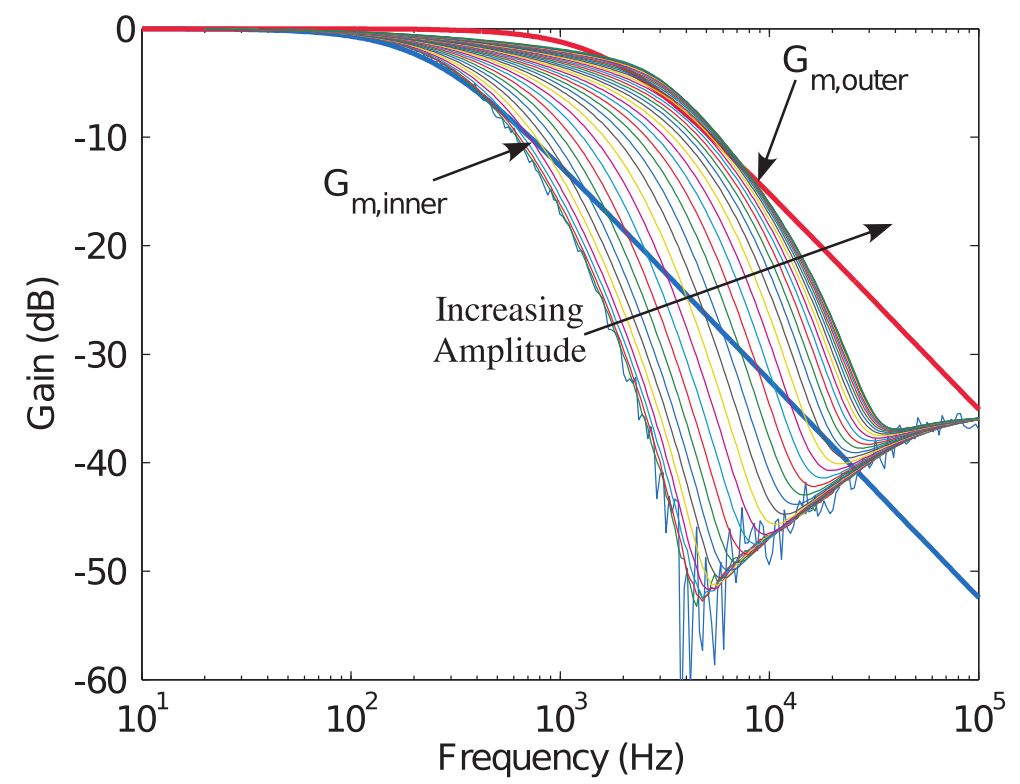

(a)

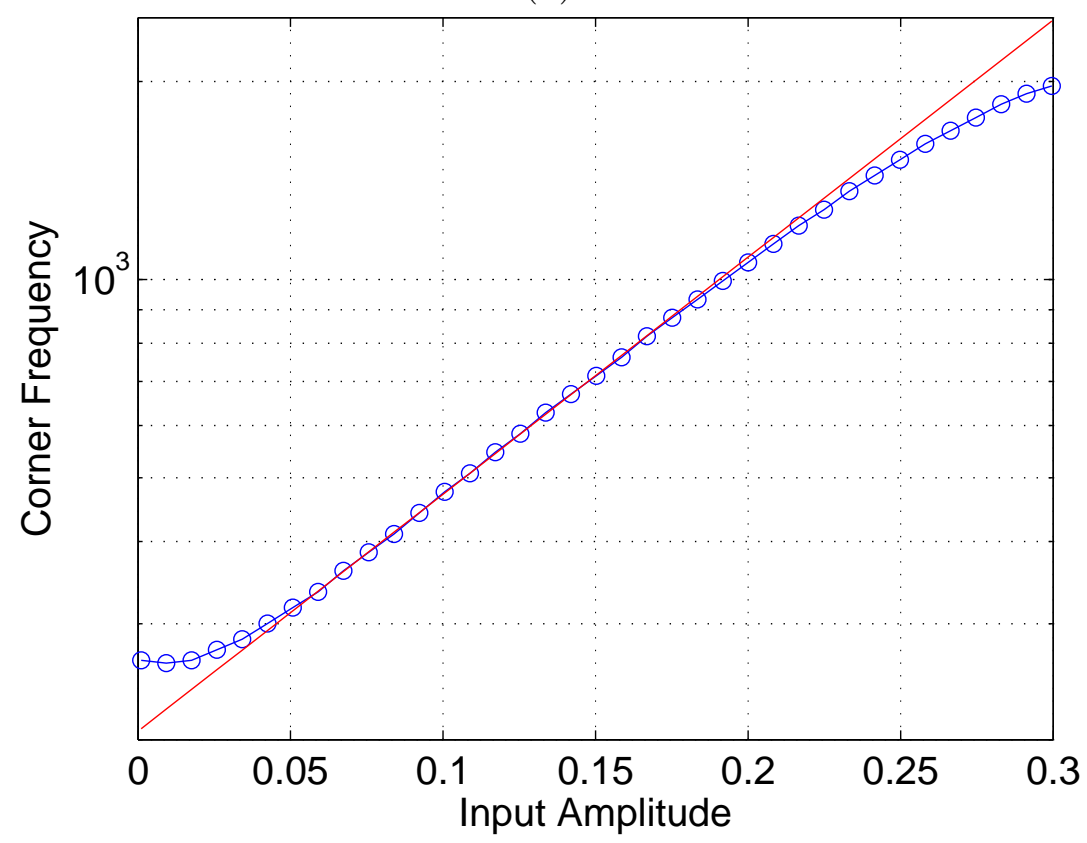

(b)

Figure 3.11: (a) Frequency response of the nonlinear filter for increasing amplitude. The frequency response for the smallest amplitude lines up with the corner frequency of a follower integrator with $G_{m}=G_{m, \text { inner }}$ and the response for the largest amplitude lines up with the corner frequency of a follower integrator with $G_{m}=G_{m, \text { outer }}$. (b) Corner frequency as a function of amplitude. From $40 m V_{p k}$ to $200 m V_{p k}$ the corner frequency is exponentially dependent on amplitude. 


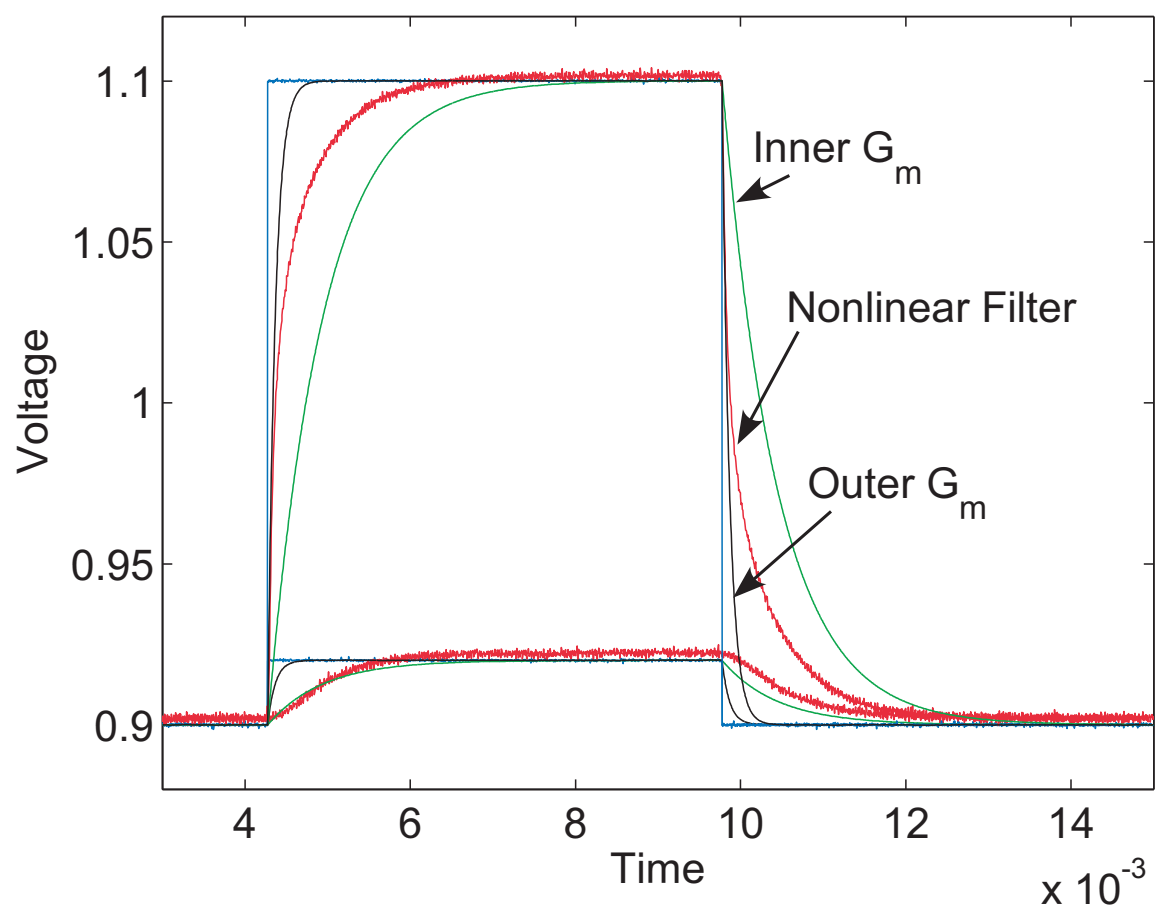

Figure 3.12: Step response of the nonlinear filter. For a small step the nonlinear filter stays in its inner linear range and responds with the same time constant as the inner $G_{m}$ fit. For the large step the nonlinear filter initially tracks with the same time constant as the outer $G_{m}$ fit, but its time constant decreases as it approaches the final value.

\subsection{Full Magnitude Circuit}

Figure 3.13(a) is the complete magnitude circuit. Figure 3.13(b) demonstrates the operation of the magnitude circuit. The input is a sine wave which steps from a $5 m V_{p k}$ amplitude up to a $100 m V_{p k}$ amplitude, then down to a $10 m V_{p k}$ amplitude. The peak detector stage rectifies the signal with large ripple, allowing it to track the large amplitude changes while the nonlinear filter follows behind cleaning up the ripple.

Figure 3.14 shows the dynamic range of the magnitude circuit. The dynamic range for the $2 k H z$ case is $68 d B$. The limit on smallest detectable signal is set by the noise floor which is $100 \mu \mathrm{V}$. The output referred noise is shown in fig. 3.15.

Since this circuit is intended for speech processing its response to speech waveforms is of particular concern. Figure 3.16 shows the magnitude circuit's response to an unfiltered 


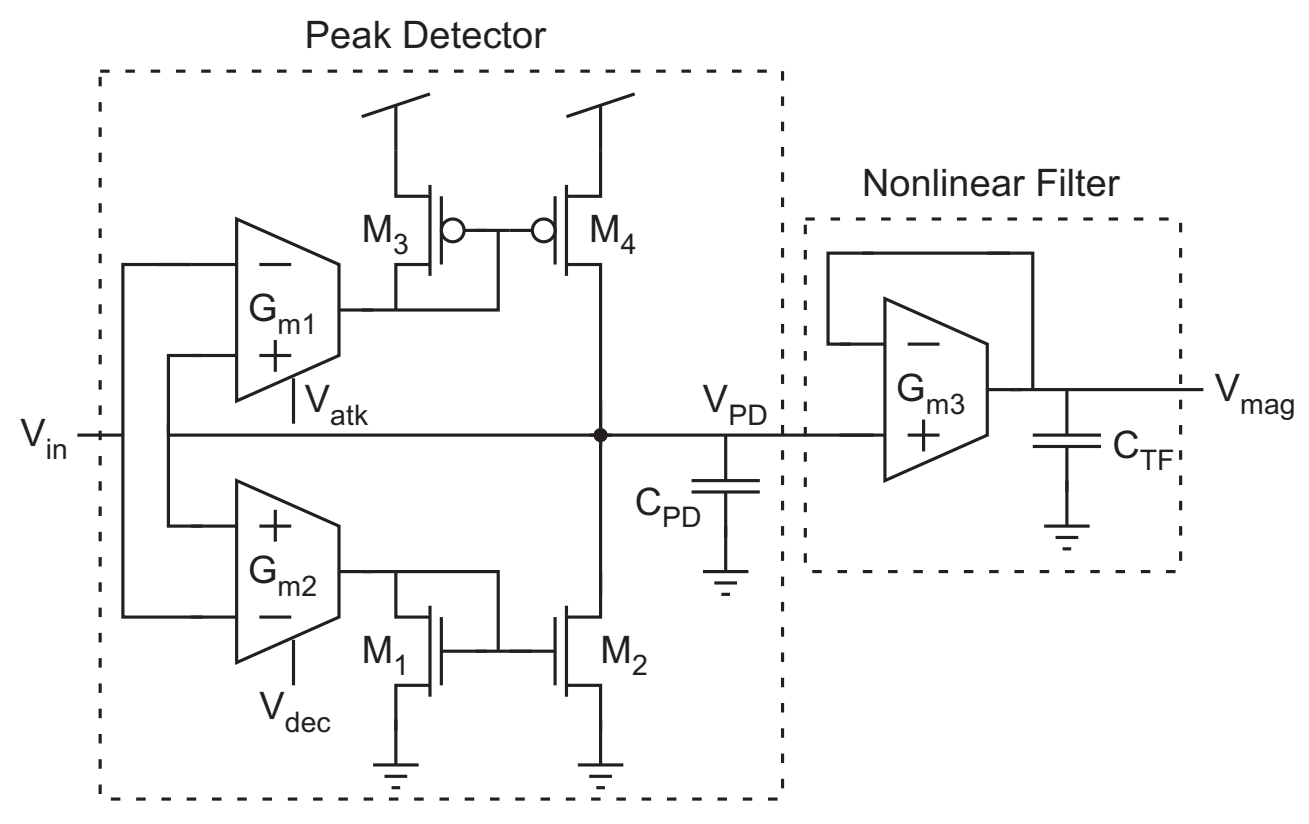

(a)

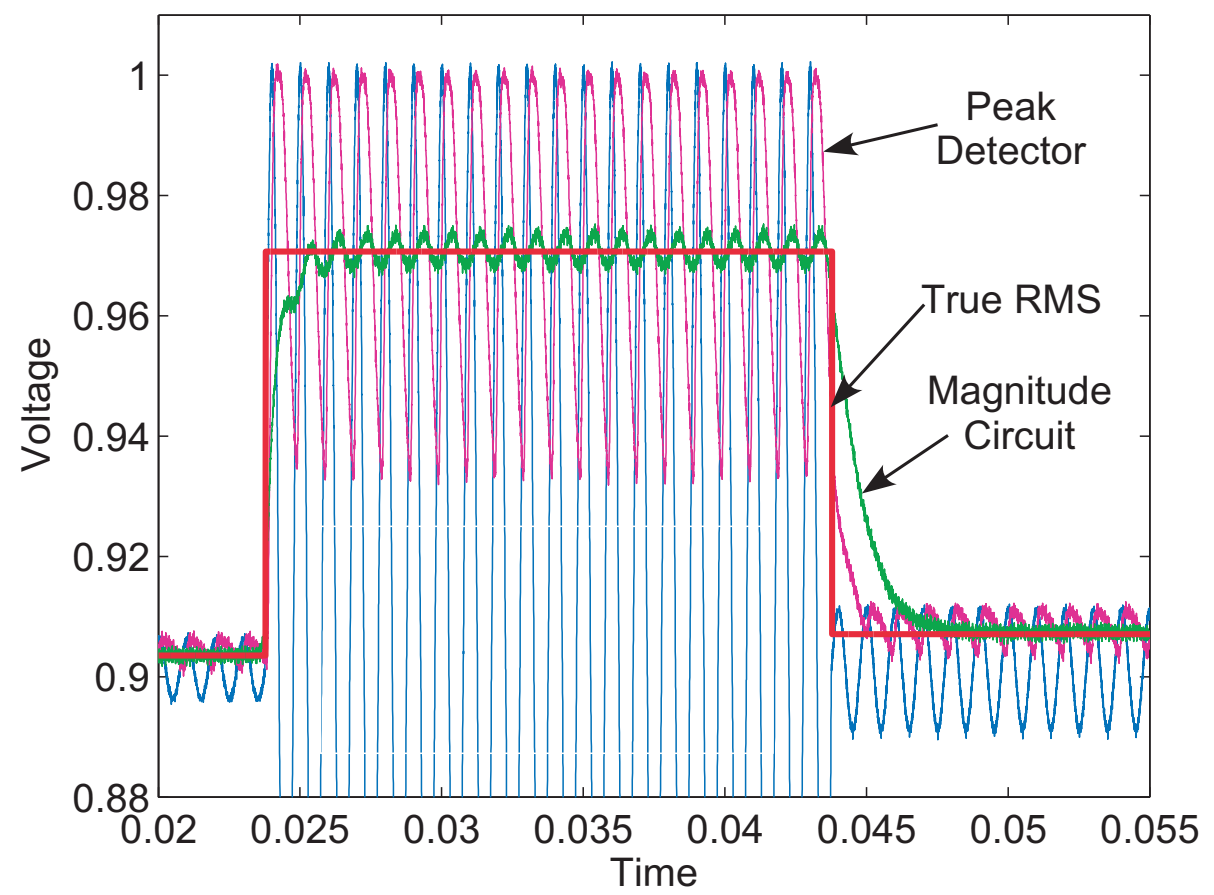

(b)

Figure 3.13: (a) Proposed magnitude detector. It consists of a peak detector with amplitude dependent decay rate and a nonlinear filter with amplitude dependent time constant. (b) Demonstration of each stage of the magnitude circuit. The peak detector tracks the RMS with large ripple and the nonlinear filter follows behind smoothing it out. 


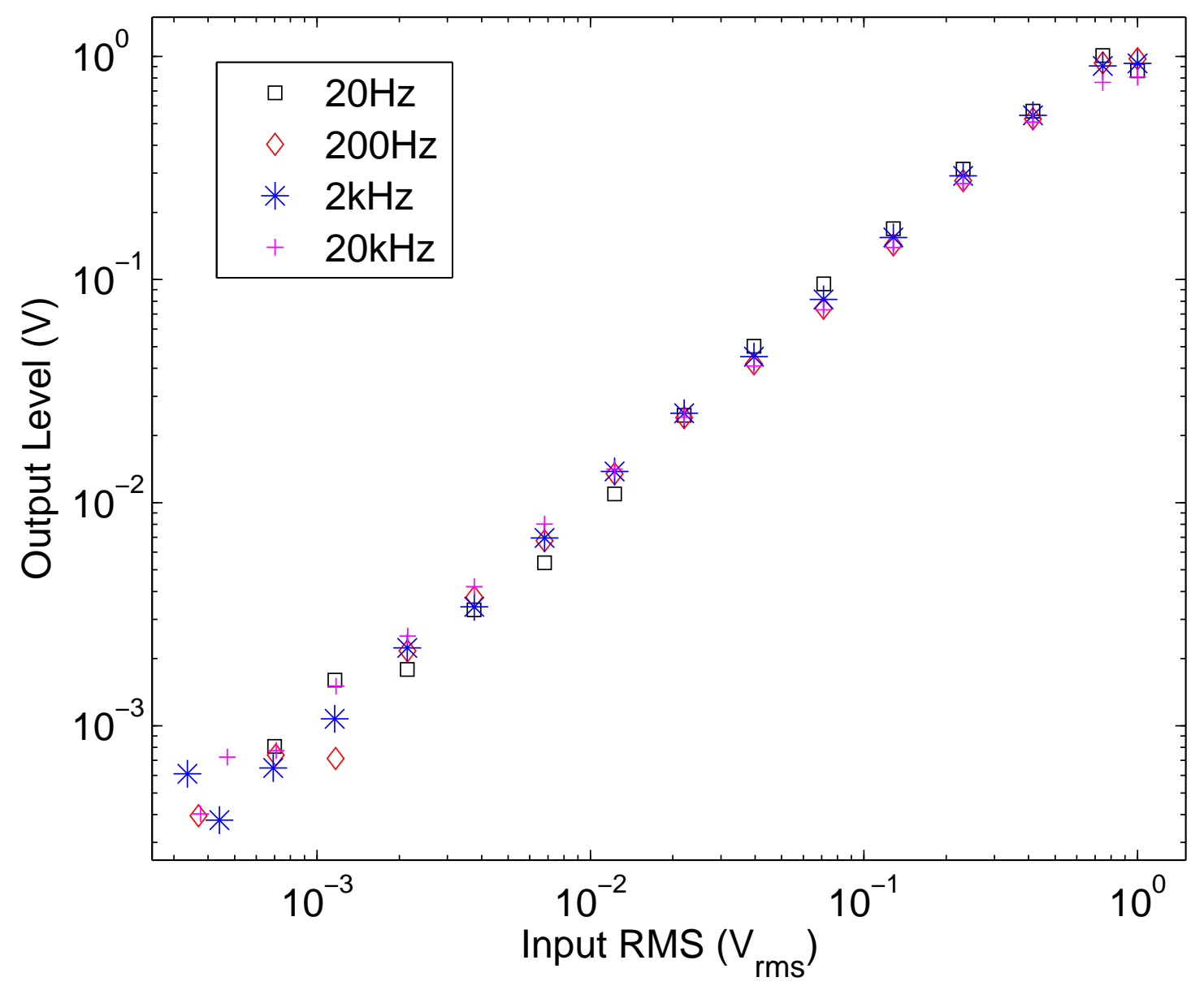

Figure 3.14: Dynamic range of the magnitude circuit. At $2 \mathrm{kHz}$ the circuit provides approximately $68 d B\left(400 \mu V_{r m s}\right.$ to $\left.1 V_{r m s}\right)$ of commensurate information.

speech segment. For comparison, fig. 3.16(a) includes the calculated ideal RMS of the waveform. The response of the magnitude detector matches the ideal RMS well. Figure 3.16(b) compares the response of our magnitude detector with the traditional peak detection technique.

\subsubsection{Tuning}

The magnitude detector presented in this chapter has three parameters to tune its performance: attack, decay, and smooth. These are all controlled by the bias currents in the three OTA's in the circuit. Having these three tunable parameters makes this circuit great for use in systems which use floating-gate biases for programmability. This section presents 


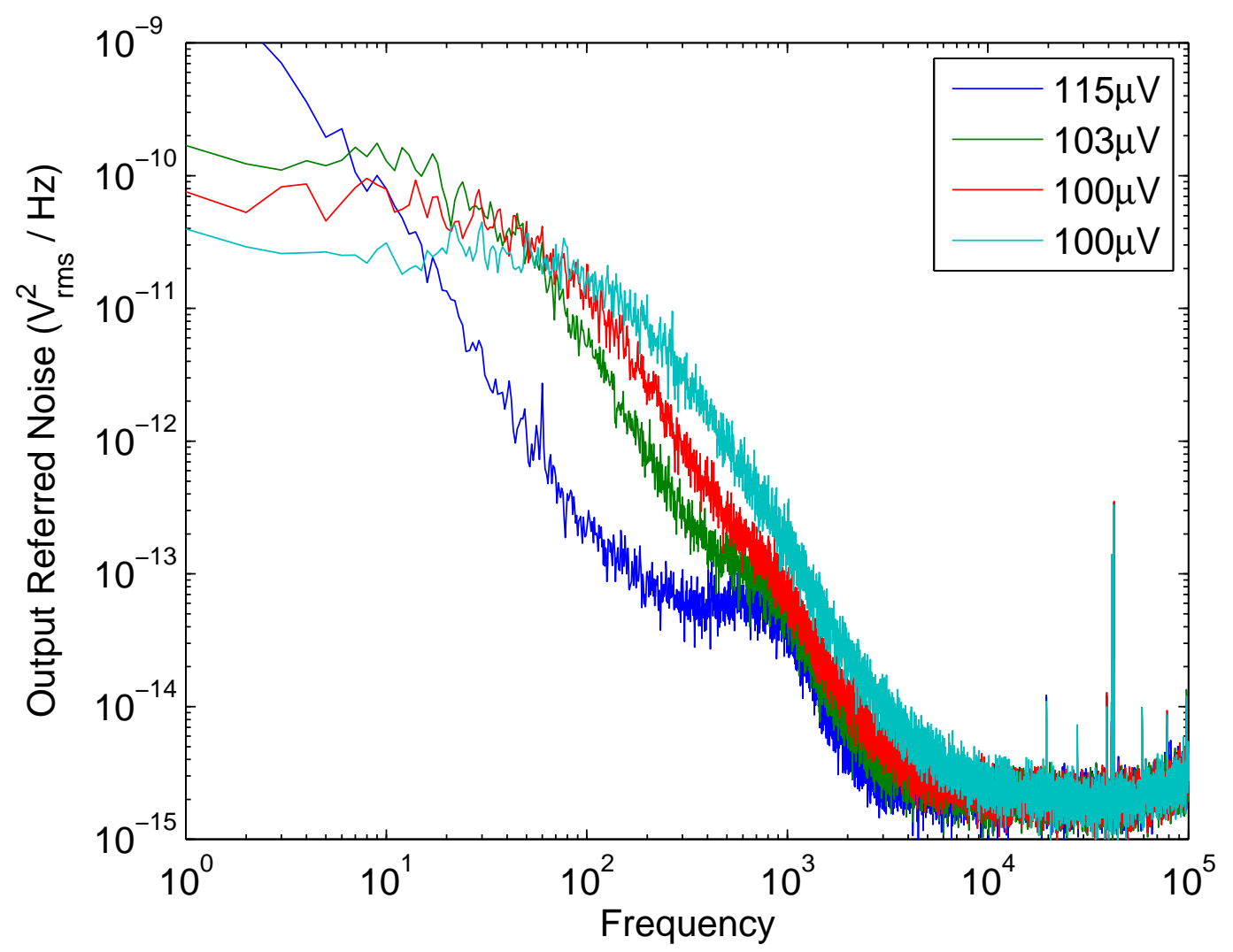

Figure 3.15: Output referred noise for the magnitude circuit. The integrated noise level of approximately $100 \mu \mathrm{V}$ sets a floor on the dynamic range.

an initial programming algorithm which is depicted in fig. 3.17.

The input used in the tuning process is a sine wave at the frequency for the sub-band of operation. This sine wave has a linearly increasing amplitude. The tuning procedure begins with the circuit biased such that it is functional. Then the decay is adjusted such that the slope matches the ideal RMS. Once the desired slope is achieved, the attack is adjusted to null out any offset. The attack and decay are not independent so it may be necessary to iterate on these steps until the performance is within tolerance. After the attack and decay are set, the nonlinear filter can be programmed for an appropriate compromise between tracking and ripple. 


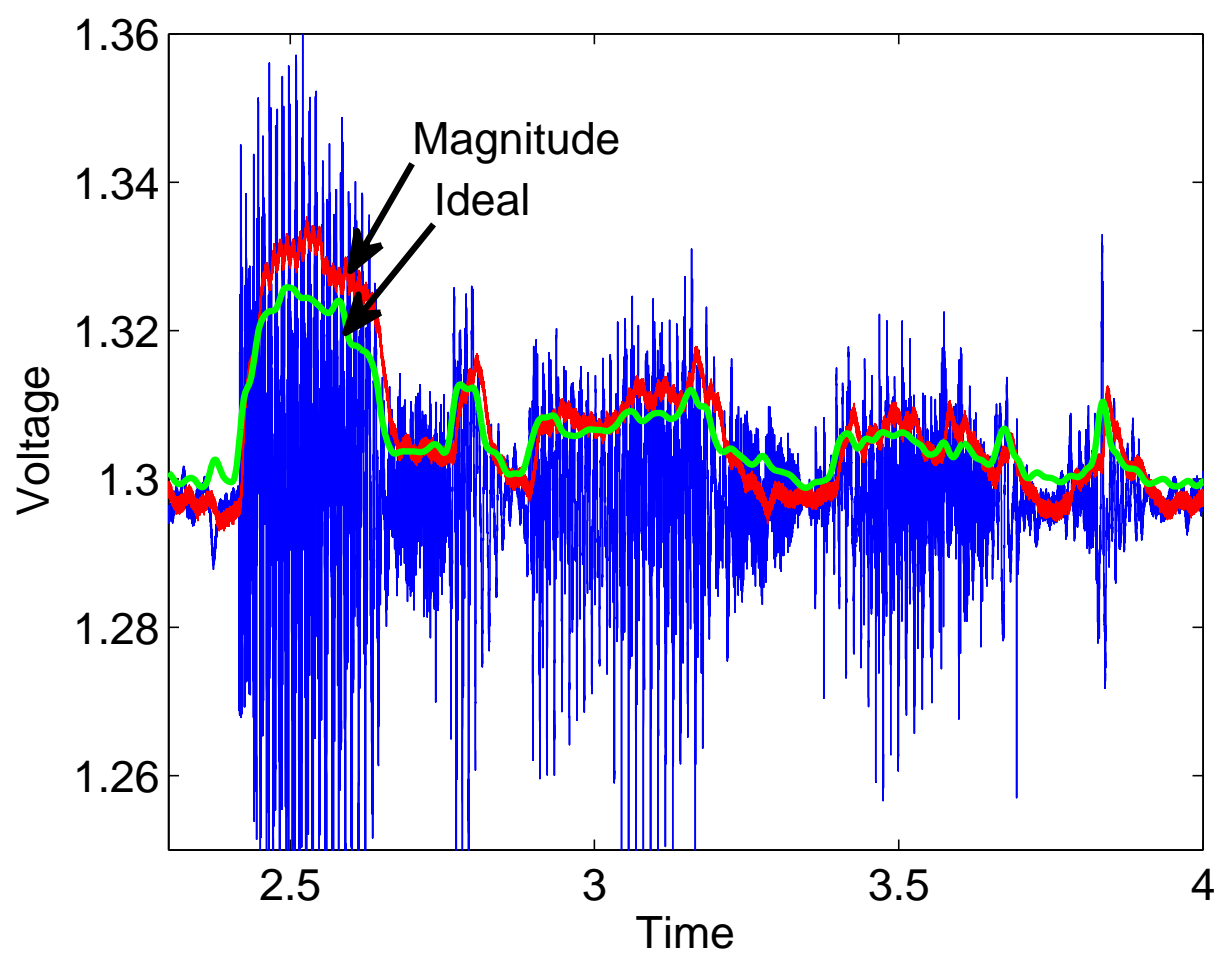

(a)

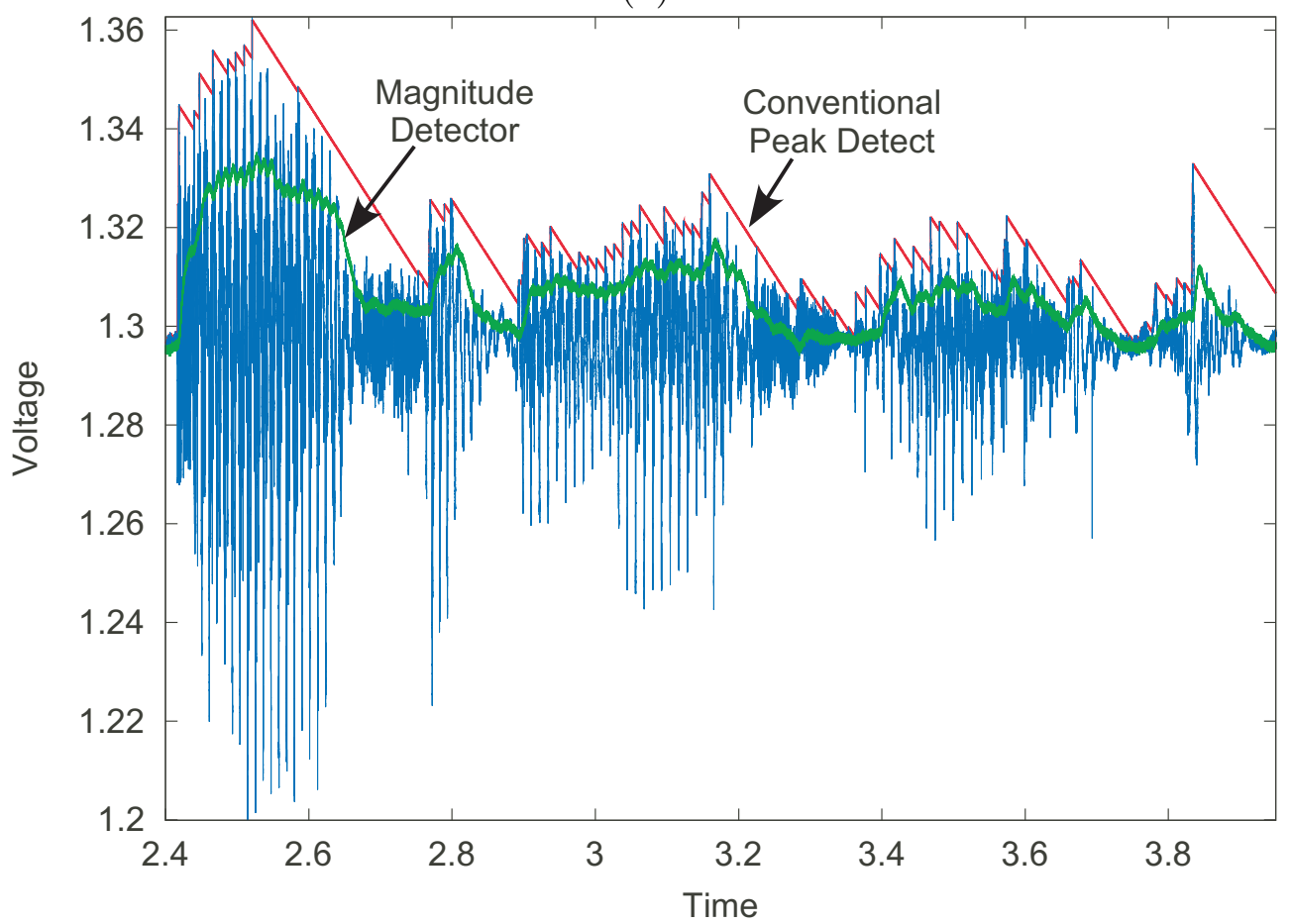

(b)

Figure 3.16: Magnitude circuit tracking the RMS of an unfiltered speech segment 


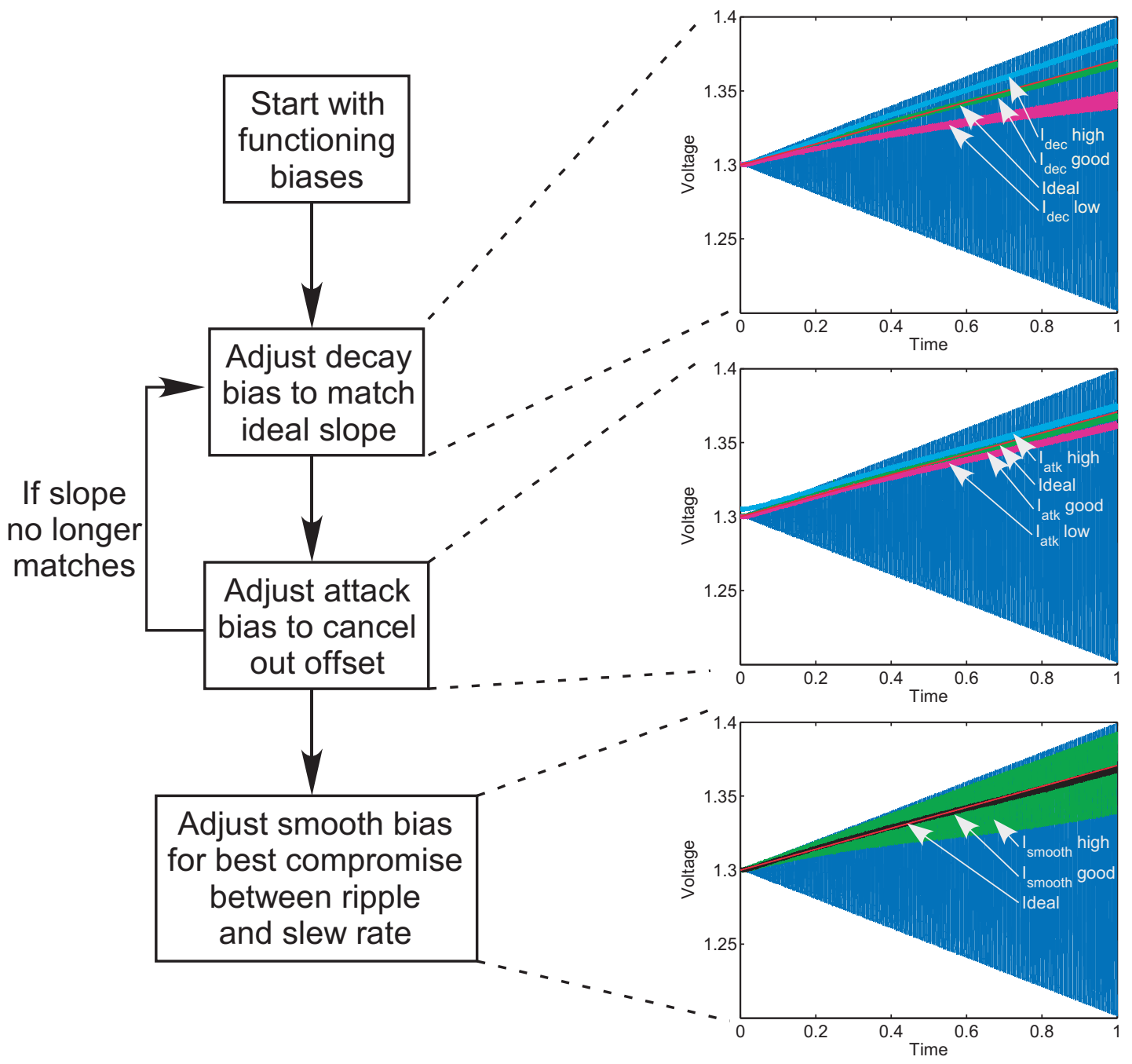

Figure 3.17: Tuning algorithm for the magnitude circuit. The input used is a sine wave at the desired operation frequency, with a linearly increasing amplitude. First the circuit is biased such that it is functional. Then the decay is adjusted such that the slope matches the ideal RMS. Once the desired slope is achieved, the attack is adjusted to null out any offset. The attack and decay are not independent so it may be necessary to repeat these steps until the performance is within tolerance. After the attack and decay are set, the nonlinear filter can be programmed for an appropriate compromise between tracking and ripple. 


\section{Chapter 4}

\section{Analog Frequency Decomposition}

In this chapter we combine the bandpass filter (chapter 2) with the magnitude detector (chapter 3) to form a spectral decomposition system. The first section deals with the $0.5 \mu m$ event-detection filter bank which has 8 channels and a maximum quality factor of 5 . The second section deals with the $0.18 \mu \mathrm{m}$ filter bank, which has 16 channels and a maximum quality factor of 3 .

\subsection{Event-detection Filter bank}

The event-detection system (discussed in chapter 6), was fabricated on a $0.5 \mu \mathrm{m}$ process and measures approximately $1.5 \mathrm{~mm}$ by $1.5 \mathrm{~mm}$ (see die photograph in fig. 6.3). There are eight frequency channels, and the biases are set by on-chip resistive dividers. The filter bank can be tuned to a maximum quality factor of 5, and a maximum gain of $18 \mathrm{~dB}$ (fig. 4.1). In this section the filter bank is used with the channels spanning $100 \mathrm{~Hz}$ to $2 \mathrm{khz}$ with logarithmic spacing.

Figure 4.2 shows the step response of the filter bank for $\mathrm{Q}=3$ and $\mathrm{Q}=5$. Since the $\mathrm{C}^{4}$ has negative gain and the magnitude half wave rectifies the signal, the system is slow to respond to the positive step. The difference in resonance between the $\mathrm{Q}=3$ and $\mathrm{Q}=5$ cases can be clearly see when comparing figs. $4.2(\mathrm{~b}) \&(\mathrm{~d})$. As expected, we observe that the step response of higher frequency channels in these constant-Q filter banks, is simply a dilation of the step response of lower frequency channels. 


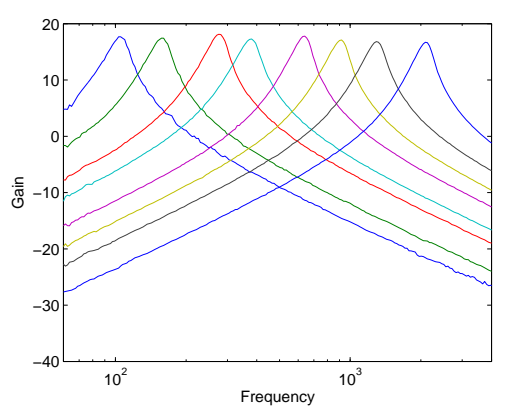

(a)

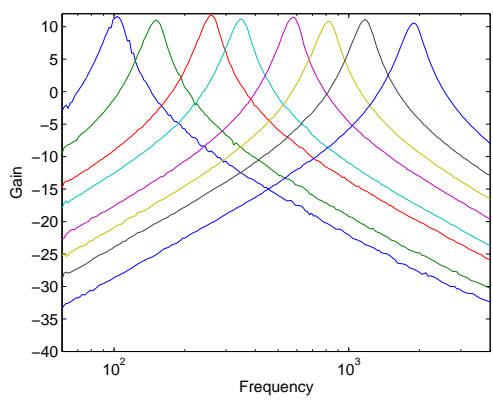

(b)

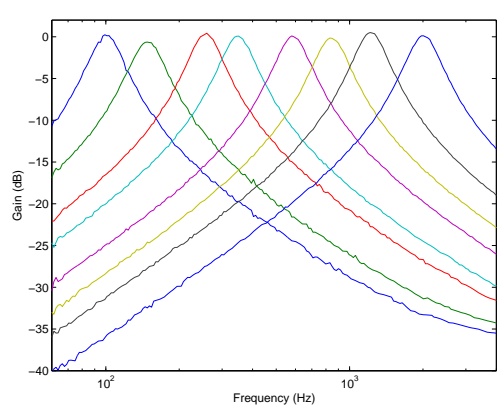

(c)
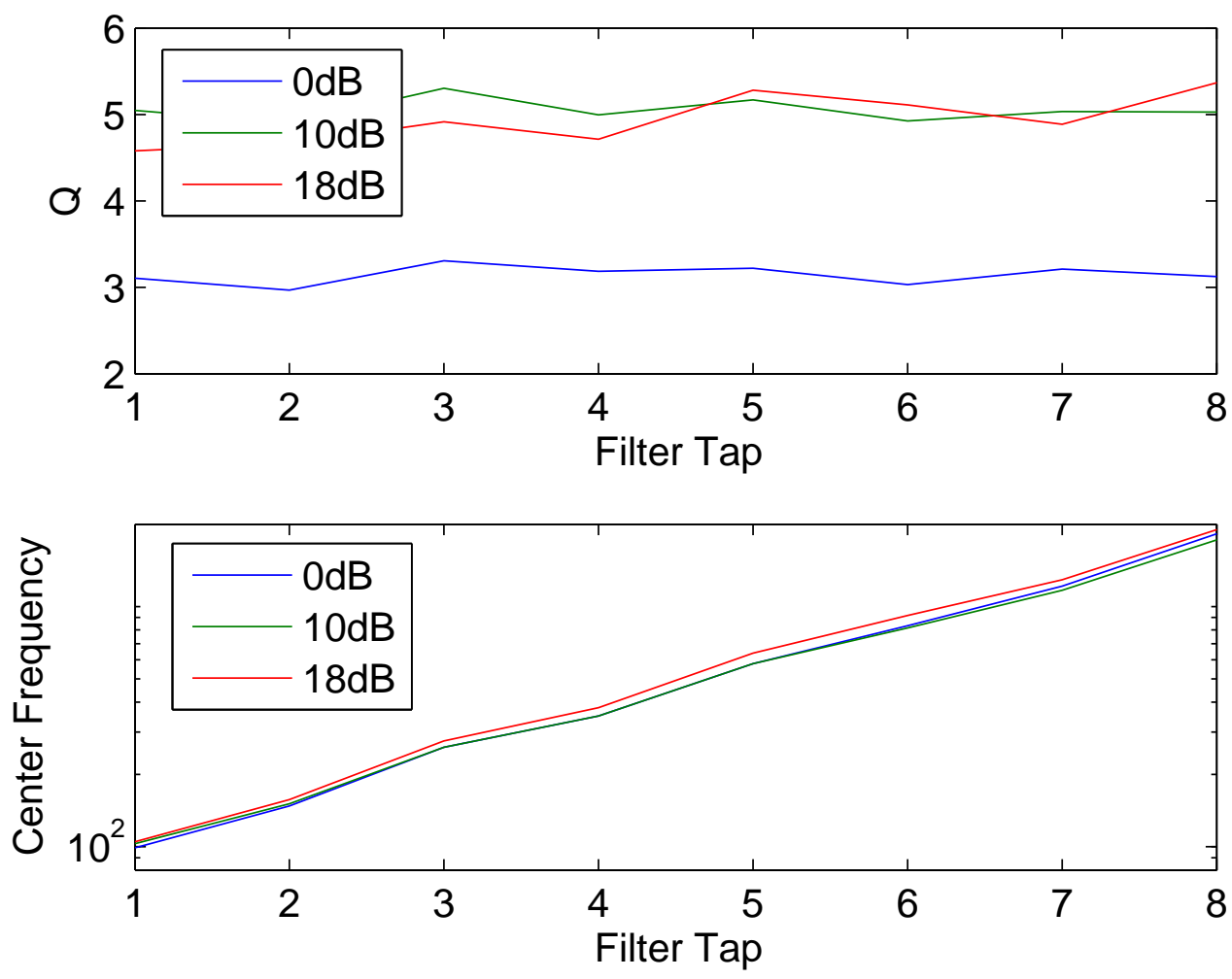

(d)

Figure 4.1: $A C$ response of the event-detection filter bank. (a) Gain=18dB, $Q=5$ (b) Gain $=12 \mathrm{~dB}, \mathrm{Q}=5$ (c) Gain=0dB, $\mathrm{Q}=3$ (d) $\mathrm{Q}$ and center frequency as a function of filter tap 

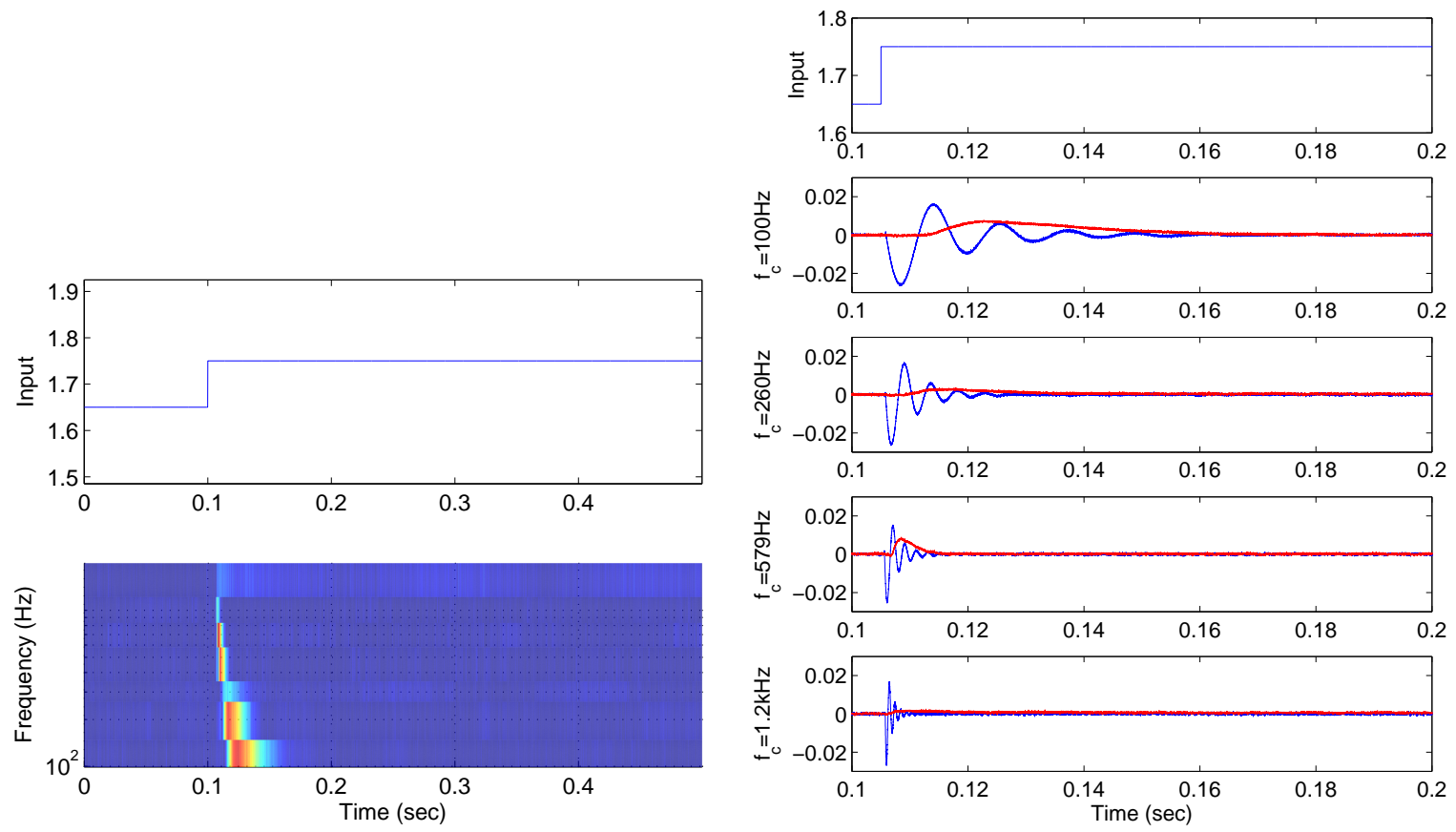

(a)

(b)
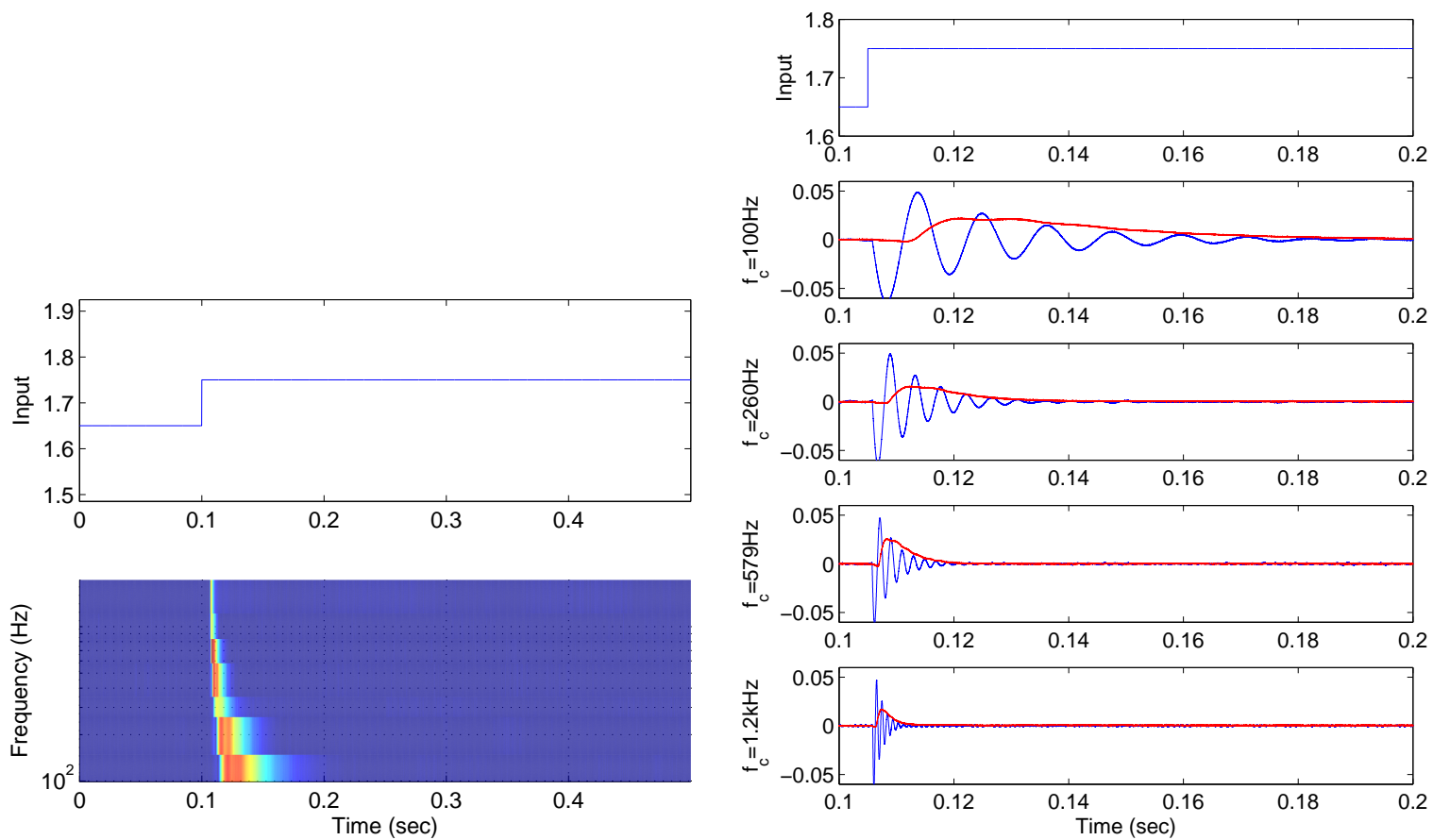

(c)

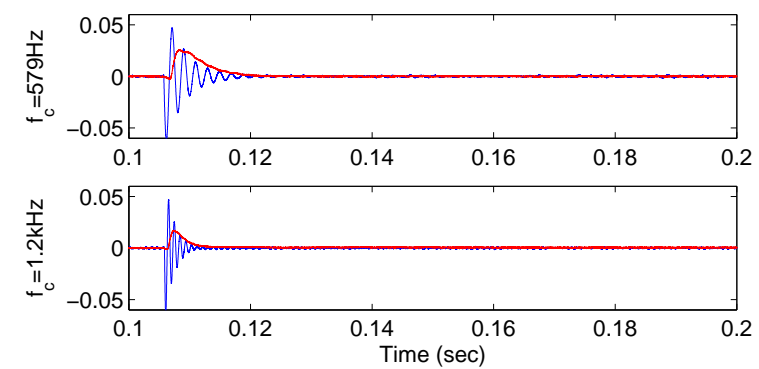

(d)

Figure 4.2: Filter response in blue and magnitude response in red. (a) Step response spectrogram. $Q=3$ (b) Step response sub-bands. $Q=3$ (c) Step response spectrogram. $Q=5$ (d) Step response sub-bands. $\mathrm{Q}=5$ 

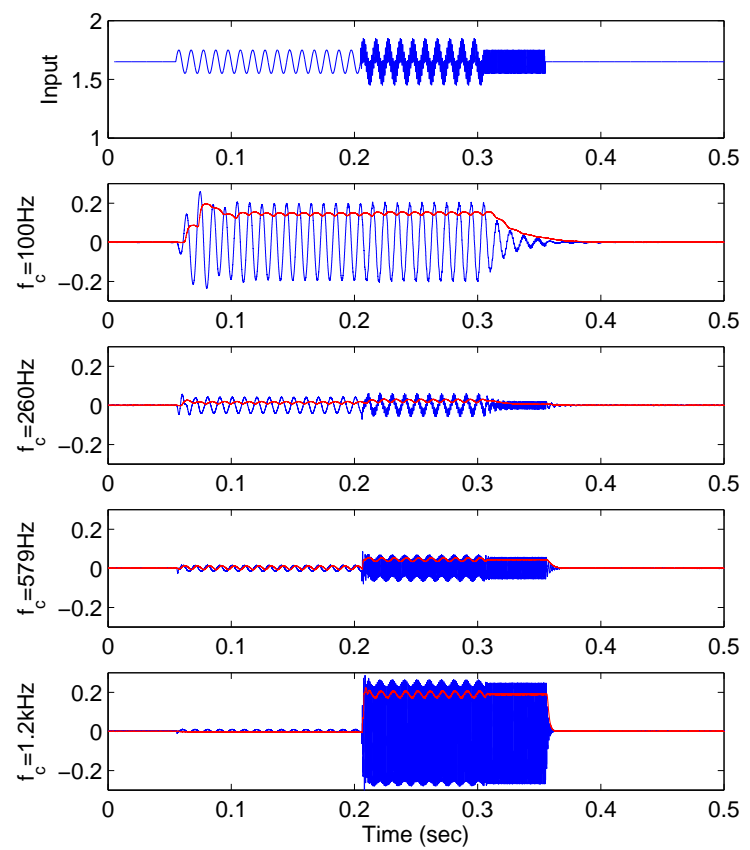

(a)
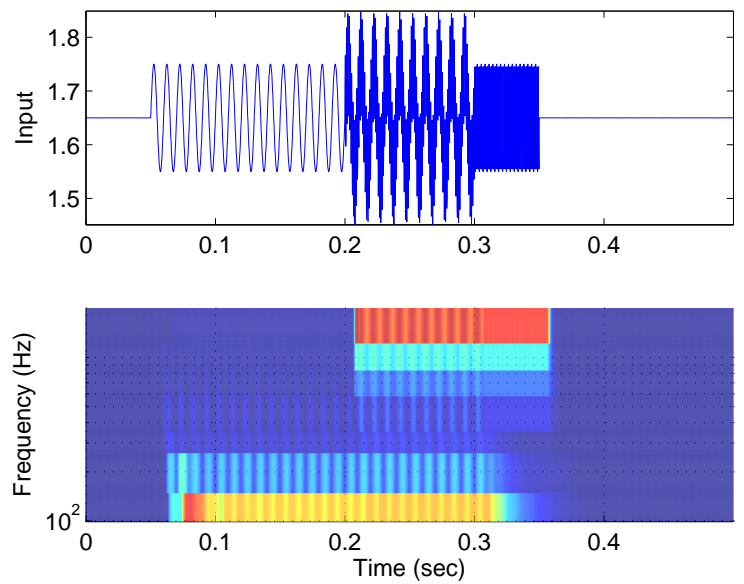

(b)

Figure 4.3: Decomposition of $100 \mathrm{~Hz}$ and $1 \mathrm{kHz}$ sine waves.

Figure 4.3 demonstrates the decomposition of two sine waves $(100 \mathrm{~Hz}$ and $1 \mathrm{kHz})$ which overlap in time but not in frequency. Due to the relatively low quality factor $(\mathrm{Q}=5)$ there is noticeable spectral leakage into adjacent bands. However, in this example we can still distinguish between the two tones.

\section{$4.2 \quad 0.18 \mu m$ Filter bank}

The $0.18 \mu \mathrm{m}$ front-end (system diagram shown in fig. 1.3) has 16 channels which are programmed with floating-gate transistors (layout of full chip shown in fig. 4.7). At the time of this writing, we do not possess the resources required to program the floating-gate transistors (a circuit board with the necessary infrastructure is currently being designed), so the results shown in this section were obtained with a test-column included on the chip. The test-column is the same as the channels included in the system, except the floatinggate biases are by-passed and digital-to-analog converters are used to set bias voltages. The biasing of the filter bank is shown in fig. 4.4. The passband gain is $2.5 \mathrm{~dB}$, the $\mathrm{Q}$ is 2.8 , and 


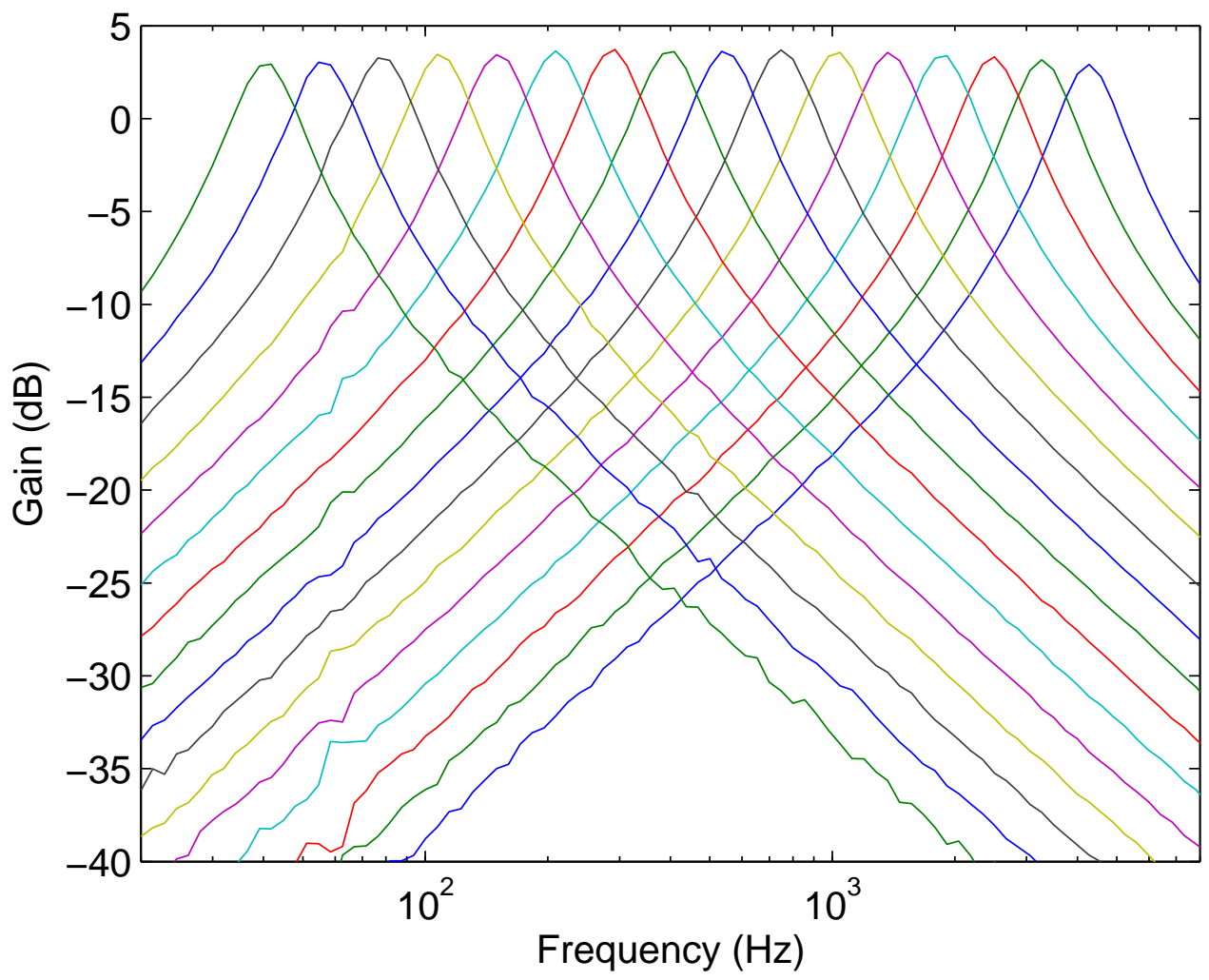

Figure 4.4: AC response of $0.18 \mu m$ filter bank used in this chapter

the filters are spaced from $40 \mathrm{~Hz}$ to $4 \mathrm{kHz}$. The combined power consumption of the filters and magnitude detectors for all 16 channels is $4 \mu W$

Figure 4.5 shows a spectrogram generated from the output of the magnitude in each subband. The filter bank naturally gives the response on a log frequency axis. For reference, the spectrogram is also shown with a linear frequency axis.

Figure 4.6 shows the response of individual sub-bands to the phrase "needless to say". By inspection, we can see that the $107 \mathrm{~Hz}$ channel contains the fundamental frequency for the voiced sounds, while the $209 \mathrm{~Hz}$ and $292 \mathrm{~Hz}$ channels contain the first two harmonics. The unvoiced sounds can be seen in the $4.3 \mathrm{kHz}$ channel. 

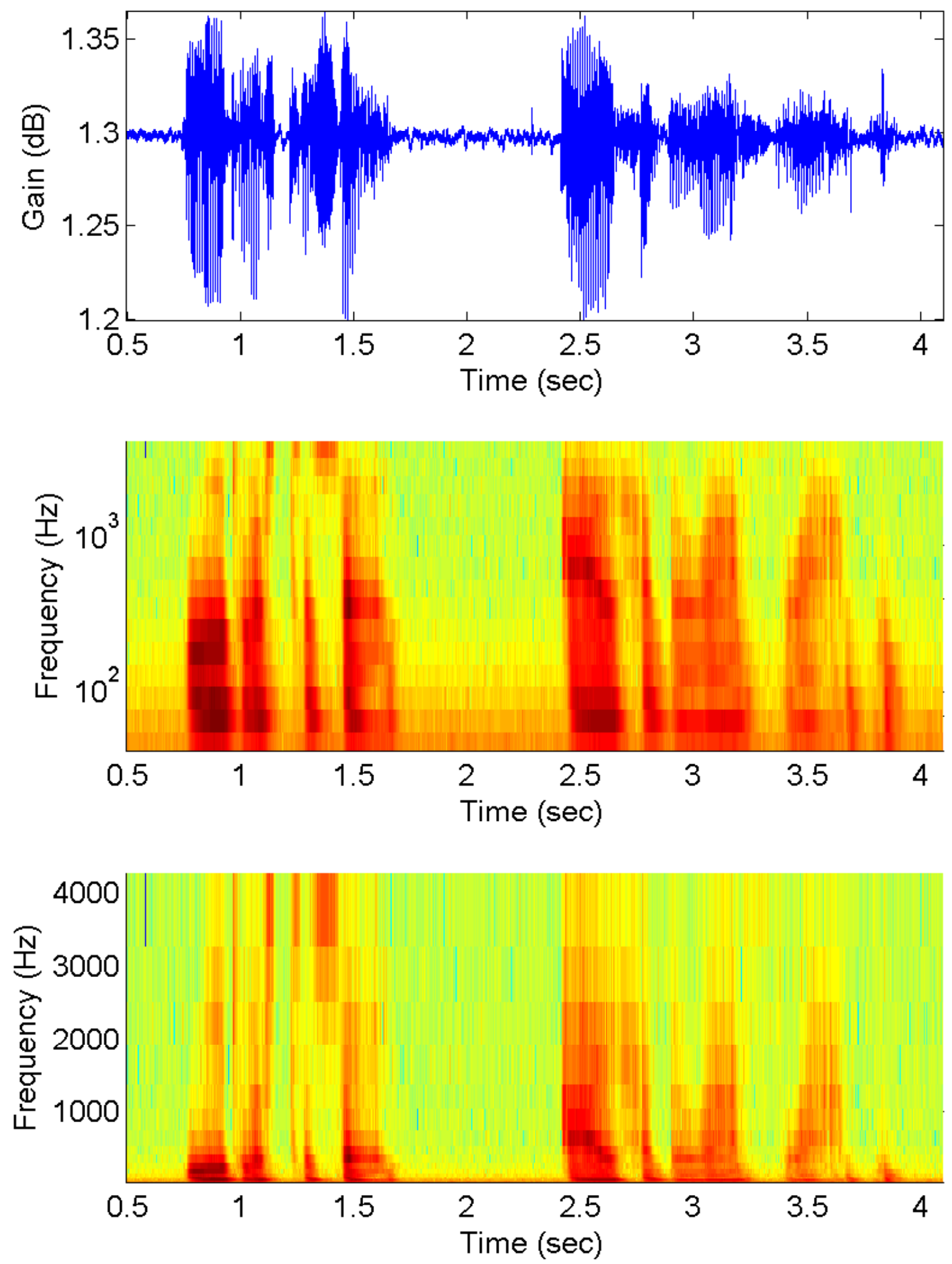

Figure 4.5: Spectrogram of speech signal with $0.18 \mu m$ filter bank. Both log and linear frequency axes are shown. 

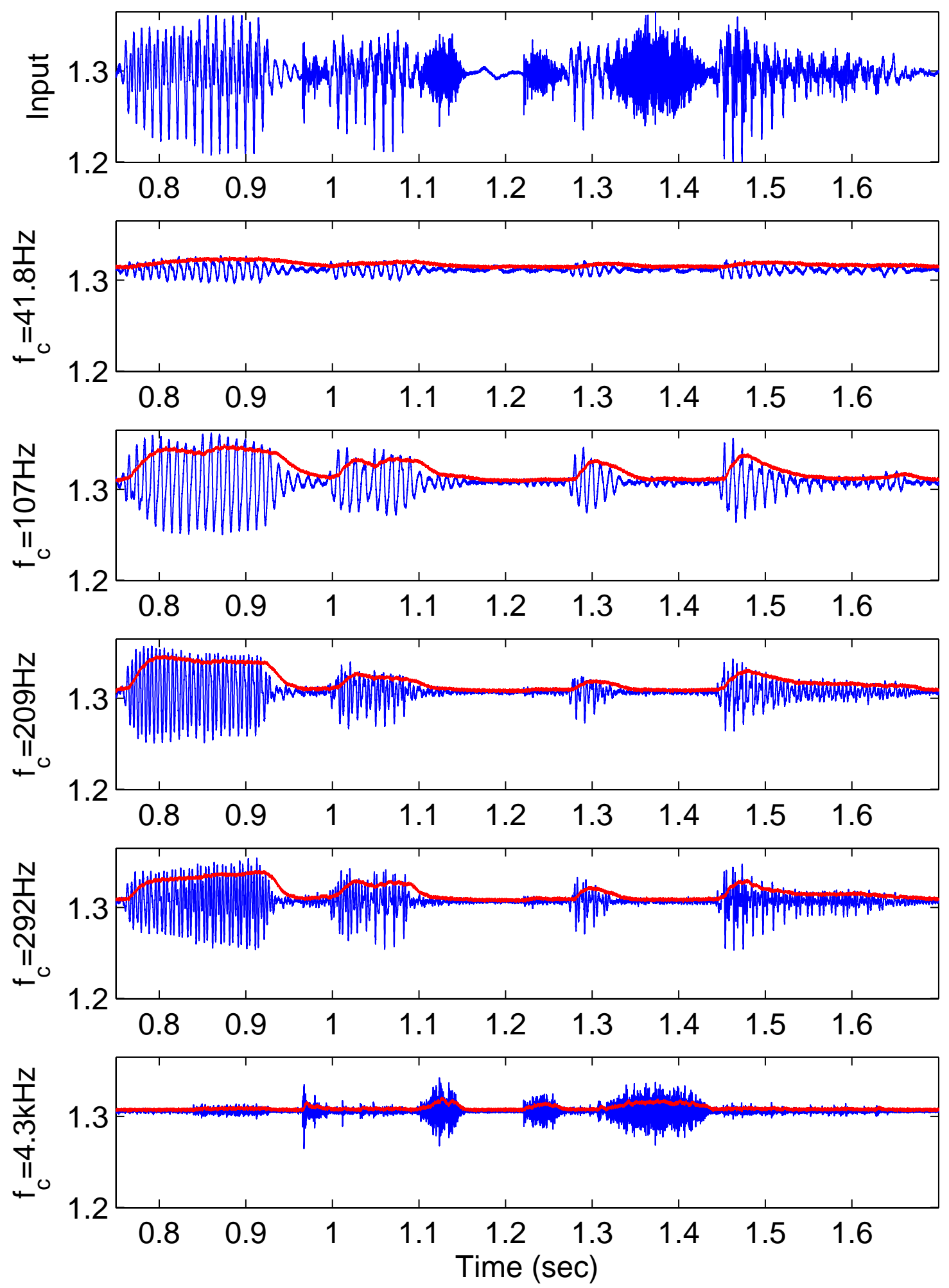

Figure 4.6: $0.18 \mu m$ filter bank. Decomposition of speech signal. Filter output in blue and magnitude output in red. 


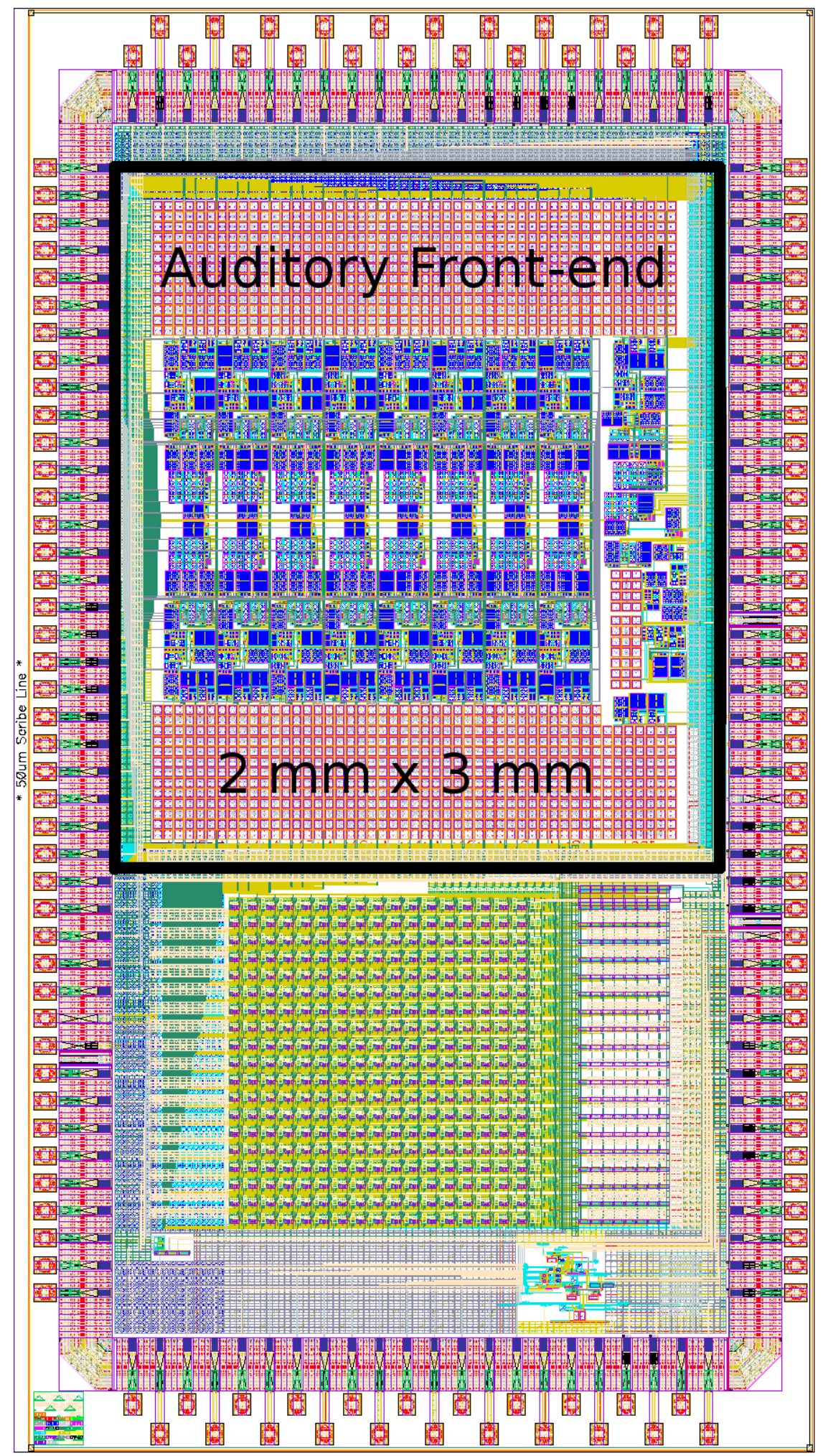

Figure 4.7: Layout of the $0.18 \mu m$ auditory front-end 


\section{Chapter 5}

\section{Lateral Coupling in Cochlear Models}

Many researchers have looked to biology for inspiration in the design of sensory systems. Biological processing is low-power, highly parallel, fault-tolerant, and works well with noisy and incomplete information. One biological subsystem of interest to audio processing is the cochlea, and several circuits have been developed which model the cochlea, including $[20,21,22,23,24,25]$. The cochlea is the front-end of the biological auditory system. Accordingly, a circuit which accurately models the functionality of the cochlea will provide a low-power audio processing front-end. Such a front-end is well suited for audio-processing applications like speech recognition [26], noise suppression [27], or sound localization [28]. In addition, a low-power, biologically plausible, audio front-end is of interest for hearing aids and cochlear implants.

This section presents a novel silicon cochlea model which better accounts for the effect of local fluid coupling on the basilar membrane [29]. This fluid coupling is emulated by coupling an extra wideband filter to each narrowband filter in the array, as shown in Fig. 5.1, which in turn produces sharper high-frequency slopes, much like biology. In this section I present some of the biological background and give a short survey of earlier silicon cochlea models. I then briefly discuss the bandpass-filter element used in the circuit before presenting the silicon cochlea model along with measurements of the performance of a single filter tap. 


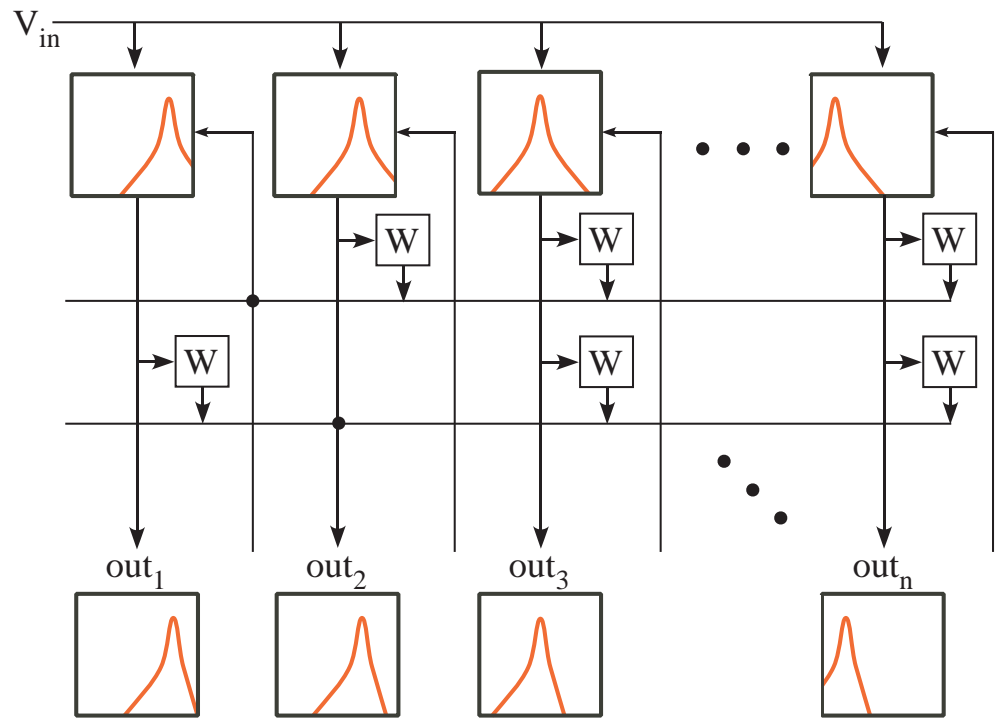

Figure 5.1: Lateral coupling of bandpass filters to achieve a frequency response similar to the cochlea. An array of bandpass filters have their outputs coupled to the input of the other filters. This effectively subtracts the response of the neighboring filters. By varying the weight of the connection between filters, the output can have the desired cochlea-inspired frequency response. In this case, the higher-frequency filters are weighted more than the lower-frequency filters, resulting in the asymmetrical frequency response seen in the figure.

\subsection{Biological Motivation}

The cochlea acts as the audio front-end in the biological auditory system. Sound waves cause a displacement of the basilar membrane, and this mechanical vibration is transduced to bio-electrical signals which are transmitted to the brain. Frequency decomposition occurs in the cochlea due to the varying stiffness and width of the basilar membrane. The stiffness of the membrane decreases from the outermost portion of the cochlea to the innermost portion. Sound waves traveling through the cochlea have decreasing velocity due to the stiffness gradient. As the velocity of the sound wave decreases, so does the wavelength. As a result, each position has a resonant frequency, resulting in a position-dependent frequency response along the length of the basilar membrane.

An interesting aspect of the cochlea is the local fluid coupling that occurs [30]. When the basilar membrane undergoes motion in response to an incident sound wave, the motion of the basilar membrane causes a flow in the fluid surrounding it. This fluid flow partially inhibits the response of neighboring portions of the basilar membrane, therefore sharpen- 


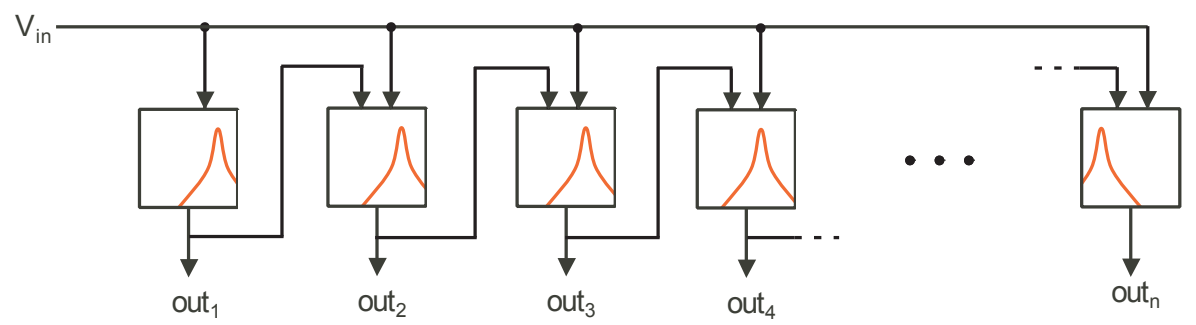

Figure 5.2: Lateral coupling of higher-frequency filters becomes a cascade of filters. Large cascades lead to accumulation problems, such as noise, and should, thus, be avoided. However, this cascade approach should not have as severe problems with accumulation since the lateral coupling has a relatively small gain as compared to the input each filter receives from $V_{i n}$. Also, only a small range of frequencies are passed to the neighboring stages, and, thus, only the noise from those frequency bands are passed to the neighboring stages. Additional lateral coupling can be used from additional stages preceding the filter of interest. As a result, the filter may receive lateral coupling from several of the previous filters.

ing the response of the basilar membrane. In effect, the high-frequency slopes become far greater than would be achieved through a stand-alone resonator, and the overall shape of the tuning curves, or analogously the frequency response, becomes asymmetric about the center frequency with steep high-frequency slopes [31].

\subsection{Silicon Cochlea Models}

Silicon cochleae attempt to model the frequency decomposition that occurs in biological cochleae. Early silicon-cochlea circuits modeled the wave-propagation nature of the basilar membrane via a cascade of lowpass filters [20, 21, 22, 23]. Second-order sections with moderate resonance, or Q, were used for lowpass filters, and the corner frequencies of the filters decreased while moving through the cascade. The transfer functions of the cascaded filters multiply together to yield a very steep transition band. While the cascaded lowpass-filter approach provides the remarkably steep high-frequency slope seen in biology, there is no attenuation of low frequencies, although some models utilized differentiation to achieve a pseudo-bandpass response [21, 22]. In practice, this approach had significant issues such as noise accumulation and the risk of losing functionality of a large portion of the system if one stage stopped working. 
Another approach to modeling the cochlea is simply an array of bandpass filters [32], and several silicon examples are $[24,33,25,34,35]$. Each filter is tuned to a different portion of the audio band and all filters receive the same input. The filters have a narrow bandwidth with moderate- to high-Q. Steeper roll-offs can be achieved by using higher-order filters. While this model does not suffer from cascading issues discussed in the previous section, it does not produce an asymmetrical frequency response as seen in the cochlea.

To model the inhibitory effect of fluid coupling, adjacent filters in the bandpass array can be weighted and subtracted from the filter of concern. Figure 5.1 demonstrates an electronic equivalent to fluid coupling. In this system, a weighted sum of the outputs of each neighboring stage are added to the input of each individual filter. The result is an effective subtraction of the response of the neighboring filters and, thus, a greatly sharpened frequency response from the filter of interest.

While a weighted subtraction on both the low- and high-frequency sides of each particular center frequency would help to sharpen the transitions to the stopband, this lateral coupling is most prominent in the frequencies above the center frequency in biological systems [30]. If only the higher frequencies are to be coupled in, another way to view this system is shown in Fig. 5.2. Each filter in the array receives the common input to the entire system and also the output from the previous stage, which has a slightly higher center frequency. Added input lines could also come from previous stages, as well; however, too many added input lines could cause difficulties in designing the IC for fabrication.

Some of the problems with this approach are illustrated in Fig. 5.2. One major advantage of the array of bandpass filters in parallel was the minimization of accumulation problems that came as a result of a cascade. In the early cascade models [20, 21, 22, 23], significant problems resulted from cascading many lowpass filters. The design of Fig. 5.2 is basically a cascade that also has a common input. This design will also suffer from similar cascade problems, though it will not be as severe since the bandpass filters are passing only the frequencies and noise within their own particular passband. 


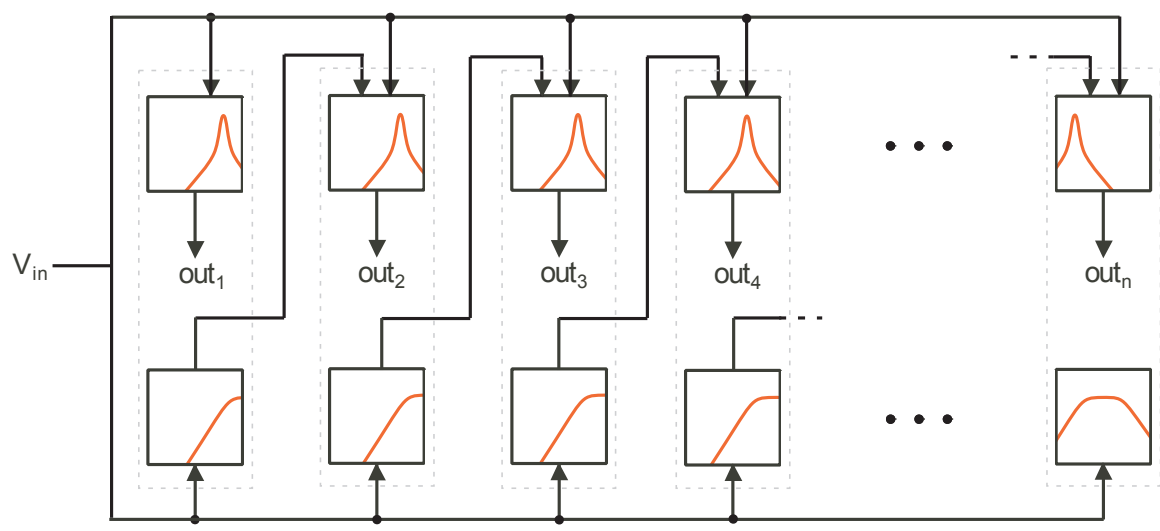

Figure 5.3: Lateral coupling of higher-frequency filters with "dummy" filters. Each filter tap includes an extra filter that is tuned to have a wider bandwidth. This dummy filter receives the same input, $V_{i n}$, as the other filters, but only the dummy filter passes its output to the next filter tap. As a result, this technique has no cascade longer than two filters at any given point. Also, the wide-band filter produces the effect of several cascaded filters since it represents the sum of the preceding filter stages.

\subsection{Lateral Coupling}

The lateral-coupling model presented in this paper achieves the desired response with a minimal amount of cascading. This lateral coupling can be done by adding an extra "dummy" filter along with each filter tap that receives the same input as its associated filter but sends its output to be subtracted from the next stage, as is shown in Fig. 5.3.

This dummy filter is a lower- $Q$ filter than its associated bandpass filter. Also, this filter is set to have a wider bandwidth than its associated filter so that it approximates several of the neighboring filters since the sum of all the higher-frequency filters is essentially the same as a single wide-band filter. The circuit-level schematic of this type of configuration is shown in Fig. 5.4. Specifically, this schematic represents the $\mathrm{C}^{4}$ from the filter tap of interest and the wide-band, lower-Q $\mathrm{C}^{4}$ from the next higher-frequency stage. The buffered output of the dummy filter is capacitively coupled to the main $\mathrm{C}^{4}$. During a tuning, or programming phase, this added capacitor is connected to $V_{d d}$ so that it effectively adds to the capacitance of $C_{W}$; therefore, when the run-time phase is enabled, the effective capacitance seen by each time constant remains fixed, and the corner frequencies do not shift from programming mode to run mode. 
Figure 5.5 shows the simulated and measured results of the lateral-coupling circuit. The narrowband and dummy filters were tuned using the discussion described in the previous section. Once the desired corner frequencies were set, the circuit was connected in lateralcoupling mode. Figure 5.5(a) shows the measurement and simulation data for each component of one filter tap, including the narrowband filter with no lateral coupling, the dummy filter, and the filter with lateral coupling. As can be seen, the laterally coupled $\mathrm{C}^{4}$ has a much faster high-frequency roll-off than does the nominal $\mathrm{C}^{4}(-40 \mathrm{~dB} /$ decade as opposed to $-20 \mathrm{~dB} /$ decade). Also, the output of the dummy filter is clearly shown to be a wide bandwidth response, which simulates the effect of laterally coupling several of the higher-frequency stages.

Once the required relationship between the corner frequencies of the two $\mathrm{C}^{4}$ 's was determined, the bias voltages were swept to emulate an array of laterally coupled filter taps (Fig. 5.5(c)). An exponential spacing of center frequencies (seen in the cochlea) was achieved by linearly sweeping the bias voltages to get an exponential sweep of bias currents (subthreshold). An array of these filters could thus be fabricated to provide the same overall response.

Our final measurement cascaded the lateral coupling filter with a third $\mathrm{C}^{4}$ tuned to the same frequency response of the narrowband filter. An attenuating circuit was used to keep the signal in the linear range of the cascaded $\mathrm{C}^{4}$. Cascading the $\mathrm{C}^{4}$ 's increased the order of the filter to yield a 40dB/decade slope going up and a -60dB/decade slope going down, as seen in Fig. 5.5(b). 


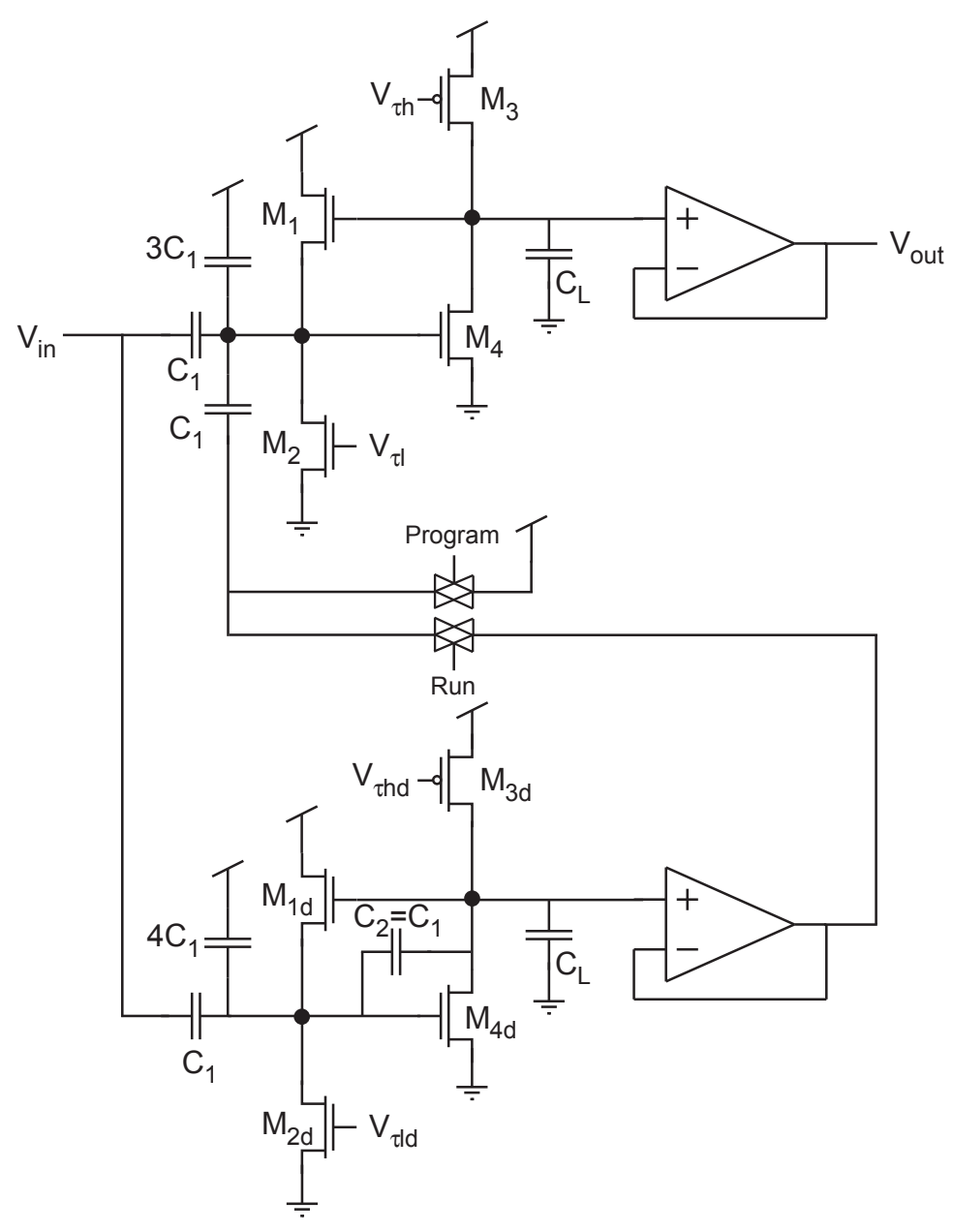

Figure 5.4: Schematic of the effective circuit for each lateral-coupling filter tap. This schematic shows the $\mathrm{C}^{4}$ of the representative filter tap and the dummy filter from the next higher-frequency stage. The main $\mathrm{C}^{4}$ receives a capacitively coupled input from the dummy filter. The second input capacitor is connected to $V_{d d}$ during programming, or tuning, mode so that it effectively adds to the total value of $C_{W}$, and the corner frequency will not change from tuning mode to run mode. In order to increase the $\mathrm{Q}$ and gain of the top $\mathrm{C}^{4}$, the $C_{2}$ capacitor was removed. 


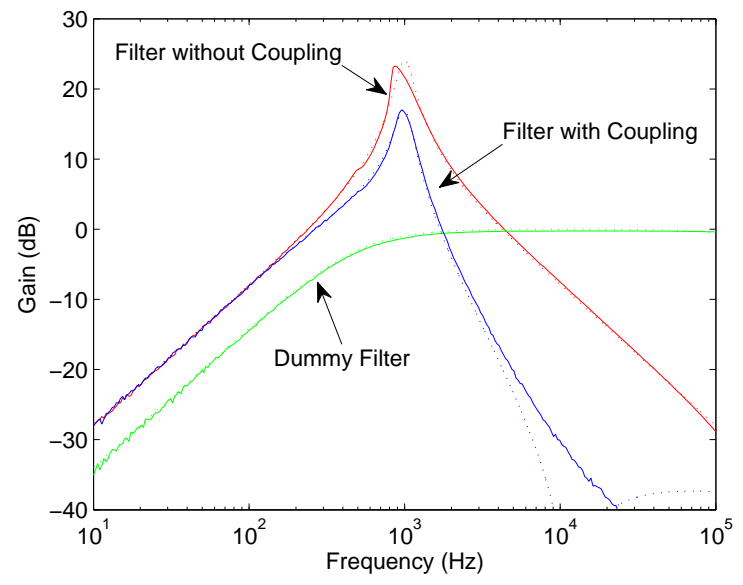

(a)

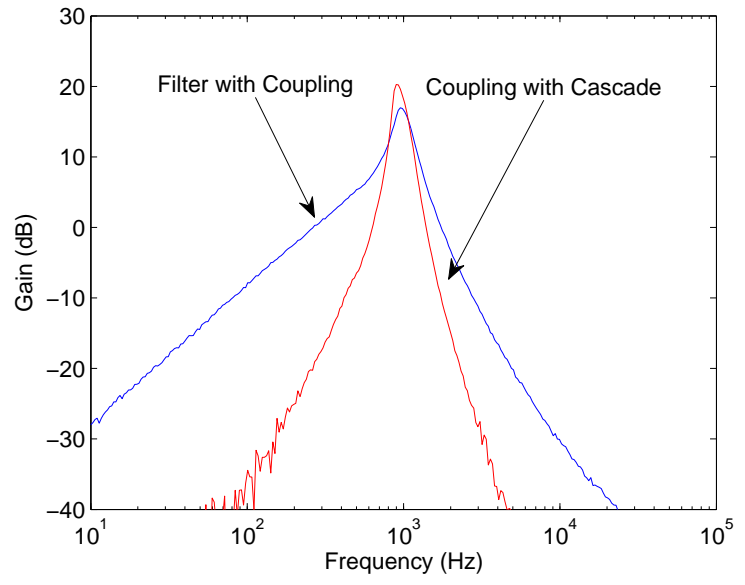

(b)

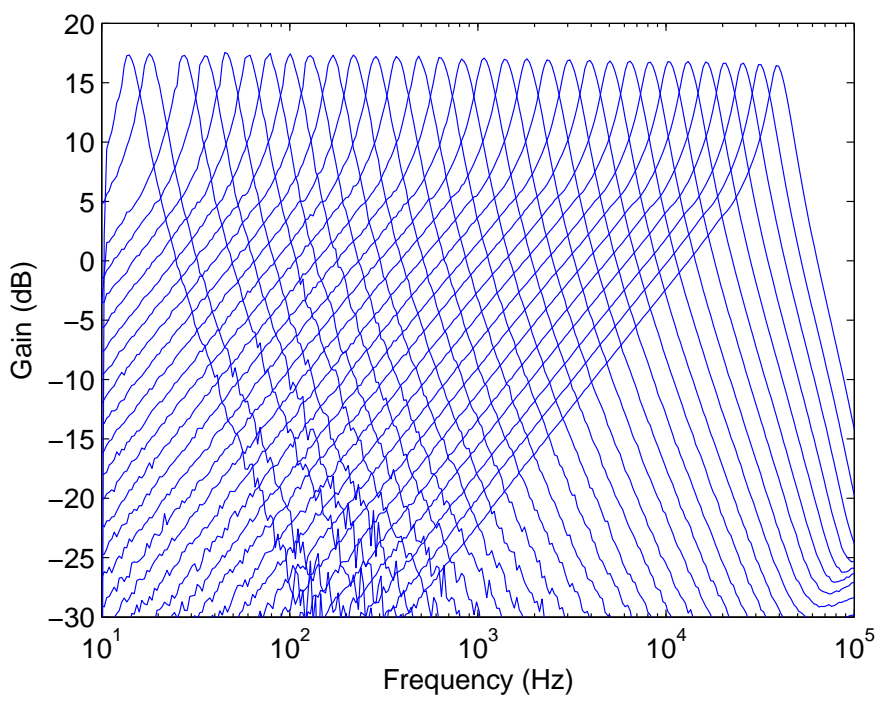

(c)

Figure 5.5: (a) Individual frequency response for each filter in the lateral-coupling circuit as well as the frequency response for the entire filter tap. The center frequency is set to $1 \mathrm{kHz}$. Simulation results are represented by dashed lines and actual data is represented by solid lines. (b) Frequency sweep showing the relationship between a second-order lateralcoupling stage and a fourth-order lateral-coupling stage. The signal was attenuated after the output of the lateral-coupling circuit to remain in the linear range of the cascaded $\mathrm{C}^{4}$. The slope of the fourth-order lateral-coupling stage is $20 \mathrm{~dB} /$ decade steeper on each side, resulting in $40 \mathrm{~dB} /$ decade going up and $-60 \mathrm{~dB} /$ decade going down. (c) Frequency response measurements of the lateral coupling circuit. One filter tap was used and the bias voltages were swept linearly to change the center frequency for each frequency response curve. 


\section{Chapter 6}

\section{Low-power Hardware-based Event Detection}

A great application of analog front-ends is in the context of wireless sensor networks. Sensor nodes (motes) typically have limited computational resources and very restrictive power requirements. They are often deployed in environments where battery changes are not possible. Future motes are planned to run entirely from energy scavenged from the environment [36]. Low-power analog processing can extend the functionality of sensor nodes without increasing the energy needs of the mote.

In sensor networks the nodes maintain a low wake/sleep duty-cycle to reduce power consumption. A mote wakes up for short durations to sample sensors and communicate with other nodes. The problem with this approach is that a short event of interest may be missed because all of the nodes are asleep. A solution for this is to perform event detection in alwayson, low-power hardware which can wake up the mote when an event occurs. Detection may be as simple as waking the mote anytime a sensor's signal exceeds some threshold [37] (i.e. any sound), or may involve classifying events (i.e. distinguishing between a footstep and a voice) for more discriminating event detection. 


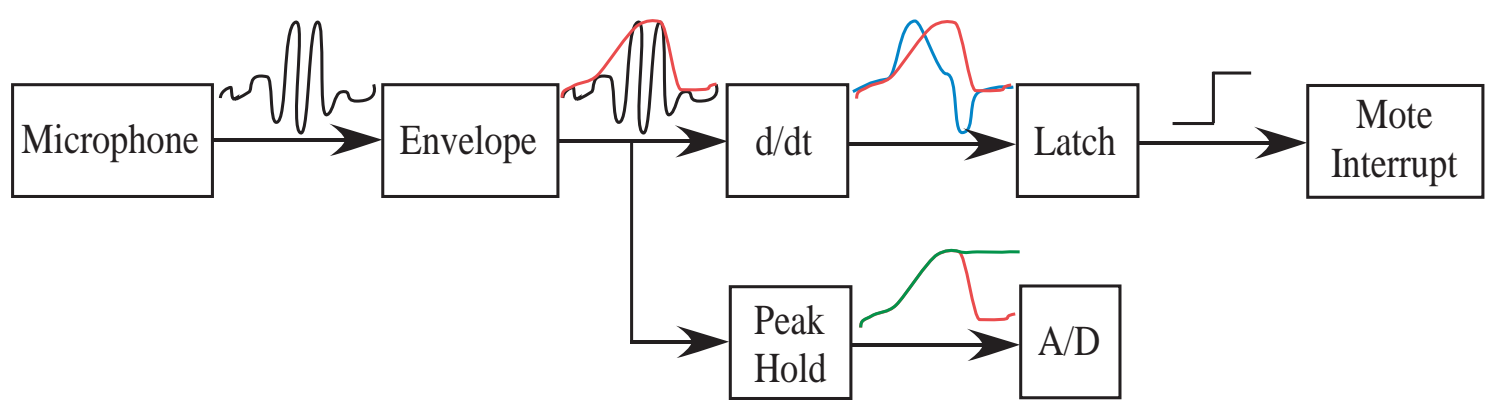

Figure 6.1: Idea for hardware detection of sound events.

\subsection{Sound Threshold Event Detection}

A simple sound event detector has been prototyped with discrete components. The initial idea for sound onset detection (fig. 6.1), was to take the derivative of the magnitude to find large changes in the energy of the signal, which would correspond to a sound onset. When an onset is detected, a hardware interrupt is signaled to the microcontroller on the mote. The mote can sleep through all of this and when the interrupt occurs it wakes up to process the event. In the lower path of the system (fig. 6.1), the output of the magnitude detector goes into a sample and hold, so that the magnitude of the signal at the time of an event can be saved for when the mote wakes up. Unfortunately, this project was done before the magnitude circuit in chapter 3 was back from the foundry, so the peak detector was constructed from discrete components. This breadboarded peak detector did not include the nonlinear filter, and its output had a sawtooth shape which caused bogus results when fed to a differentiator.

The actual working bread-boarded detection system consisted of a microphone, amplifier, magnitude detector, and a comparator. When the magnitude of the signal exceeds the reference voltage, the comparator output goes high. The power consumption of the entire circuit (microphone, microphone amplifier, magnitude, and comparator) was around $3 m W$. The circuit without the microphone could be condensed into an integrated circuit to reduce the power consumption to the order of $\mu W^{\prime}$ 's, however the limiting factor seemed to be the microphone which consumed over $1 \mathrm{~mW}$.

Two circuits were built and each connected to an IRIS mote via an MDA300 sensor 


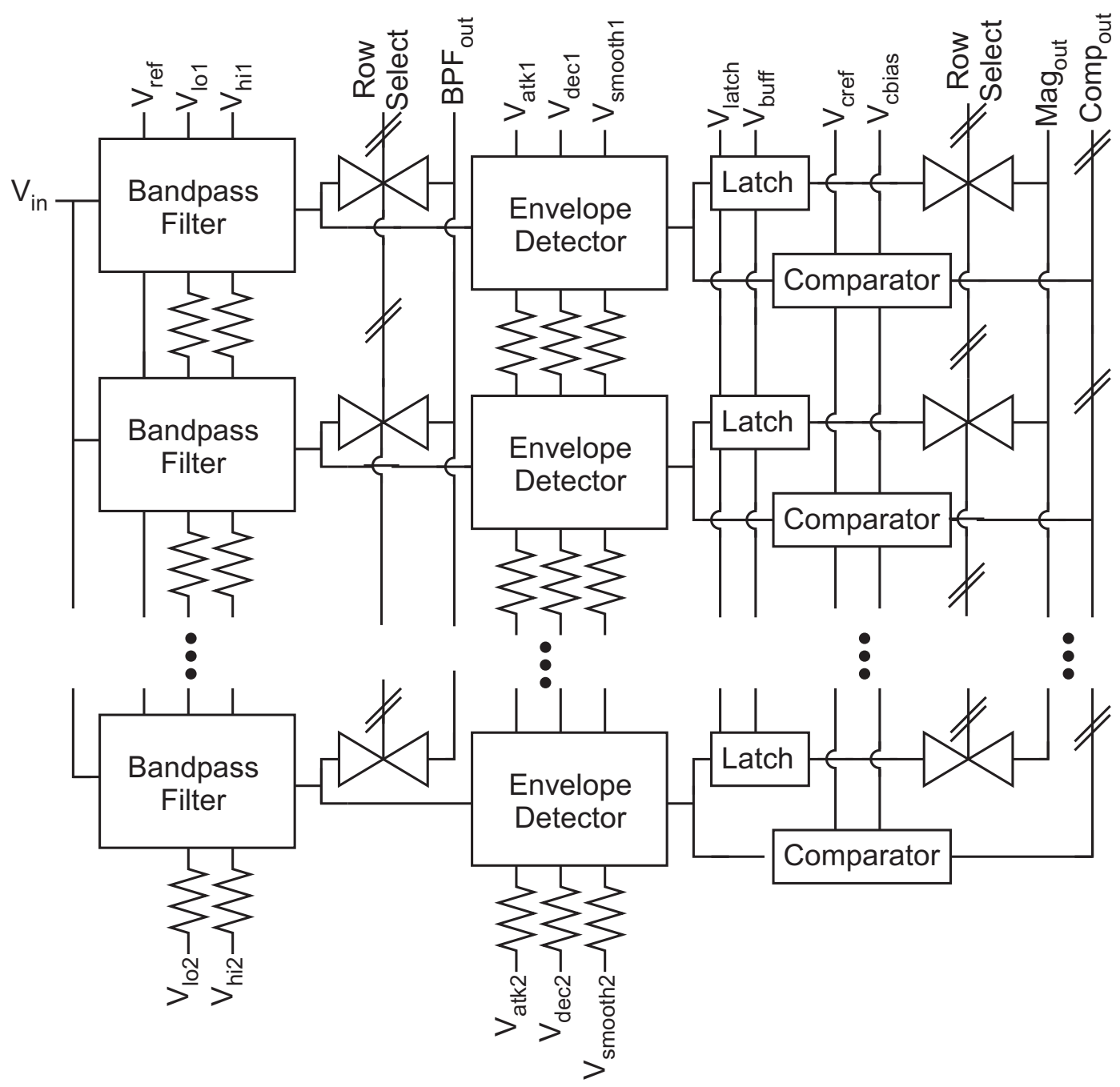

Figure 6.2: Block diagram for the fabricated event detection system.

board. The comparator output was used to trigger a TinyOS event upon receiving an interrupt. When the TinyOS event is triggered, the mote transmits the time of the event to a base-station. In addition, the base-station sends a packet every 10 seconds to synchronize the motes timers. The base station was connected to a PC running Matlab. A Matlab routine was written to determine the location of the sound (in one-dimension) using the time it was detected at each node. 
Brandon D. Rumberg

\subsection{Sound Event Classification}

A sound event detection/classification front-end (fig. 6.2) has been fabricated in a $0.5 \mu m$ process (fig. 6.3). The system has an eight channel filter bank which was designed for a maximum gain of $18 d B$ to avoid the need for the microphone amplifier. However, the filter bank can be biased for any gain from $0 d B$ to $18 d B$ while maintaining a $Q>3$. Demonstrations of this filter bank are shown in chapter 4. For simplicity the biases are set via an on-chip resistive-divider. Each sub-band consists of the magnitude detector presented in chapter 3, a comparator, and a latch to sample the spectrum at the time of the event, and hold the spectral characteristics of the event until the mote wakes up to read each sub-band. The comparator reference can be adjusted independently for each pair of channels.

This system was built as an initial demonstration of the capabilities of hardware-based event detection and classification. Since each comparator output goes to a separate pin, their output can be fed into a logic circuit to generate a mote wakeup pulse when desired spectral characteristics are detected. An initial (and contrived) demonstration will be to use tones centered in different frequency bands. Let's say two tones, tone A and tone B, for which there are three classes of events, just tone A, just tone B, and both tones combined. We might define the classes of interest as being just tone $\mathrm{A}$ or tone $\mathrm{B}$, and perform an exclusive-or on the comparator outputs of those two sub-bands to distinguish between the possible event classes.

\subsection{Potential directions}

The event classification system discussed in the previous section has many possibilities, but also has several shortcomings. The first update is to replace the resistive biasing with floating-gate biasing. Floating-gate biases will allow the filter bank to be programmed for configurations other than constant-Q and constant-gain. For example, one channel could be tuned to the full-band, or channels could be tuned to isolate certain frequencies of interest. Another problem with resistive biasing is the lack of precision. Since the DC level of the magnitude's output is dependent on it's biasing, the imprecise resistive divider results in 
each channel having a different DC level, making it difficult to compare the sub-bands.

Several analog classification building blocks have been reported in the literature, such as vector quantization [38, 39] and hidden Markov models [10]. Replacing the comparators in our system with soft-logic should increase the possibilities for classification. Also because of the significance of timing in sounds, classification performance would be enhanced by using classification schemes which have memory. 


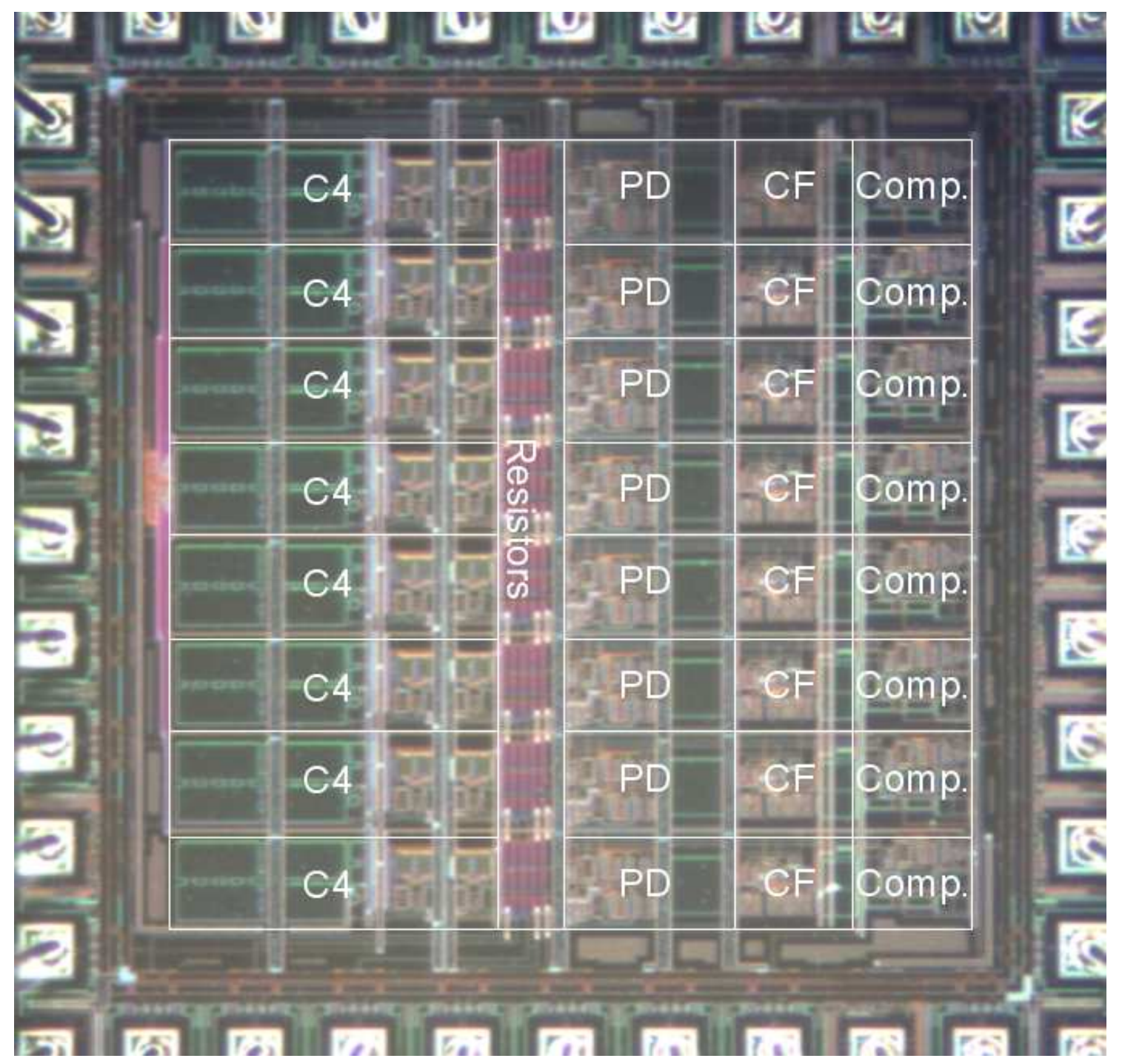

Figure 6.3: Die shot of the event detection chip. The area inside the pad frame measures $1.5 \mathrm{~mm} \times 1.5 \mathrm{~mm}$ 


\section{Chapter 7}

\section{Conclusion / Future Directions}

In this work I have presented the design and results of a low-power analog integrated circuit which performs frequency decomposition for audio processing. This spectral decomposition system required the development of a bandpass filter and a magnitude detector. The bandpass filter is low-power, easy to use, and can be programmed for different quality factors and passband gains. Efficient, constant-Q filter banks have been built by arraying these filters. The magnitude detector operates over a large dynamic range and is able to follow power changes in realistic speech signals very accurately. By including the magnitude detector in the filter bank's sub-bands, I have constructed full frequency decomposition front-ends.

We have begun experimenting with the applications of these circuits. Plans for the future are to explore the application of these front-ends for hardware-based event detection and noise suppression. We hope that integrating these front-ends into complete systems will yield new insights, and guide future iterations. At present, we are working to include features such as gain and $\mathrm{Q}$ adaptation to increase the dynamic range of the front-ends. We will also be exploring alternatives to second-order filters, such as higher-order, gammatone, or wavelet filters. In the future, we will include post filter bank circuitry on-chip to perform feature extraction such as the mel-frequency cepstrum. We will also work to include analog classification elements to perform auditory scene analysis. It is hoped that this work will be beneficial for hearing prostheses, mobile communications devices, sensor networks, as well as unforeseen applications. 


\section{Appendix A}

\section{Alternate Magnitude Circuits}

In the process of developing the magnitude detector presented in chapter 3, several alternative circuits were developed which were deemed inappropriate for our application. Three of those circuits are briefly discussed in this section. All plots in this section are from circuits fabricated in a $0.5 \mu m$ process.

\section{A.1 Asymmetric-OTA Peak Detector}

In chapter 3 we showed how a peak detector with amplitude dependent decay could be made. The current onto the capacitor for that peak detector was effectively an asymmetric tanh. One way to achieve an asymmetric tanh is by ratioing the input pair and current mirrors in an OTA (fig. A.1(a)). The ratio between the attack and decay is set by $N$, which is the strength ratio for the input pair and the pFET current mirror. This circuit was fabricated in a $0.5 \mu \mathrm{m}$ process with $N=15$, the I-V curve is shown in fig. A.1. Figure A.2(a) shows the asymmetric-OTA peak detector's response to an amplitude modulated sine wave, the "magnitude" trace is the combined response of this peak detector and the nonlinear filter. Note that this peak detector still maintains an amplitude dependent decay, which is further demonstrated in fig. A.2(b).

If it is desired to be able to change the attack to decay ratio, we can use floating-gate transistors for the input pair and the pFET current mirrors. With floating gates, $N$ can be programmed precisely. A floating gate version was also fabricated in a $0.5 \mu \mathrm{m}$ process 


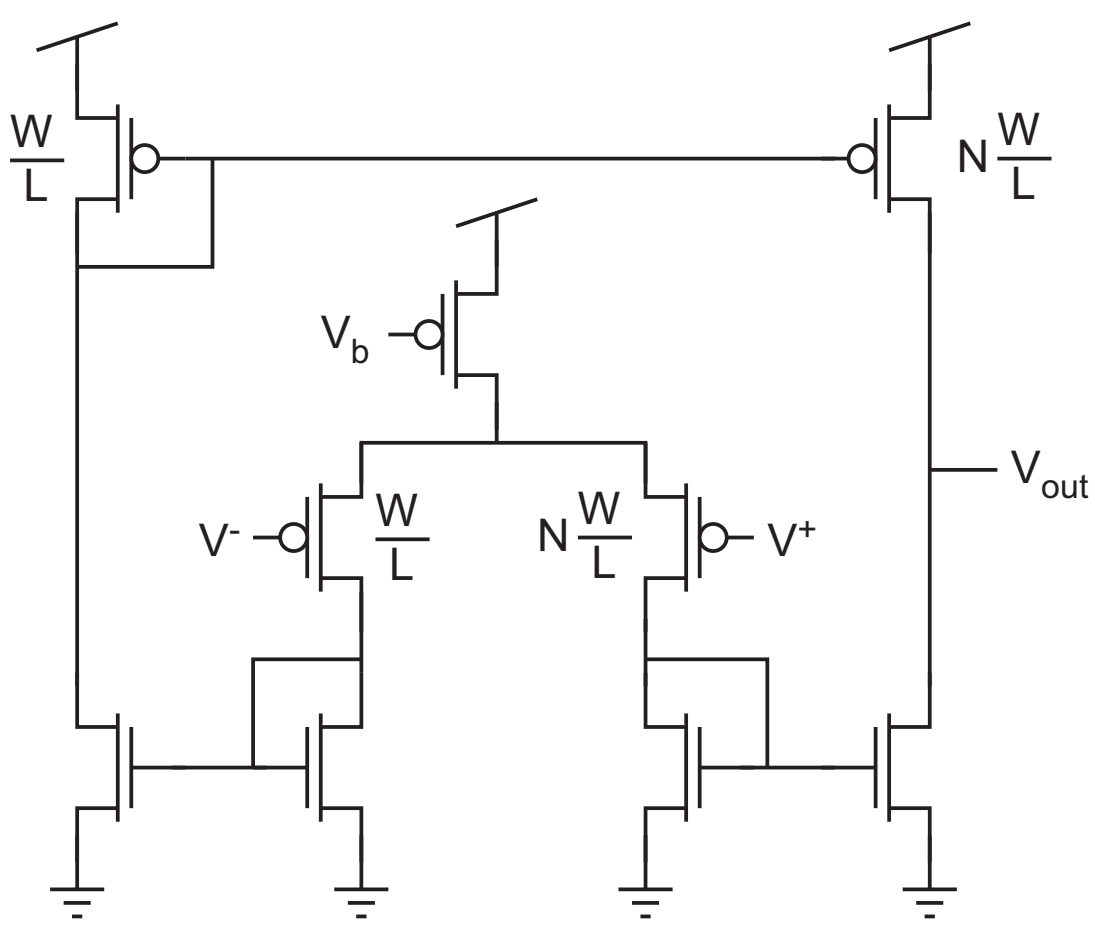

(a)

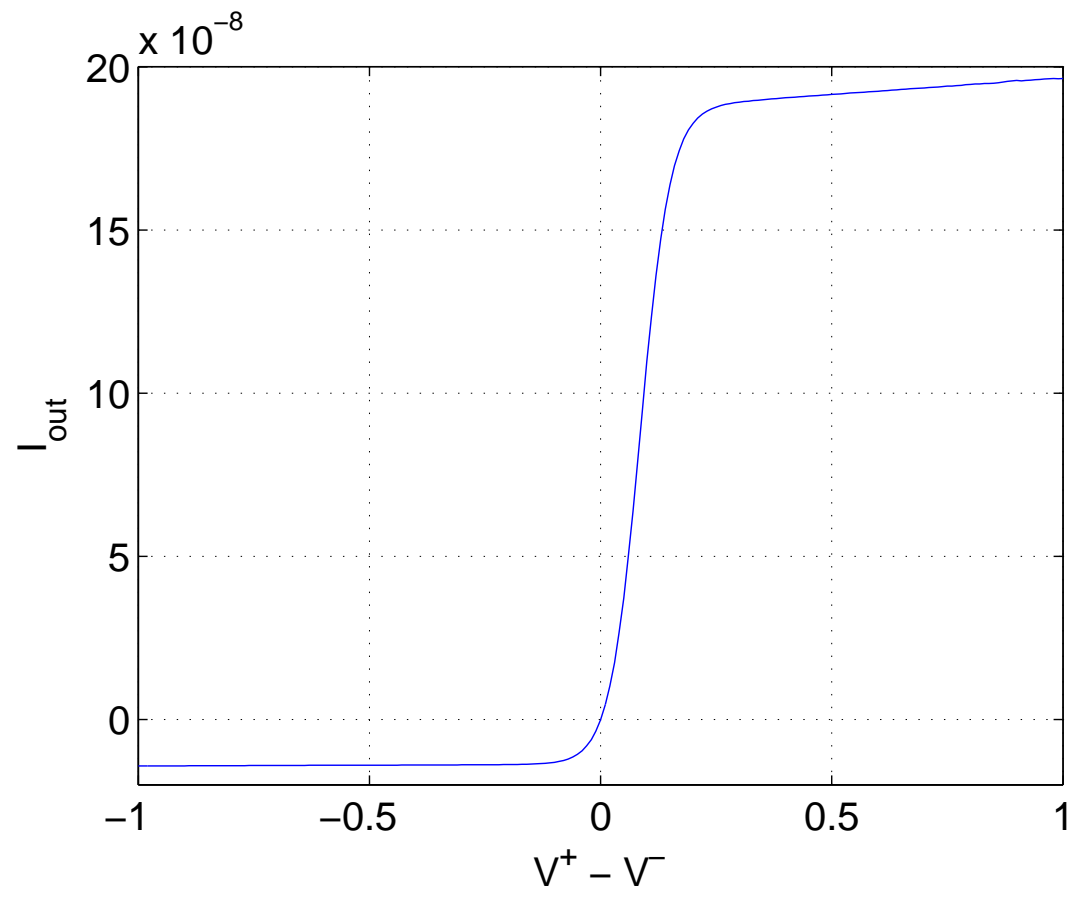

(b)

Figure A.1: (a) Schematic of the asymmetric OTA (b) I-V curve of the asymmetric OTA. 


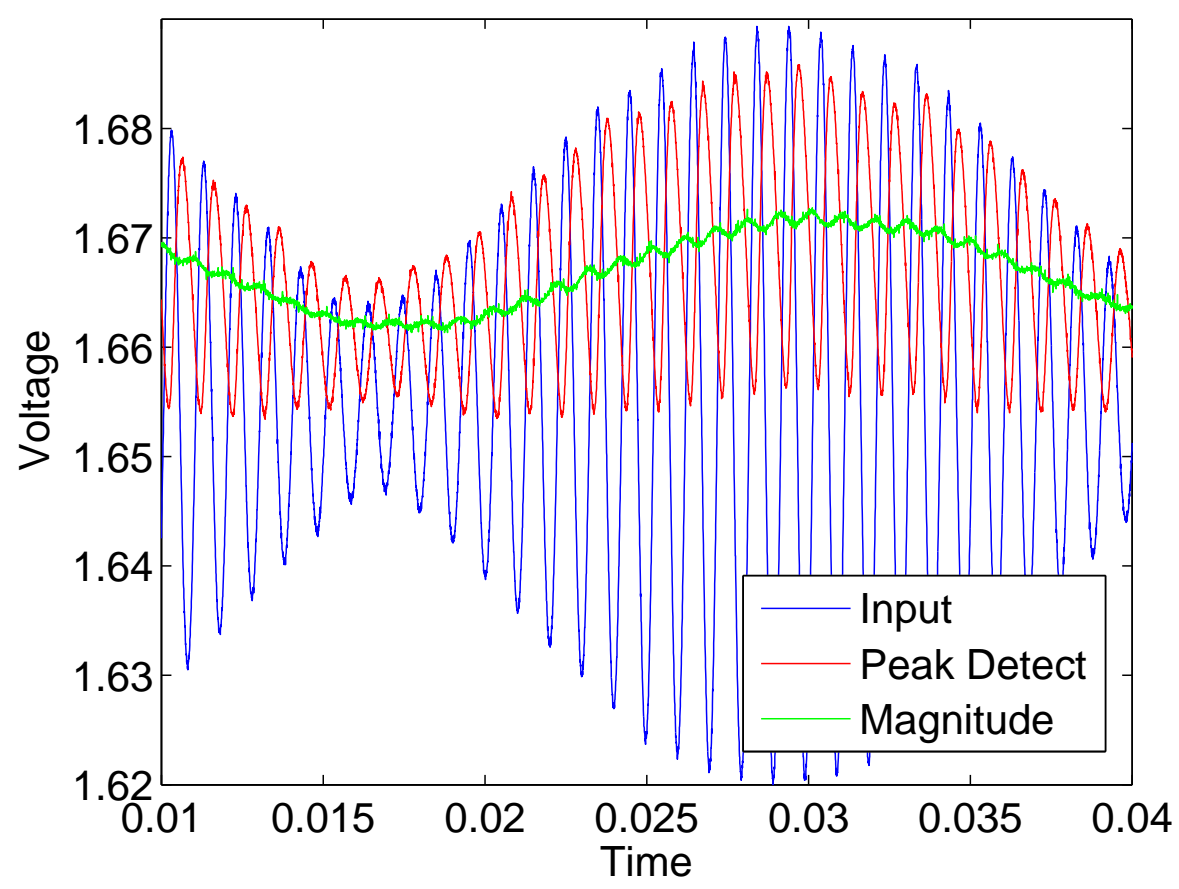

(a)

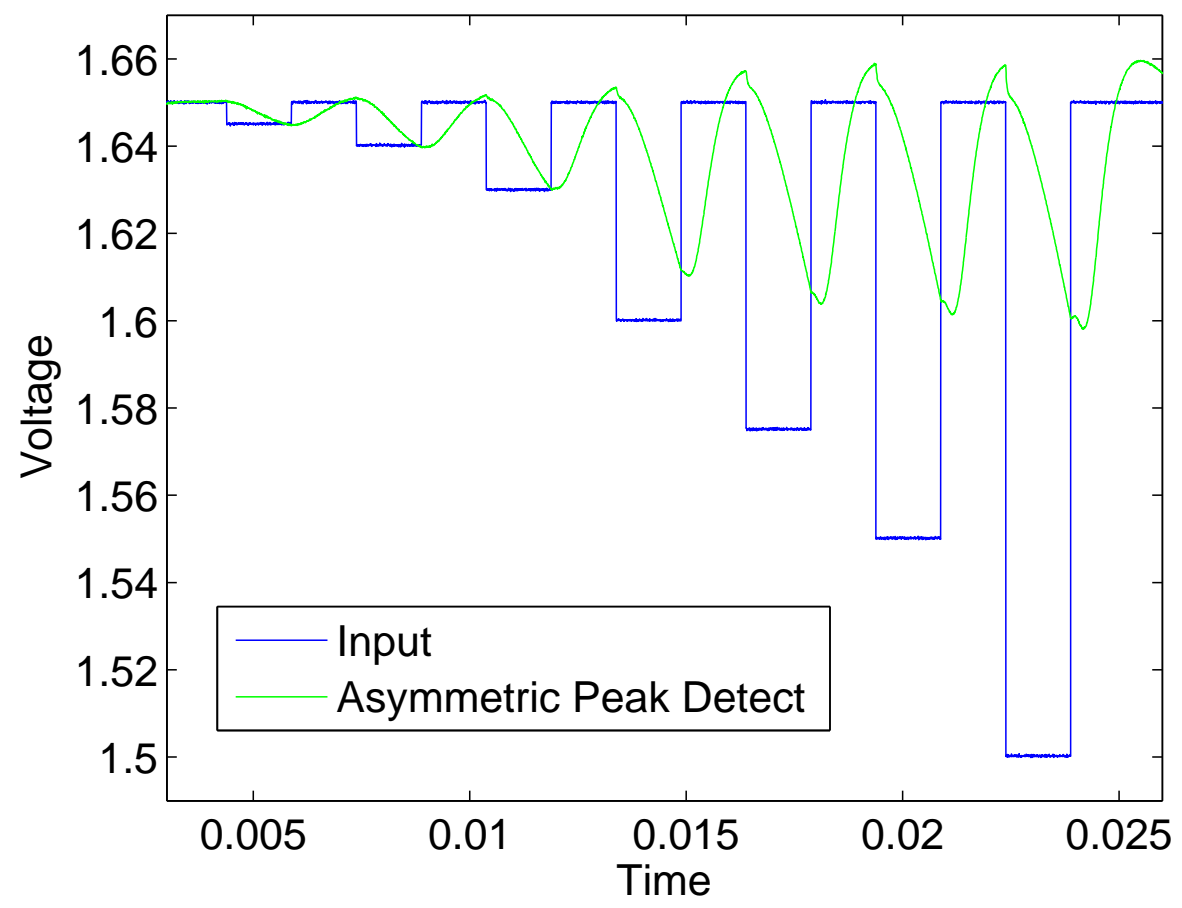

(b)

Figure A.2: Response of asymmetric-OTA peak detector to (a) an amplitude modulated sine wave, and (b) downward steps. 


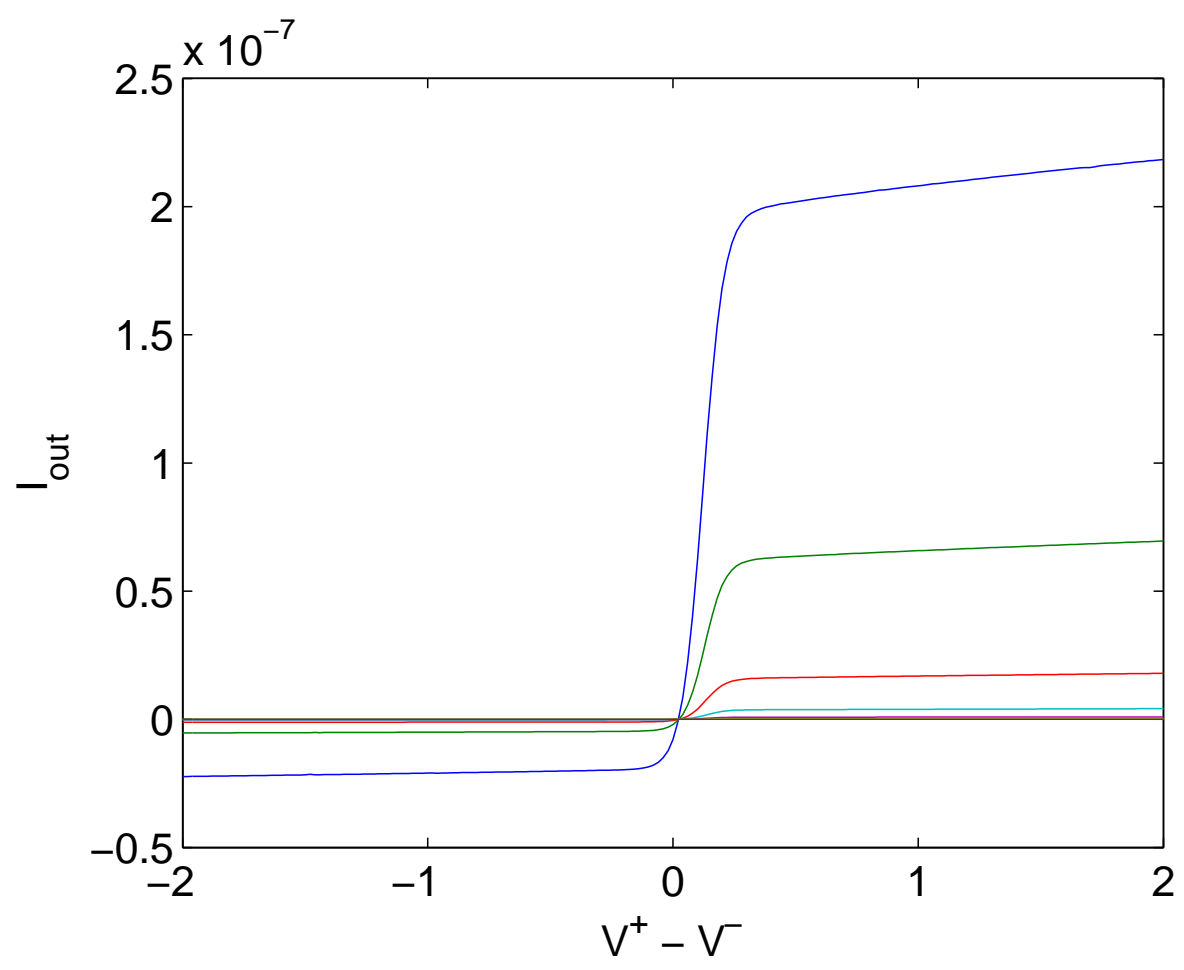

(a)

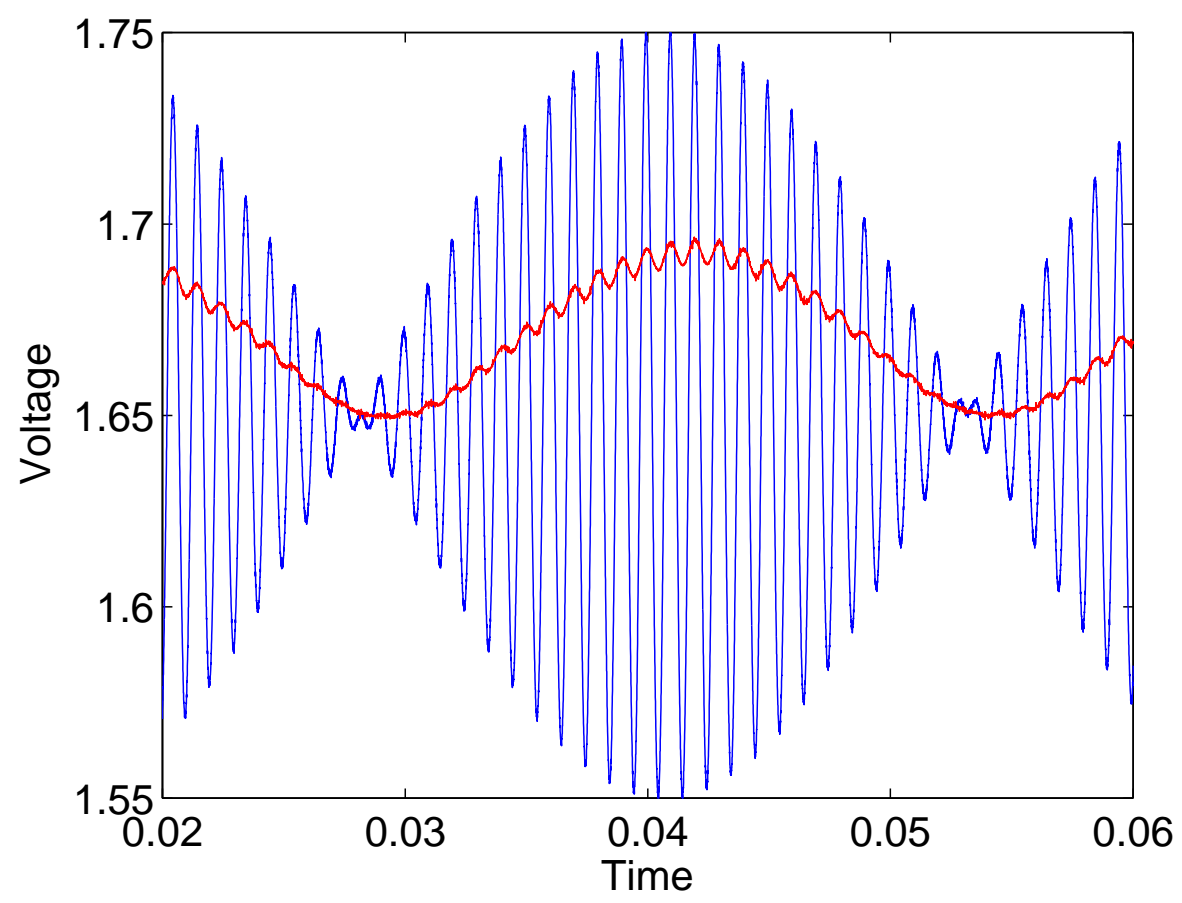

(b)

Figure A.3: (a) I-V curves for a floating-gate based asymmetric OTA. (b) Response of floating-gate asymmetric-OTA peak detector to an amplitude modulated sine wave. 
and was programmed to $N=20$ for these demonstrations. The I-V curve for the floatinggate asymmetric OTA is shown in fig. A.3(a). Figure A.3(b) shows the response of the floating-gate asymmetric-OTA peak detector to an amplitude modulated sine wave.

\section{A.2 Full-Wave Peak Detector}

Our magnitude circuit (chapter 3) performed half-wave rectification, but it is simple to extend to full-wave rectification as shown in fig. A.4(a). Two "constant decay" peak detectors are used to detect positive and negative peaks. The negative peak detected current is then added to the positive peak detector. The operation of this circuit is demonstrated in fig. A.4(b). An amplitude modulated sine wave is used for the input. The "Negative Peak Detect" trace is the negative peak detected signal at node $V_{\text {out,bottom }}$, and the "Positive+Negative Peak Detect" trace is the full-wave peak detected signal at node $V_{\text {out,top }}$. The "Magnitude" trace is the response of the nonlinear filter. This full-wave peak detector yields an increase in performance, but at the expense of doubling power and size.

\section{A.3 Peak Hold Detector}

Figure A.5 shows a circuit which can detect and hold peaks. This circuit performs peak detection with the "adaptive decay" peak detector from chapter $3 . G_{m 3}$ compares the input with the peak detected output. The inverter, NOR gate, and $C_{\text {delay }}$ perform falling edge detection and output a short pulse when the peak detector transitions from attacking to decaying (i.e., the peak). The pulse (which is coincident with the peak) is used to sample the peak voltage onto $C_{\text {out }}$.

The peak-hold circuit's operation is demonstrated in fig. A.6(a). The input is an amplitude modulated sine wave. The peak detector operates as normal. The peak-hold circuit detects each peak asynchronously, and holds the peak value until the next peak. Figure A.6(b) shows the operation of the peak-hold detector combined with the nonlinear filter. The input is an amplitude modulated pulse train. The peak-hold samples each peak and the nonlinear filter smooths it out to recover the modulating waveform. 


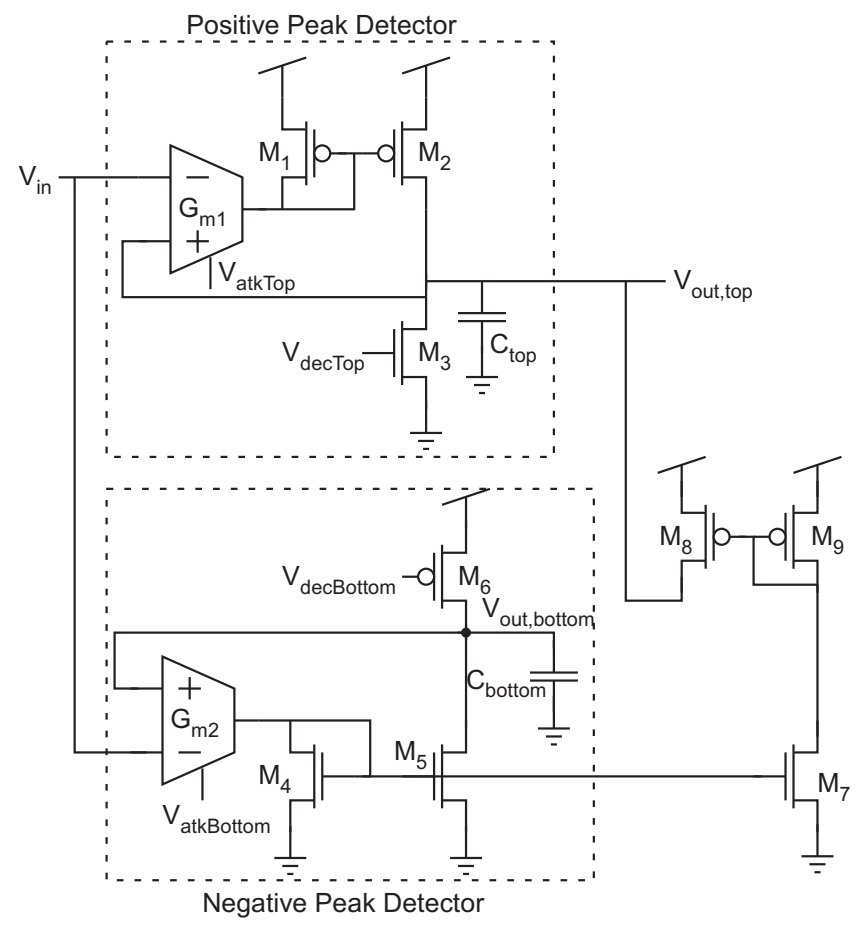

(a)

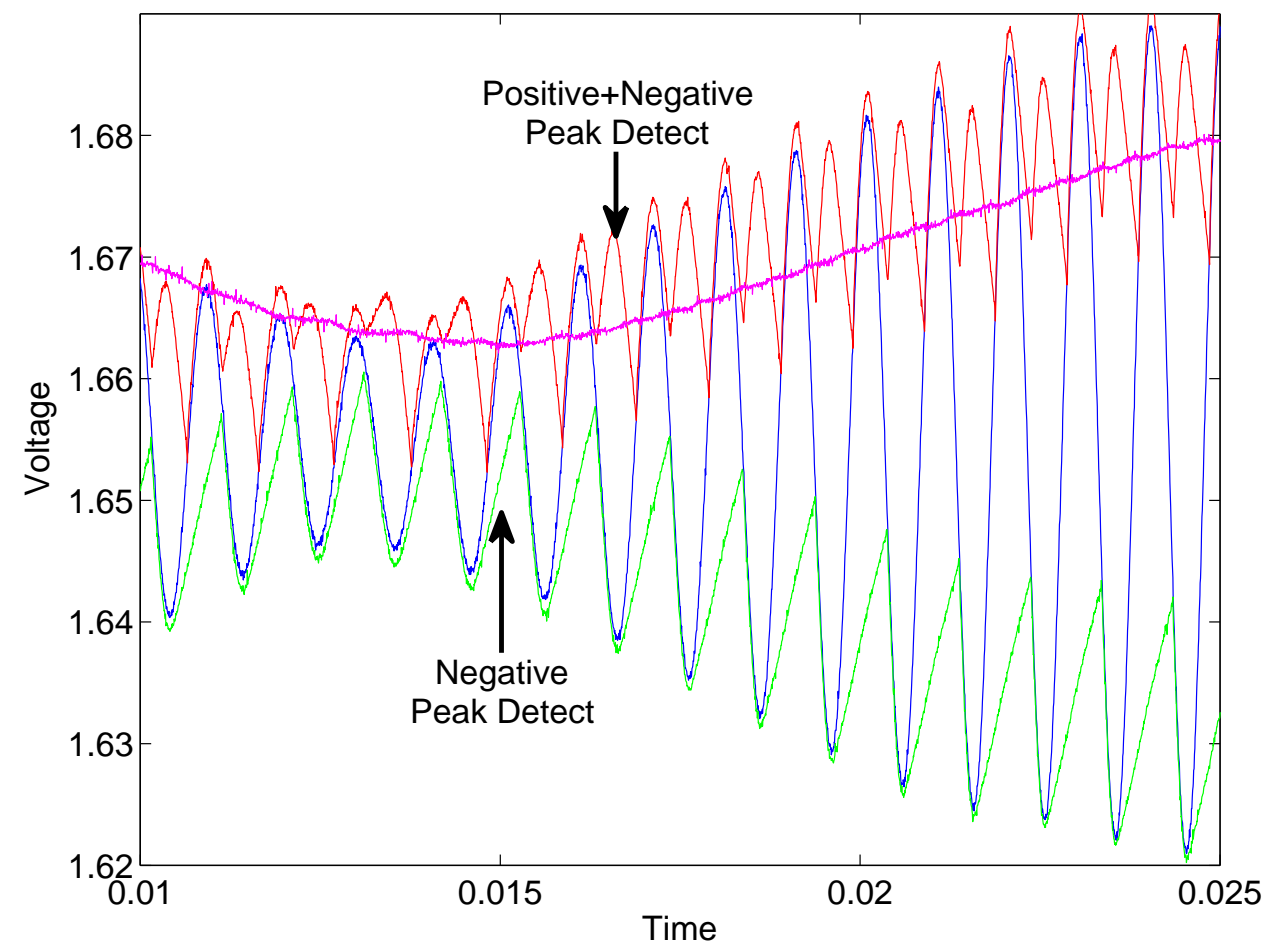

(b)

Figure A.4: (a) Schematic of the full-wave peak detector. (b) Response of the full-wave peak detector to an amplitude modulated sine wave. 


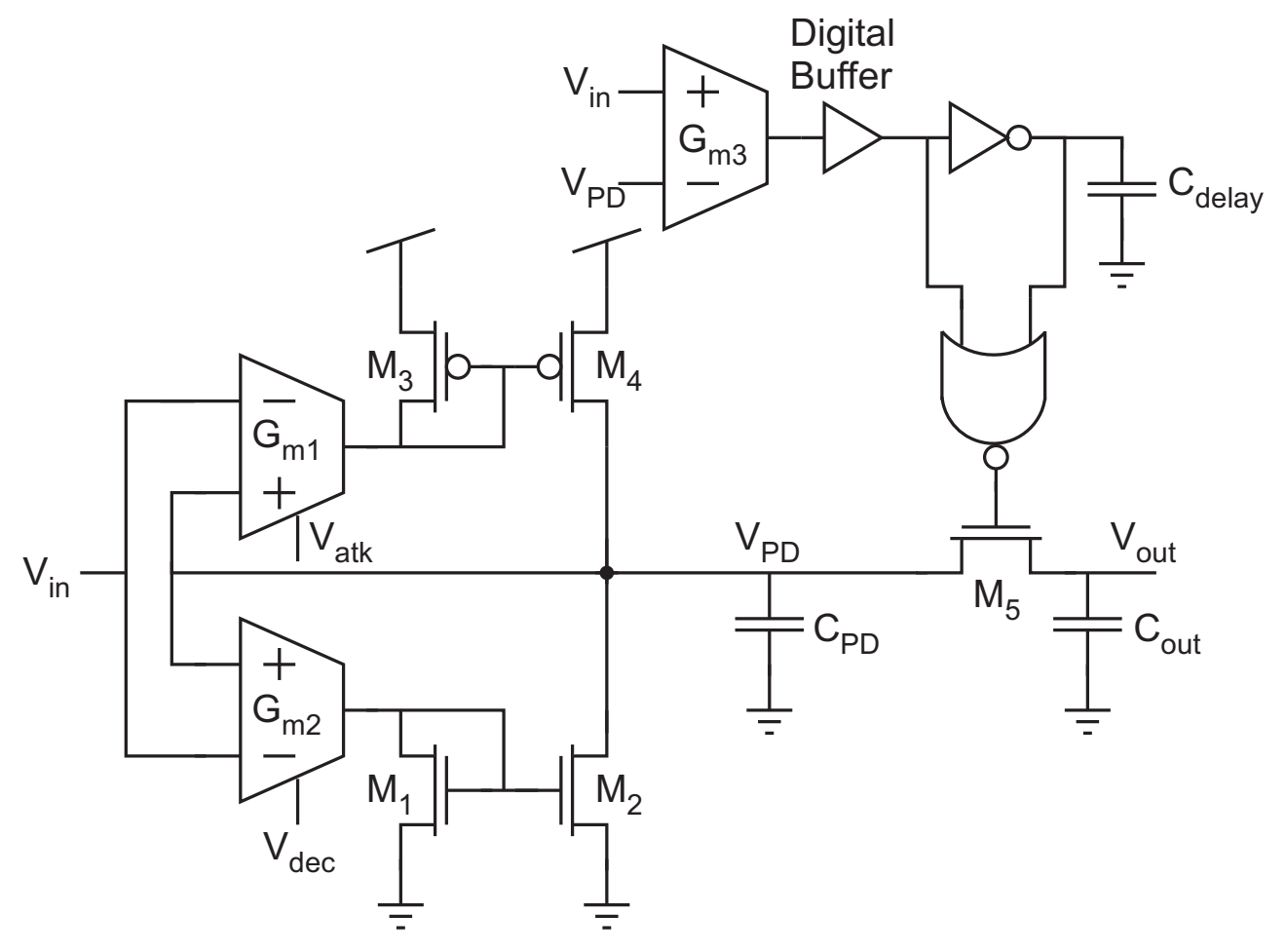

Figure A.5: Peak-hold detector 


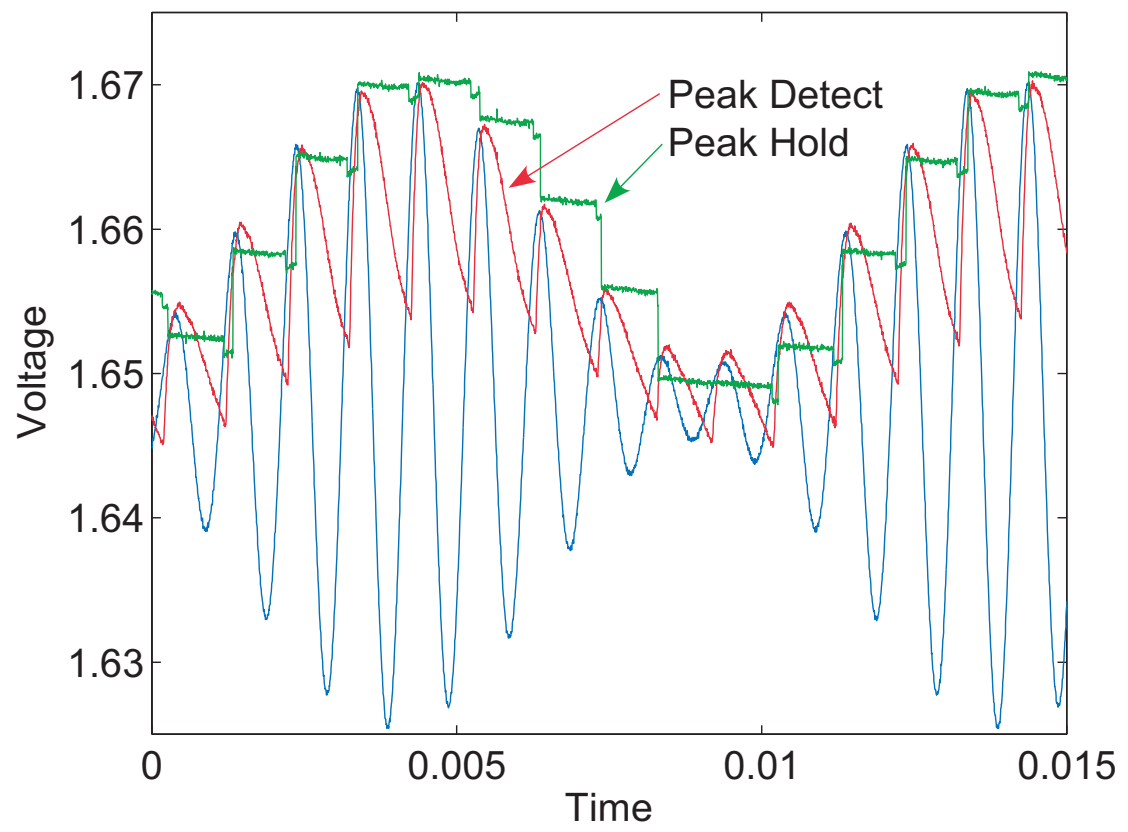

(a)

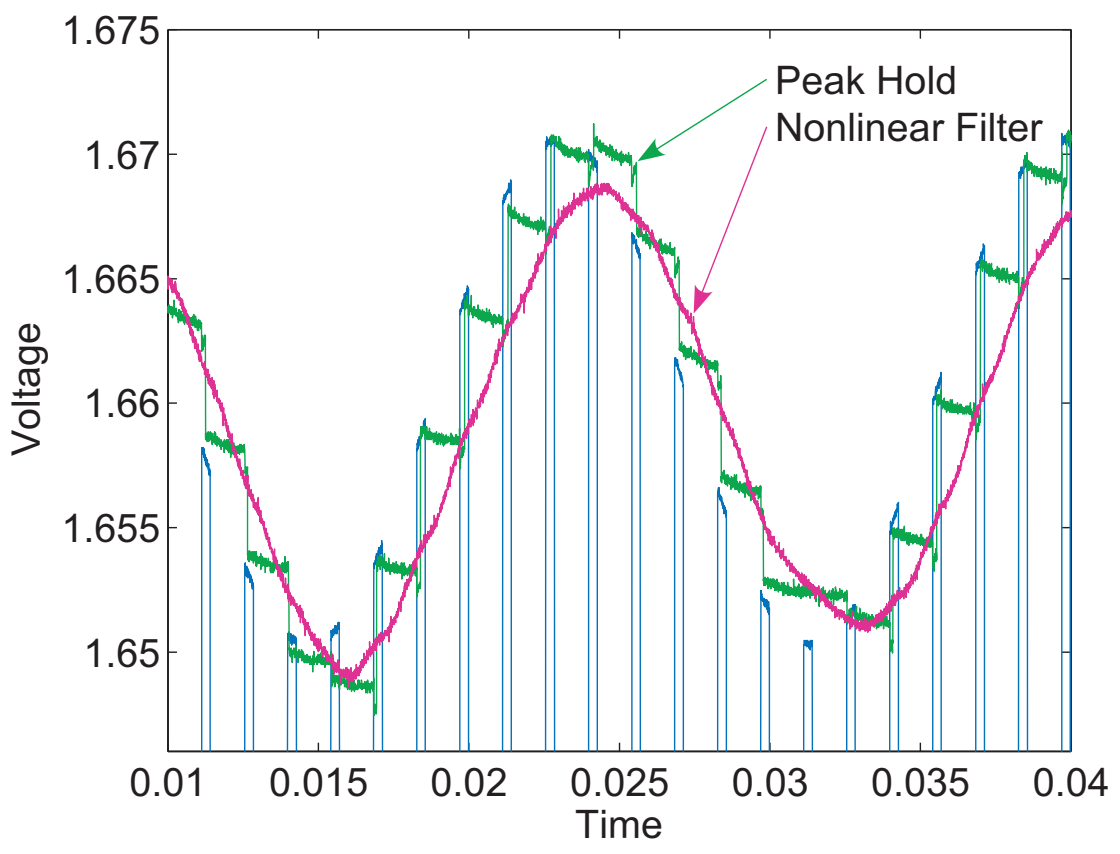

(b)

Figure A.6: Peak-hold detector response to an amplitude modulated (a) sine wave, and (b) pulse train. 


\section{Appendix B}

\section{$C^{4}$ Derivations}

\section{B.1 Derivations for an OTA based $\mathrm{C}^{4}$}

What follows is a derivation of the equations describing an OTA-based $\mathrm{C}^{4}$. In an OTAbased $\mathrm{C}^{4}$, OTA's replace the source follower and common-source amplifier in the normal $\mathrm{C}^{4}$. In the following sections we will derive the transfer function, time constants, $Q_{\max }$, and the relationship between $Q_{\max }$ and the transconductances and capacitances.

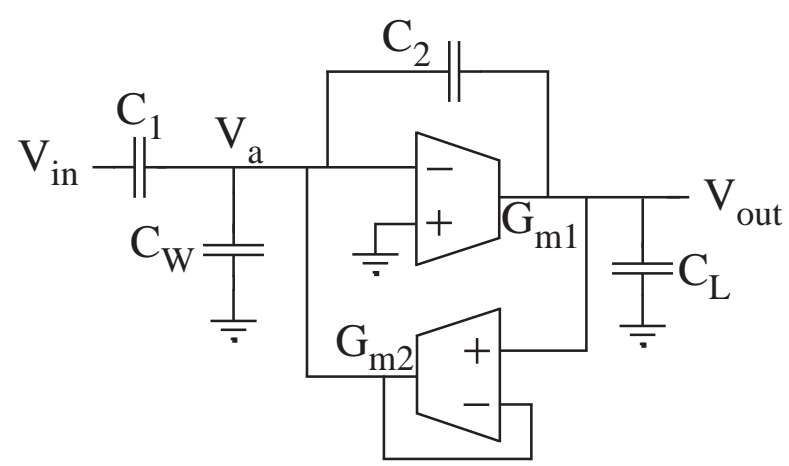

Figure B.1: Schematic of the OTA-C ${ }^{4}$ for transfer-function derivation. 
Brandon D. Rumberg

Appendix

75

\section{B.1.1 Transfer Function}

First we will apply KCL at nodes $V_{a}$ and $V_{\text {out }}$. At node $V_{a}$ we have

$$
\begin{aligned}
s C_{1}\left(V_{a}-V_{\text {in }}\right)+s C_{W} V_{a}+s C_{2}\left(V_{a}-V_{\text {out }}\right) & =G_{m 2}\left(V_{\text {out }}-V_{a}\right) \\
V_{a}\left(s\left(C_{1}+C_{2}+C_{W}\right)+G_{m 2}\right)-s C_{1} V_{\text {in }} & =V_{\text {out }}\left(G_{m 2}+s C_{2}\right)
\end{aligned}
$$

and at node $V_{\text {out }}$ we have

$$
\begin{aligned}
s C_{L} V_{\text {out }}+s C_{2}\left(V_{\text {out }}-V_{a}\right) & =-G_{m 1} V_{a} \\
V_{\text {out }}\left(s C_{L}+s C_{2}\right) & =V_{a}\left(s C_{2}-G_{m 1}\right)
\end{aligned}
$$

Combining equations B.2 and B.4 we get

$$
V_{\text {out }}\left(\frac{s\left(C_{L}+C_{2}\right)\left(s\left(C_{1}+C_{2}+C_{W}\right)+G_{m 2}\right)}{s C_{2}-G_{m 1}}-G_{m 2}+s C_{2}\right)=s C_{1} V_{\text {in }}
$$

We define $C_{T}$ and $C_{O}$ as

$$
\begin{aligned}
& C_{T}=C_{1}+C_{2}+C_{W} \\
& C_{O}=C_{2}+C_{L}
\end{aligned}
$$

Simplifying equation B.5 yields

$$
\frac{V_{\text {out }}}{V_{\text {in }}}=\frac{s \frac{C_{1}}{G_{m 2}}\left(s \frac{C_{2}}{G_{m 1}}-1\right)}{s^{2} \frac{C_{O} C_{T}-C_{2}{ }^{2}}{G_{m 1} G_{m 2}}+s\left(\frac{C_{L}}{G_{m 1}}+\frac{C_{2}}{G_{m 2}}\right)+1}
$$

The transfer function of a generic bandpass SOS is

$$
H(s)=A_{v} \frac{\frac{\tau}{Q} s}{\tau^{2} s^{2}+\frac{\tau}{Q} s+1}
$$

For the OTA-based $\mathrm{C}^{4}$

$$
\begin{aligned}
\tau^{2} & =\frac{C_{O} C_{T}-C_{2}^{2}}{G_{m 1} G_{m 2}} \\
\frac{\tau}{Q} & =\frac{C_{L}}{G_{m 1}}+\frac{C_{2}}{G_{m 2}}
\end{aligned}
$$




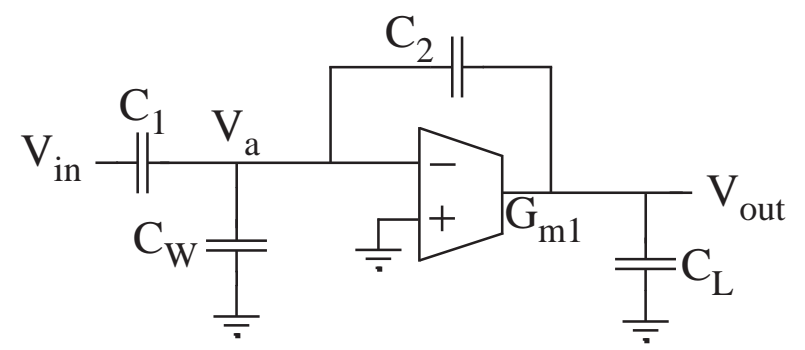

Figure B.2: Schematic of the OTA-C ${ }^{4}$ for transfer-function derivation at high frequencies.

Solving for $Q$ produces

$$
\begin{aligned}
Q & =\frac{\tau}{\frac{C_{L}}{G_{m 1}}+\frac{C_{2}}{G_{m 2}}} \\
& =\sqrt{\frac{C_{O} C_{T}-C_{2}^{2}}{G_{m 1} G_{m 2}}} \frac{G_{m 1} G_{m 2}}{G_{m 2} C_{L}+G_{m 1} C_{2}} \\
& =\frac{\sqrt{C_{O} C_{T}-C_{2}{ }^{2}} \sqrt{G_{m 1} G_{m 2}}}{G_{m 2} C_{L}+G_{m 1} C_{2}} \\
& =\frac{\sqrt{C_{O} C_{T}-C_{2}^{2}}}{C_{L} \sqrt{\frac{G_{m 2}}{G_{m 1}}}+C_{2} \sqrt{\frac{G_{m 1}}{G_{m 2}}}}
\end{aligned}
$$

And the passband gain $A_{v}$ is

$$
\begin{aligned}
\text { numerator } & =-s \frac{C_{1}}{G_{m 2}}\left(1-s \frac{C_{2}}{G_{m 2}}\right) \\
A_{v} \frac{\tau}{Q} & =-\frac{C_{1}}{G_{m 2}} \\
A_{v} & =-\frac{C_{1}}{G_{m 2}} \frac{Q}{\tau} \\
& =-\frac{C_{1}}{G_{m 2}} \frac{G_{m 1} G_{m 2}}{G_{m 2} C_{L}+G_{m 1} C_{2}} \\
& =-\frac{C_{1}}{C_{2}} \frac{G_{m 1}}{G_{m 2} \frac{C_{L}}{C_{2}}+G_{m 1}} \\
& =-\frac{C_{1}}{C_{2}} \frac{1}{1+\frac{C_{L}}{C_{2}} \frac{G_{m 2}}{G_{m 1}}}
\end{aligned}
$$


Brandon D. Rumberg

\section{B.1.2 $\mathrm{C}^{4}$ at High Frequencies}

Now let's consider the $\mathrm{C}^{4}$ at high frequencies. At node $V_{\text {out }}$

$$
\begin{aligned}
s C_{L} V_{\text {out }}+s C_{2}\left(V_{\text {out }}-V_{a}\right) & =-G_{m 1} V_{a} \\
s C_{O} V_{\text {out }} & =V_{a}\left(s C_{2}-G_{m 1}\right)
\end{aligned}
$$

and at node $V_{a}$

$$
\begin{gathered}
s C_{1}\left(V_{a}-V_{\text {in }}\right)+s C_{W} V_{a}+s C_{2}\left(V_{a}-V_{\text {out }}\right)=0 \\
V_{a} C_{T}=C_{1} V_{\text {in }}+C_{2} V_{\text {out }}
\end{gathered}
$$

Combining gives

$$
\begin{aligned}
\frac{s C_{O} V_{\text {out }}}{s C_{2}-G_{m 1}} C_{T} & =C_{1} V_{\text {in }}+C_{2} V_{\text {out }} \\
V_{\text {out }}\left(\frac{s C_{O} C_{T}-C_{2}\left(s C_{2}-G_{m 1}\right)}{s C_{2}-G_{m 1}}\right) & =C_{1} V_{\text {in }} \\
\frac{V_{\text {out }}}{V_{\text {in }}} & =C_{1} \frac{s C_{2}-G_{m 1}}{s\left(C_{O} C_{T}-C_{2}^{2}\right)+C_{2} G_{m 1}} \\
& =-\frac{C_{1}}{C_{2}} \frac{1-s \frac{C_{2}}{G_{m 1}}}{1+s \frac{C_{O} C_{T}-C_{2}{ }^{2}}{C_{2} G_{m 1}}} \\
& =-\frac{C_{1}}{C_{2}} \frac{1-s \tau_{f}}{1+s \tau_{h}}
\end{aligned}
$$

where

$$
\begin{aligned}
\tau_{f} & =\frac{C_{2}}{G_{m 1}} \\
\tau_{h} & =\frac{C_{O} C_{T}-C_{2}^{2}}{C_{2} G_{m 1}}
\end{aligned}
$$




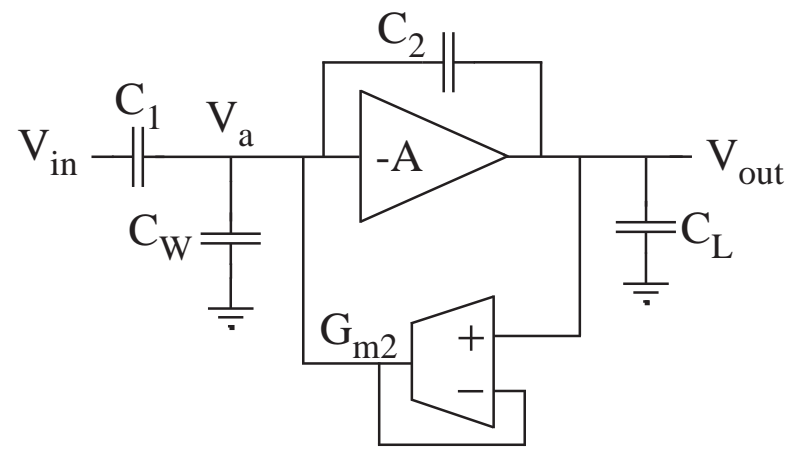

Figure B.3: Schematic of the OTA-C ${ }^{4}$ for transfer-function derivation at low frequencies.

\section{B.1.3 $\mathrm{C}^{4}$ at Low Frequencies}

Now let's consider the $\mathrm{C}^{4}$ at low frequencies. At node $V_{a}$

$$
\begin{aligned}
s C_{1} V_{\text {in }}+s C_{2} V_{\text {out }} & =-G_{m 2} V_{\text {out }} \\
V_{\text {out }}\left(\frac{s C_{2}}{G_{m 2}}+1\right) & =-s \frac{C_{1}}{G_{m 2}} V_{\text {in }} \\
\frac{V_{\text {out }}}{V_{\text {in }}} & =-\frac{s \frac{C_{1}}{G_{m 2}}}{1+s \frac{C_{2}}{G_{m 2}}} \\
& =-\frac{C_{1}}{C_{2}} \frac{s \frac{C_{2}}{G_{m 2}}}{1+s \frac{C_{2}}{G_{m 2}}} \\
& =-\frac{C_{1}}{C_{2}} \frac{s \tau_{l}}{1+s \tau_{l}}
\end{aligned}
$$

where

$$
\tau_{l}=\frac{C_{2}}{G_{m 2}}
$$

\section{B.1.4 Capacitive Feedthrough}

During normal operation we want to keep the corner caused by the capacitive feedthrough time-constant $\tau_{f}$ at a much higher frequency than the low-pass corner caused by $\tau_{h}$.

$$
\begin{aligned}
\tau_{f} & \ll \tau_{h} \\
\frac{C_{2}}{G_{m 1}} & \ll \frac{C_{O} C_{T}-C_{2}{ }^{2}}{C_{2} G_{m 1}} \\
2 C_{2}^{2} & \ll C_{O} C_{T} \\
2 C_{2}{ }^{2} & \ll\left(C_{2}+C_{L}\right)\left(C_{1}+C_{2}+C_{W}\right)
\end{aligned}
$$


If all capacitors are equal

$$
2 C^{2} \ll 6 C^{2}
$$

\section{B.1.5 Solving for $Q_{\max }$}

Now let's find the maximum $Q$ value.

$$
\begin{aligned}
Q & =\frac{\sqrt{C_{T} C_{O}-C_{2}{ }^{2}}}{C_{L} \sqrt{\frac{G_{m 2}}{G_{m 1}}}+C_{2} \sqrt{\frac{G_{m 1}}{G_{m 2}}}} \frac{\sqrt{\frac{G_{m 2}}{G_{m 1}}}}{\sqrt{\frac{G_{m 2}}{G_{m 1}}}} \\
& =\sqrt{\frac{G_{m 2}}{G_{m 1}}} \frac{\sqrt{C_{T} C_{O}-C_{2}{ }^{2}}}{C_{L} \frac{1}{G_{m 2}}+C_{2}} \frac{\frac{1}{C_{2}}}{\frac{1}{C_{2}}} \\
& =\sqrt{\frac{G_{m 2}}{G_{m 1}}} \sqrt{\frac{C_{T} C_{O}-C_{2}{ }^{2}}{C_{2}{ }^{2}}} \frac{1}{\frac{C_{L}}{C_{2}} \frac{G_{m 2}}{G_{m 1}}+1}
\end{aligned}
$$

Let

$$
\begin{aligned}
b & =\frac{G_{m 2}}{G_{m 1}} \\
a & =\frac{C_{L}}{C_{2}} \\
d & =\sqrt{\frac{C_{T} C_{O}-C_{2}^{2}}{C_{2}^{2}}} \\
Q & =\sqrt{b} d \frac{1}{a b+1} \\
& =\frac{\sqrt{b} d}{1+a b} \\
& =d b^{1 / 2}(1+a b)^{-1}
\end{aligned}
$$

Take the derivative

$$
\begin{aligned}
\frac{d Q}{d b} & =\frac{1}{2} d b^{-1 / 2}(1+a b)^{-1}+d b^{1 / 2}\left(-a(1+a b)^{-2}\right) \\
& =\frac{\frac{1}{2} d}{\sqrt{b}} \frac{1-a b}{(1+a b)^{2}}
\end{aligned}
$$

This equals zero when $1-a b=0$, which is when $b=\frac{1}{a}$ meaning $Q_{\max }$ occurs when

$$
\frac{C_{2}}{C_{L}}=\frac{G_{m 2}}{G_{m 1}}
$$




$$
\begin{aligned}
Q_{\max } & =\sqrt{\frac{1}{a}} \frac{d}{1+a \frac{1}{a}} \\
& =\frac{d}{2 \sqrt{a}} \\
& =\frac{1}{2} \sqrt{\frac{C_{T} C_{O}-C_{2}{ }^{2}}{C_{L} C_{2}}}
\end{aligned}
$$

The gain at $Q_{\max }$ is

$$
\begin{aligned}
A_{v} & =-\frac{C_{1}}{C_{2}} \frac{1}{1+a \frac{1}{a}} \\
& =-\frac{C_{1}}{2 C_{2}} \\
Q_{\max } & =\frac{1}{2} \sqrt{\frac{C_{T} C_{O}-C_{2}{ }^{2}}{C_{L} C_{2}}} \\
\left(2 Q_{\max }\right)^{2} & =\frac{C_{T} C_{O}-C_{2}^{2}}{C_{L} C_{2}}
\end{aligned}
$$


Let $X=\left(2 Q_{\max }\right)^{2}$

$$
\begin{aligned}
& X C_{2} C_{L}=C_{T} C_{O}-C_{2}^{2} \\
& C_{T} C_{O}=C_{2}\left(X C_{L}+C_{2}\right) \\
& =C_{2}\left(X C_{L}+X C_{2}-X C_{2}+C_{2}\right) \\
& =X C_{2} C_{O}+C_{2}^{2}(1-X) \\
& C_{T}=X C_{2}+\frac{C_{2}^{2}(1-X)}{C_{O}} \\
& =C_{2}\left(\frac{X\left(C_{2}+C_{L}\right)+C_{2}-C_{2} X}{C_{O}}\right) \\
& =C_{2}\left(\frac{X C_{L}+C_{2}}{C_{O}}\right) \\
& =C_{2}\left(1+\frac{C_{L}(X-1)}{C_{2}+C_{L}}\right) \\
& C_{1}+C_{W}=C_{2}\left(\frac{C_{L}(X-1)}{C_{2}+C_{L}}-\right) \\
& =C_{2}\left(\frac{\left(C_{L}+C_{2}-C_{2}\right)(X-1)}{C_{2}+C_{L}}\right) \\
& =C_{2} X-C_{2}-\frac{C_{2}^{2}(X-1)}{C_{2}+C_{L}} \\
& =C_{2}\left(X-1+\frac{C_{2}(1-X)}{C_{2}+C_{L}}\right) \\
& =C_{2}\left(\frac{C_{L}(X-1)}{C_{2}+C_{L}}\right) \\
& =(X-1) \frac{C_{2} C_{L}}{C_{2}+C_{L}} \\
& =(X-1) C_{2} \| C_{L} \\
& =\left(4 Q_{\max }^{2}-1\right) C_{2} \| C_{L}
\end{aligned}
$$

\section{B.2 OTA-C ${ }^{4}$ Noise Analysis}

The OTA's are the noise sources in an OTA-C ${ }^{4}$. We can determine the noise contribution of each OTA by placing a noise source at the non-inverting terminal of that OTA, grounding the input to the $\mathrm{C}^{4}$ and calculating the response at the output. Let's start with a noise source at the non-inverting terminal of the feed-forward OTA. 


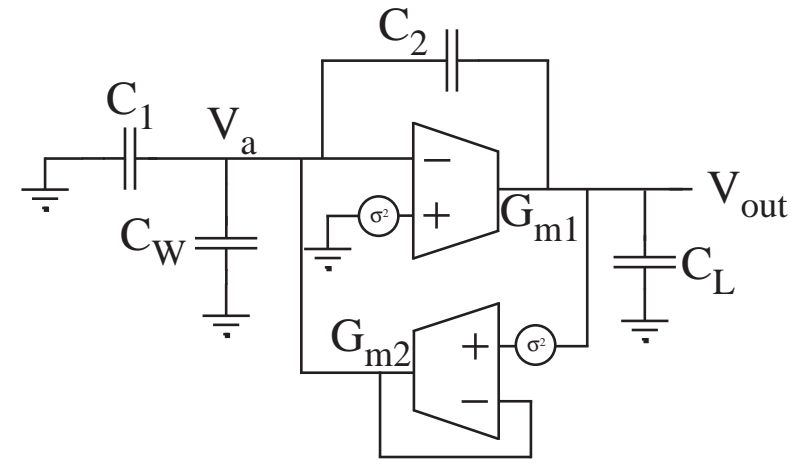

Figure B.4: Schematic of the OTA-C ${ }^{4}$ for noise analysis.

\section{B.2.1 Noise Transfer Function for $G_{m 1}$ Noise Source}

At node $V_{\text {out }}$ we have

$$
\begin{aligned}
s C_{L} V_{\text {out }}+s C_{2}\left(V_{\text {out }}-V_{a}\right) & =G_{m 1}\left(v_{1 n}-V_{a}\right) \\
s C_{O} V_{\text {out }} & =G_{m 1} v_{1 n}+V_{a}\left(s C_{2}-G_{m 1}\right)
\end{aligned}
$$

and at node $V_{a}$ we have

$$
\begin{aligned}
s\left(C_{1}+C_{W}\right) V_{a}+s C_{2}\left(V_{a}-V_{\text {out }}\right) & =G_{m 2}\left(V_{\text {out }}-V_{a}\right) \\
V_{a}\left(s C_{t}+G_{m 2}\right) & =V_{\text {out }}\left(G_{m 2}+s C_{2}\right)
\end{aligned}
$$

Combining equations B.77 and B.79 we have

$$
\begin{aligned}
s C_{O} V_{\text {out }}= & G_{m 1} v_{\text {in }}+\left(s C_{2}-G_{m 1}\right) V_{\text {out }} \frac{G_{m 2}+s C_{2}}{s C_{T}+G_{m 2}} \\
V_{\text {out }}\left(s^{2} C_{O} C_{T}+s C_{O} G_{m 2}\right)= & v_{\text {in }}\left(s G_{m 1} C_{T}+G_{m 1} G_{m 2}\right) \\
& +V_{\text {out }}\left(s C_{2}-G_{m 1}\right)\left(G_{m 2}+s C_{2}\right) \\
V_{\text {out }}= & v_{\text {in }} \frac{s \frac{C_{T}}{G_{m 2}}+1}{s^{2} \frac{C_{O} C_{T}-C_{2}{ }^{2}}{G_{m 1} G_{m 2}}+s\left(\frac{C_{L}}{G_{m 1}}+\frac{C_{2}}{G_{m 2}+1}\right)+1}
\end{aligned}
$$

Note that

$$
\frac{C_{T}}{G_{m 2}}=\frac{C_{T}}{C_{1}} \tau_{l}
$$

so this is effectively a high-pass function of noise. 
Brandon D. Rumberg

Appendix

\section{B.2.2 Noise Transfer Function for $G_{m 2}$ Noise Source}

Next we will look at the noise contribution for the feed-back OTA. Starting with node $V_{\text {out }}$

$$
\begin{aligned}
s C_{L} V_{\text {out }}+s C_{2}\left(V_{\text {out }}-V_{a}\right) & =-G_{m 1} V_{a} \\
s C_{O} V_{\text {out }} & =V_{a}\left(s C_{2}-G_{m 1}\right)
\end{aligned}
$$

and at node $V_{a}$

$$
\begin{aligned}
G_{m 2}\left(V_{\text {out }}-V_{a}\right)+i_{2 n} & =s\left(C_{1}+C_{W}\right) V_{a}+s C_{2}\left(V_{a}-V_{\text {out }}\right) \\
V_{\text {out }}\left(s C_{2}+G_{m 2}\right)+i_{2 n} & =\left(s C_{T}+G_{m 2}\right) V_{a}
\end{aligned}
$$

Combining these equations we get

$$
\begin{aligned}
\left(s C_{T}+G_{m 2}\right) s C_{O} V_{\text {out }}= & V_{\text {out }}\left(s^{2} C_{2}^{2}+s C_{2}\left(G_{m 2}-G_{m 1}\right)-G_{m 1} G_{m 2}\right) \\
& +i_{2 n}\left(s C_{2}-G_{m 1}\right) \\
V_{\text {out }}= & v_{2 n} \frac{s \frac{C_{2}}{G_{m 1}}-1}{s^{2} \frac{C_{T} C_{O}-C_{2}{ }^{2}}{G_{m 1} G_{m 2}}+s\left(\frac{C_{L}}{G_{m 1}}+\frac{C_{2}}{G_{m 2}}\right)+1}
\end{aligned}
$$

Since $\frac{C_{2}}{G_{m 1}}=\tau_{f}$, and $\tau_{f}$ is far outside the passband, we can approximate

$$
V_{\text {out }} \approx v_{2 n} \frac{1}{s^{2} \frac{C_{T} C_{O}-C_{2}^{2}}{G_{m 1} G_{m 2}}+s\left(\frac{C_{L}}{G_{m 1}}+\frac{C_{2}}{G_{m 2}}\right)+1}
$$

\section{B.2.3 Integrated Noise}

Now to combine and integrate the noise. The standard form for the equivalent noise bandwidth of a bandpass filter is

$$
\int_{0}^{\infty} \frac{\left(\frac{f}{f_{0} Q}\right)^{2}}{\left(1-\left(\frac{f}{f_{0}}\right)^{2}\right)^{2}+\left(\frac{f}{f_{0} Q}\right)^{2}} d f=\frac{\pi}{2} \frac{f_{0}}{Q}
$$

also

$$
\int_{0}^{\infty} \frac{1}{\left(1-\left(\frac{f}{f_{0}}\right)^{2}\right)^{2}+\left(\frac{f}{f_{0} Q}\right)^{2}} d f=\frac{\pi}{2} f_{0} Q
$$


where

$$
\begin{aligned}
f_{0} & =\frac{1}{2 \pi \tau} \\
& =\frac{1}{2 \pi} \sqrt{\frac{G_{m 1} G_{m 2}}{C_{O} C_{T}-C_{2}{ }^{2}}} \\
Q & =\frac{\sqrt{C_{O} C_{T}-C_{2}{ }^{2}}}{C_{L} \sqrt{\frac{G_{m 2}}{G_{m 1}}}+C_{2} \sqrt{\frac{G_{m 1}}{G_{m 2}}}}
\end{aligned}
$$

The full noise of the circuit is

$$
\begin{aligned}
\hat{V}_{\text {out }}^{2}= & \int_{0}^{\infty} \frac{v_{1 n}{ }^{2}}{\left(1-\left(\frac{f}{f_{0}}\right)^{2}\right)^{2}+\left(\frac{f}{f_{0} Q}\right)^{2}} d f \\
& +\int_{0}^{\infty} \frac{v_{1 n}{ }^{2} A_{v 1}{ }^{2}\left(\frac{f}{f_{0} Q}\right)^{2}}{\left(1-\left(\frac{f}{f_{0}}\right)^{2}\right)^{2}+\left(\frac{f}{f_{0} Q}\right)^{2}} d f \\
& +\int_{0}^{\infty} \frac{v_{2 n}{ }^{2}}{\left(1-\left(\frac{f}{f_{0}}\right)^{2}\right)^{2}+\left(\frac{f}{f_{0} Q}\right)^{2}} d f \\
& +\int_{0}^{\infty} \frac{v_{2 n}{ }^{2} A_{v 2}{ }^{2}\left(\frac{f}{f_{0} Q}\right)^{2}}{\left(1-\left(\frac{f}{f_{0}}\right)^{2}\right)^{2}+\left(\frac{f}{f_{0} Q}\right)^{2}} d f
\end{aligned}
$$

where

$$
\begin{aligned}
A_{v 1} \frac{\tau}{Q} & =\frac{C_{T}}{G_{m 2}} \\
A_{v 1} & =\frac{C_{T}}{G_{m 2}} \frac{Q}{\tau} \\
& =\frac{C_{T}}{G_{m 2}} \frac{G_{m 1} G_{m 2}}{G_{m 2} C_{L}+G_{m 1} C_{2}} \\
& =\frac{G_{m 1} C_{T}}{G_{m 2} C_{L}+G_{m 1} C_{2}} \\
A_{v 2} \frac{\tau}{Q} & =\frac{C_{2}}{G_{m 1}} \\
A_{v 2} & =\frac{C_{2}}{G_{m 1}} \frac{Q}{\tau} \\
& =\frac{C_{2}}{G_{m 1}} \frac{G_{m 1} G_{m 2}}{G_{m 2} C_{L}+G_{m 1} C_{2}} \\
& =\frac{G_{m 2} C_{2}}{G_{m 2} C_{L}+G_{m 1} C_{2}}
\end{aligned}
$$


Brandon D. Rumberg

$$
\begin{aligned}
f_{0} Q & =\frac{1}{2 \pi} \sqrt{\frac{G_{m 1} G_{m 2}}{C_{O} C_{T}-C_{2}^{2}}} \frac{\sqrt{C_{O} C_{T}-C_{2}^{2}}}{C_{L} \sqrt{\frac{G_{m 2}}{G_{m 1}}}+C_{2} \sqrt{\frac{G_{m 1}}{G_{m 2}}}} \\
& =\frac{1}{2 \pi} \frac{G_{m 1} G_{m 2}}{C_{L} G_{m 2}+C_{2} G_{m 1}} \\
\frac{f_{0}}{Q} & =\frac{1}{2 \pi} \sqrt{\frac{G_{m 1} G_{m 2}}{C_{O} C_{T}-C_{2}{ }^{2}}} \frac{C_{L} \sqrt{\frac{G_{m 2}}{G_{m 1}}}+C_{2} \sqrt{\frac{G_{m 1}}{G_{m 2}}}}{\sqrt{C_{O} C_{T}-C_{2}^{2}}} \\
& =\frac{1}{2 \pi} \frac{C_{L} G_{m 2}+C_{2} G_{m 1}}{C_{O} C_{T}-C_{2}{ }^{2}}
\end{aligned}
$$

Using the integral results listed above

$$
\begin{aligned}
\hat{V}_{\text {out }}^{2}= & v_{1 n}^{2} \frac{\pi}{2} f_{0} Q+v_{1 n}^{2} A_{v 1}^{2} \frac{\pi}{2} \frac{f_{0}}{Q}+v_{2 n}^{2} \frac{\pi}{2} f_{0} Q+v_{2 n}^{2} A_{v 2}^{2} \frac{\pi}{2} \frac{f_{0}}{Q} \\
= & v_{1 n}^{2} \frac{\pi}{2} \frac{1}{2 \pi} \frac{G_{m 1} G_{m 2}}{C_{L} G_{m 2}+C_{2} G_{m 1}} \\
& +v_{1 n}^{2} \frac{\pi}{2}\left(\frac{G_{m 1} C_{T}}{G_{m 2} C_{L}+G_{m 1} C_{2}}\right)^{2} \frac{1}{2 \pi} \frac{C_{L} G_{m 2}+C_{2} G_{m 1}}{C_{O} C_{T}-C_{2}^{2}} \\
& +v_{2 n}^{2} \frac{\pi}{2} \frac{1}{2 \pi} \frac{G_{m 1} G_{m 2}}{C_{L} G_{m 2}+C_{2} G_{m 1}} \\
& +v_{2 n}^{2} \frac{\pi}{2}\left(\frac{G_{m 2} C_{2}}{G_{m 2} C_{L}+G_{m 1} C_{2}}\right)^{2} \frac{1}{2 \pi} \frac{C_{L} G_{m 2}+C_{2} G_{m 1}}{C_{O} C_{T}-C_{2}^{2}} \\
\hat{V}_{\text {out }}^{2}= & \frac{v_{1 n}^{2}}{4} \frac{G_{m 1} G_{m 2}}{C_{L} G_{m 2}+C_{2} G_{m 1}}+\frac{v_{1 n}^{2}}{4} \frac{C_{T}^{2}}{C_{O} C_{T}-C_{2}^{2}} \frac{G_{m 1}^{2}}{G_{m 2} C_{L}+G_{m 1} C_{2}} \\
+ & \frac{v_{2 n}^{2}}{4} \frac{G_{m 1} G_{m 2}}{C_{L} G_{m 2}+C_{2} G_{m 1}}+\frac{v_{2 n}^{2}}{4} \frac{C_{2}^{2}}{C_{O} C_{T}-C_{2}^{2}} \frac{G_{m 2}^{2}}{G_{m 2} C_{L}+G_{m 1} C_{2}}
\end{aligned}
$$

When $Q=Q_{\max }, G_{m 1}=G_{m 2} \frac{C_{L}}{C_{2}}$ and $G_{m 2}=G_{m 1} \frac{C_{2}}{C_{L}}$. This simplifies the above expression to

$$
\hat{V}_{\text {out }}^{2}=\frac{v_{1 n}^{2}}{4} \frac{G_{m 1}}{2 C_{L}}+\frac{v_{1 n}^{2}}{4} \frac{C_{T}^{2}}{C_{O} C_{T}-C_{2}^{2}} \frac{G_{m 1}}{2 C_{2}}+\frac{v_{2 n}^{2}}{4} \frac{G_{m 2}}{2 C_{2}}+\frac{v_{2 n}^{2}}{4} \frac{C_{2}^{2}}{C_{O} C_{T}-C_{2}^{2}} \frac{G_{m 2}}{2 C_{L}}
$$

Assuming that $C_{T}^{2} \gg C_{2}^{2}$, this can be approximated as

$$
\hat{V}_{\text {out }}^{2}=\frac{v_{1 n}^{2}}{4} \frac{G_{m 1}}{2 C_{L}}+\frac{v_{1 n}^{2}}{4} \frac{C_{T} G_{m 1}}{2 C_{2} C_{O}}+\frac{v_{2 n}^{2}}{4} \frac{G_{m 2}}{2 C_{2}}+\frac{v_{2 n}^{2}}{4} \frac{G_{m 2} C_{2}^{2}}{2 C_{T} C_{L}^{2}}
$$

The last term makes contributes very little in relation to the rest of the terms so it can be neglected. 
Brandon D. Rumberg

For an OTA the noise is

$$
v_{n}^{2}=\frac{N q V_{L}^{2}}{I_{b}}=\frac{N q I_{b}}{G_{m}^{2}}=\frac{N q V_{L}}{G_{m}}
$$

where $N$ is the number of noise sources. We will assume that both OTA's have the same number of noise sources and linear range $V_{L}$.

$$
\hat{V}_{\text {out }}^{2}=\frac{N q V_{L}}{8 C_{L}}+\frac{N q V_{L} C_{T}}{8 C_{2} C_{O}}+\frac{N q V_{L}}{8 C_{2}}
$$

Under normal operation, $C_{L}$ is very large so the first term can be neglected.

$$
\begin{gathered}
\hat{V}_{\text {out }}^{2}=\frac{N q V_{L}}{8 C_{2}}\left(\frac{C_{T}}{C_{O}}+1\right) \\
Q_{\text {max }}^{2}=\frac{1}{4} \frac{C_{T} C_{O}-C_{2}^{2}}{C_{L} C_{2}} \approx \frac{1}{4} \frac{C_{T} C_{O}}{C_{L} C_{2}} \approx \frac{1}{4} \frac{C_{T}}{C_{2}}
\end{gathered}
$$

There are three cases to consider.

Case 1: $\frac{C_{T}}{C_{O}} \gg 1$

$$
\begin{aligned}
\hat{V}_{\text {out }}^{2} & \approx \frac{N q V_{L} C_{T}}{8 C_{2} C_{O}} \approx \frac{N q V_{L} Q_{\max }^{2}}{2 C_{O}} \\
S N R & =10 \log _{10}\left(\frac{\left(\frac{V_{L}}{\sqrt{2}}\right)^{2}}{\hat{V}_{\text {out }}^{2}}\right)=10 \log _{10}\left(\frac{V_{L} C_{O}}{N q Q_{\text {max }}^{2}}\right)
\end{aligned}
$$

Case 2: $\frac{C_{T}}{C_{O}}=1$

$$
\begin{aligned}
\hat{V}_{\text {out }}^{2} & \approx \frac{N q V_{L}}{4 C_{2}} \\
S N R & =10 \log _{10}\left(\frac{2 V_{L} C_{2}}{N q}\right)
\end{aligned}
$$

Case 3: $\frac{C_{T}}{C_{O}} \ll 1$

$$
\begin{aligned}
\hat{V}_{\text {out }}^{2} & \approx \frac{N q V_{L}}{8 C_{2}} \approx \frac{N q V_{L} Q_{\max }^{2}}{2 C_{T}} \\
S N R & =10 \log _{10}\left(\frac{V_{L} C_{T}}{N q Q_{\max }^{2}}\right)
\end{aligned}
$$


Brandon D. Rumberg

\section{B.3 Power Analysis}

In this section we will derive useful expressions for the power consumed by the circuit.

$$
\text { Power }=V_{d d} I_{d r a w}=V_{d d}\left(I_{B 1}+I B 2\right)=V_{d d}\left(V_{L 1} G_{m 1}+V_{L 2} G_{m 2}\right)
$$

For standard OTA's $I_{B}=\frac{2 U_{T} G_{m}}{\kappa}$ so

$$
\text { Power }=V_{d d}\left(\frac{2 U_{T} G_{m 1}}{\kappa}+\frac{2 U_{T} G_{m 2}}{\kappa}\right)
$$

When operating at $Q_{\max }$ we have $\frac{G_{m 1}}{G_{m 2}}=\frac{C_{L}}{C_{2}}$ so

$$
\begin{aligned}
\text { Power } & =V_{d d} G_{m 1}\left(V_{L 1}+V_{L 2} \frac{C_{2}}{C_{L}}\right) \\
\frac{2 C_{L} Q_{m a x}}{\tau} & =2 C_{L}\left(\frac{1}{2} \sqrt{\frac{C_{T} C_{O}-C_{2}^{2}}{C_{L} C_{2}}}\right)\left(\sqrt{\frac{G_{m 1} G_{m 2}}{C_{O} C_{T}-C_{2}^{2}}}\right) \\
& \approx 2 C_{L}\left(\frac{1}{2} \sqrt{\frac{C_{T}}{C_{2}}}\right)\left(\sqrt{\frac{G_{m 1}^{2} \frac{C_{2}}{C_{L}}}{C_{T} C_{O}}}\right) \\
& \approx \frac{C_{L}}{\sqrt{C_{O} C_{L}}} G_{m 1} \\
& \approx G_{m 1}
\end{aligned}
$$

Using this result we can write the power as

$$
\begin{aligned}
\text { Power } & =V_{d d}\left(V_{L 1}+V_{L 2} \frac{C_{2}}{C_{L}}\right) \frac{2 C_{L} Q_{\max }}{\tau} \\
& =V_{d d}\left(C_{L} V_{L 1}+C_{2} V_{L 2}\right) 4 \pi Q_{\max } f_{\text {center }} \\
& =4 \pi C_{L} V_{L 1} V_{d d} Q_{\max } f_{\text {center }} \\
& =4 \pi C_{L} V_{L 1} V_{d d} Q_{\max } \frac{G_{\operatorname{mi} 1}}{4 \pi Q_{\max } C_{L}}
\end{aligned}
$$

\section{B.4 Design Procedure}

Here we present a method for designing an OTA-C $\mathrm{C}^{4}$ given the following specifications.

- $Q$ 
- $S N R$

- $f_{\text {center }}$

- $V_{i n, \max }$

1. Design $C_{L}$ sufficiently such that $C_{L} \gg C_{T}, C_{T}$ will be determined later.

$$
\hat{V}_{\text {out }}^{2} \approx \frac{N q V_{L}}{8 C_{2}}
$$

2. Choose $C_{2}$ to meet given SNR specs. This is assuming $V_{\text {out }}=V_{L}$.

$$
\begin{aligned}
S N R & =10 \log _{10}\left(\frac{4 V_{L} C_{2}}{N q}\right) \\
C_{2} & =\frac{N q}{4 V_{L}} 10^{\frac{S N R}{10}}
\end{aligned}
$$

3. If the gain is given, choose $C_{1}$.

$$
\begin{aligned}
& A_{v}=\frac{-C_{1}}{2 C_{2}} \\
& C_{1}=2 C_{2} A_{v}
\end{aligned}
$$

4. Choose $C_{T}$ for linearity.

$$
\begin{aligned}
V_{\text {out }, \text { max }} & =A_{v} V_{\text {in, } \text { max }}=\frac{C_{1}}{2 C_{2}} V_{\text {in, } \text { max }}=\frac{C_{T}}{2 C_{2}} V_{L} \\
& \approx 2 Q_{\text {max }}^{2} V_{L} \\
C_{T} & \geq \frac{C_{1} V_{i n, \max }}{V_{L}} \\
C_{T} & =4 C_{2} Q_{\text {max }}
\end{aligned}
$$

5. If $A_{v}$ is not given.

$$
\begin{aligned}
V_{i n, \max } & \approx \frac{2 C_{2}}{C_{1}} V_{L} \\
C_{1} & =\frac{2 C_{2} V_{L}}{V_{i n, \max }}
\end{aligned}
$$

6.

$$
\begin{gathered}
4 \pi Q_{\text {max }} C_{2} f_{\text {center }}=4 \pi \frac{1}{2} \sqrt{C_{T}} C_{2} C_{2} \frac{1}{2 \pi} \sqrt{\frac{G_{m 2}^{2} \frac{C_{L}}{C_{2}}}{C_{T} C_{O}}}=G_{m 2} \\
G_{m 2}=4 \pi Q_{\text {max }} C_{2} f_{\text {center }}
\end{gathered}
$$


7.

$$
G_{m 1}=G_{m 2} \frac{C_{2}}{C_{L}}
$$

8. $C_{L}$ must be $\gg C_{T}$; otherwise it can be optimized for power.

$$
P=\frac{C_{L}}{V_{L}} V_{d d} Q_{\text {max }} f_{\text {center }}
$$




\section{References}

[1] S. Liu, J. Kramer, G. Indiveri, T. Delbruck, and R. Douglas, Analog VLSI: Circuits and Principles, The MIT Press, 2002.

[2] I. Greenwood Jr., J. Holdam Jr., and D. Machrae Jr., Electronic Instruments, volume 21 of MIT Radiation Laboratory Series, McGraw-Hill, 1948.

[3] C. Mead, Analog VLSI and Neural Systems, Addison-Wesley, 1989.

[4] C. Enz, F. Krummanacher, and E. Vittoz, "An analytical MOS transistor model valid in all regions of operation and dedicated to low-voltage and low-current applications," Journal on Analog Integrated Circuits and Signal Processsing, , no. 1, pp. 83-114, July 1995.

[5] G. Serrano, P. Smith, H. Lo, R. Chawla, T. Hall, C. Twigg, and P. Hasler, "Automatic rapid programming of large arrays of floating-gate elements," in Proceedings of the IEEE International Symposium on Circuits and Systems, Vancouver, BC, Canada, May 2004, vol. 1, pp. 1373-1376.

[6] D. Graham, E. Farquhar, B. Degnan, C. Gordon, and P. Hasler, "Indirect programming of floating-gate transistors," vol. 54, no. 5, pp. 951-963, May 2007.

[7] F. Adil, G. Serrano, and P. Hasler, "Offset removal using floating-gate circuits for mixed-signal systems," in Proceedings of the IEEE Southwest Symposium on MixedSignal Design, February 2003, pp. 190-195.

[8] P. Hasler, B. Minch, and C. Diorio, "Adaptive circuits using pFET floating-gate devices," in Proceedings of the 20th Anniversary Conference on Advanced Research in VLSI, Atlanta, GA, March 1999, pp. 215-229.

[9] P. Hasler and J. Dugger, "An analog floating-gate node for supervised learning," IEEE Transactions on Circuits and Systems I, Regular Papers, vol. 52, no. 5, pp. 834-845, May 2005.

[10] P. Hasler, P. Smith, D. Graham, R. Ellis, and D. Anderson, "Analog floating-gate, onchip auditory sensing system interfaces," IEEE Sensors Journal, vol. 5, pp. 1027-1034, October 2005. 
[11] G. Efthivoulidis, L. Toth, and Y. Tsividis, "Noise in Gm-C filters," IEEE Transactions on Circuits and Systems - II, vol. 45, no. 3, 1998.

[12] D. Graham, P. Smith, R. Chawla, and P. Hasler, "A programmable bandpass array using floating-gate elements," in Proceedings of the IEEE International Symposium on Circuits and Systems, Vancouver, BC, Canada, May 2004, vol. 1, pp. I-97 I-100.

[13] P. Furth and H. Ommani, "Low-voltage highly-linear transconductor design in subthreshold CMOS," in Proceedings of the IEEE Midwest Symposium on Circuits and Systems, Sacramento, CA, August 1997, vol. 1, pp. 156-159.

[14] R. Sarpeshkar, R. Lyon, and C. Mead, "A low-power wide-linear-range transconductance amplifier," Analog Integrated Circuits and Signal Processing, vol. 13, no. 1-2, pp. $123-51,1997$.

[15] C. Kitchin and L. Counts, "RMS to DC conversion application guide," Electronic Product Design, vol. 5, no. 3, pp. 149 - 57, 1984.

[16] M. Steyaert, W. Dehaene, J. Craninckx, M. Walsh, and P. Real, "A CMOS rectifierintegrator for amplitude detection in hard disk servo loops," USA, 1995, vol. 30, pp. $743-51$.

[17] J. Mulder, A. Van der Woerd, W. Serdijn, and A. Van Roermund, "An RMS-DC converter based on the dynamic translinear principle," USA, 1997, vol. 32, pp. 1146 50 .

[18] B. Gilbert, "Novel technique for R.M.S.-D.C. conversion based on the difference of squares," Electronics Letters, vol. 11, no. 8, pp. 181 - 2, 1975.

[19] T. Delbruck, "Bump circuits for computing similarity and dissimilarity of analog voltages," Seattle, WA, USA, 1991, pp. 475 - 479.

[20] R. Lyon and C. Mead, "An analog electronic cochlea," IEEE Transactions on Acoustics, Speech, and Signal Processing, vol. 36, pp. 1119-1134, 1988.

[21] L. Watts, D. Kerns, R. Lyon, and C. Mead, "Improved implementation of the silicon cochlea," IEEE Journal of Solid-State Circuits, vol. 27, no. 5, pp. 692-700, May 1992.

[22] A. van Schaik, E. Fragnière, and E. Vittoz, "Improved silicon cochlea using compatible lateral bipolar transistors," in Advances in Neural Information Processing Systems 8, D. Touretzky, Ed., Cambridge, MA, 1996, pp. 671-677, MIT Press.

[23] R. Sarpeshkar, R. Lyon, and C. Mead, "An analog VLSI cochlea with new transconductance amplifiers and nonlinear gain control," in Proceedings of the IEEE International Symposium on Circuits and Systems, Atlanta, GA, 1996, vol. 3, pp. 292-296.

[24] D. Graham and P. Hasler, "Capacitively-coupled current conveyer second-order sections for continuous-time bandpass filtering and cochlea modeling," in Proceedings of the IEEE International Symposium on Circuits and Systems, Scottsdale, AZ, May 2002, vol. 5, pp. V-485-V-488. 
[25] B. Wen and K. Boahen, "Active bidirectional coupling in a cochlear chip," in $A d$ vances in Neural Information Processing Systems 9, B. Sholkopf and Y. Weiss, Eds., Cambridge, MA, 2006, MIT Press.

[26] T. Massengill, D. Wilson, P. Hasler, and D. Graham, "Empirical comparison of analog and digital auditory preprocessing for automatic speech recognition," in Proceedings of the IEEE International Symposium on Circuits and Systems, Scottsdale, AZ, May 2002, vol. 5, pp. 77-80.

[27] R. Ellis, H. Yoo, D. Graham, P. Hasler, and D. Anderson, "A continuous-time speech enhancement front-end for microphone inputs," in Proceedings of the IEEE International Symposium on Circuits and Systems, Scottsdale, AZ, May 2002, vol. 2, pp. II-728 - II-731.

[28] A. van Schaik and S. Shamma, "A neuromorphic sound localizer for a smart MEMS system," in Proceedings of the International Symposium on Circuits and Systems, May 2003, vol. 4, pp. IV-864 - IV-867.

[29] B. Rumberg, K. McMillan, C. Rea, and D. Graham, "Lateral coupling in silicon cochlear models," in Proceedings of the IEEE Midwest Symposium on Circuits and Systems, Knoxville, TN, August 2008, vol. 1, pp. 25-28.

[30] L. Watts, Cochlear Mechanics: Analysis and Analog VLSI, Ph.D. thesis, California Institute of Technology, Pasadena, CA, 1993.

[31] E. Kandel, J. Schwartz, and T. Jessel, Principles of Neural Science, McGraw-Hill, New York, 4th edition, 2000.

[32] T. Gold and R. Pumphrey, "Hearing. I. The cochlea as a frequency analyzer," Proceedings of the Royal Society of London. Series B, Biological Sciences, vol. 135, no. 881, pp. 462-491, December 1948.

[33] C. Salthouse and R. Sarpeshkar, "A practical micropower programmable bandpass filter for use in bionic ears," IEEE Journal of Solid State Circuits, vol. 38, no. 1, pp. 63-70, January 2003.

[34] A. van Schaik and E. Fragnière, "Pseudo-voltage domain implementation of a 2dimensional silicon cochlea," in Proceedings of the IEEE International Symposium on Circuits and Systems, Sydney, NSW, Australia, May 2001, vol. 2, pp. 185-188.

[35] E. Fragnière, "A 100-channel analog CMOS auditory filter bank for speech recognition," in Solid-State Circuits Conference, 2005. Digest of Technical Papers. ISSCC. 2005 IEEE International, San Francisco, CA, February 2005, vol. 1, pp. 140-589.

[36] L. Doherty, B. Warneke, B. Boser, and K. Pister, "Energy and performance considerations for smart dust," International Journal of Parallel and Distributed Systems and Networks, vol. 4, no. 3, pp. 121 - 133, 2001. 
[37] S. Jevtic, M. Kotowsky, R. Dick, P. Dindap, and C. Dowding, "Lucid dreaming: reliable analog event detection for energy-constrained applications," Piscataway, NJ, USA, 2007, pp. $350-359$.

[38] K. Chen and C. Svensson, "A/D conversion and analog vector quantization using neural network models," in First IEEE International Conference on Artifical Neural Networks, October 1989, pp. 324-328.

[39] S. Rovetta and R. Zunino, "Analog vector quantization chip for flexible video compression systems," in Proceedings of the IEEE International Symposium on Circuits and Systems, May 1999, vol. 4, pp. 367-370.

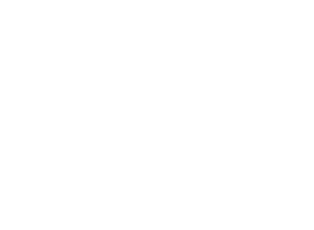

